\title{
ASYMPTOTIC EXPANSIONS FOR TOEPLITZ OPERATORS ON SYMMETRIC SPACES OF GENERAL TYPE
}

\author{
MIROSLAV ENGLIŠ AND HARALD UPMEIER
}

\begin{abstract}
A general theory of Berezin-Toeplitz quantization for symmetric spaces is presented, with emphasis on representation-theoretic asymptotic expansions, which applies to spaces of compact and non-compact type, both in the classical setting of hermitian symmetric spaces and also for their real forms. The Berezin (or Wick type) calculus and its opposite "anti-Wick" type calculus are treated on an equal footing.
\end{abstract}

\section{INTRODUCTION}

In this paper we give a comprehensive and uniform treatment of Toeplitz quantization and associated asymptotic expansions (for the Berezin transform and Moyal type star-products) in the general setting of symmetric spaces of compact or noncompact type, both in the real and the complex hermitian case. More precisely, the theory is developed for all symmetric spaces arising from real or complex Jordan algebras and Jordan triples. Thus the compact version is the so-called conformal compactification of a simple Jordan triple, and the non-compact version is the unit ball with respect to the spectral norm, which is a real or complex bounded symmetric domain. The flat case is also included.

The present paper can be regarded as a final step in the program started with our earlier papers [EU1, EU3] and [EU4, with the goal of developing a uniform theory for Toeplitz-Berezin quantization of symmetric spaces, which treats (i) compact and non-compact spaces, (ii) hermitian spaces and their real forms, and (iii) the calculus and its opposite "anti-calculus", on an equal footing. Such a uniform treatment reveals the underlying algebraic structure and will also be important for more advanced quantization methods such as the Weyl calculus. The first two papers EU1, EU3] dealt with the non-compact case where an important ingredient was a certain factorization property, which in some sense reduced the problem to obtaining the Peter-Weyl decomposition for the associated Berezin transform and its formal inverse. In the non-compact case, the existence of enough holomorphic functions makes the proof of the factorization property relatively easy. As a first step in the compact case, the paper [EU4] established the Peter-Weyl decomposition for the Berezin transform, but not for the other operations.

Received by the editors April 27, 2012 and, in revised form, March 9, 2013.

2010 Mathematics Subject Classification. Primary 32M15; Secondary 46E22, 47B35, 53D55.

Key words and phrases. Symmetric space, symmetric domain, Berezin quantization, Toeplitz quantization, Berezin transform.

The first author's research was supported by GAČR grant no. 201/09/0473 and AV ČR institutional research plan AV0Z10190503.

The second author's research was supported by GIF grant no. 696-17.6/2001. 
This left two major open problems in the compact case, namely (i) the proof of the factorization property, and (ii) a reciprocity formula for the asymptotic expansion of the Berezin transform and its inverse. The current paper establishes these results, together with (iii) the spectral decomposition of the Berezin transform in the real compact case. With these tools we are able to present a Peter-Weyl decomposition for the asymptotic expansions of the Berezin transform and the Wick type star-product, as well as the inverse Berezin transform and the anti-Wick type star-product in full generality. This also leads to a completely uniform exposition of the theory, thereby demonstrating the inherent algebraic relationships.

Our proof of the factorization properties based on a localization procedure, which allows us to treat the compact case, both in the real and complex situation, in a uniform fashion using the concept of Hartogs' domains, may be significant in more general situations, for example in studying Hardy type spaces on line bundles over compact complex manifolds.

In the paper EU2, the current authors obtained another kind of expansion, of geometric rather than representation-theoretic nature, in the non-compact case; the analogue of this expansion for the compact symmetric spaces remains to be explored. A further interesting development concerns non-holomorphic Bergman spaces associated with magnetic potentials.

However, the most important area of future study is the vector-valued case, i.e. the full holomorphic discrete series, as compared to the scalar-valued case treated in this paper. The vector-valued case corresponds to holomorphic induction of irreducible $K$-representations which are not characters, but can still be realized in a geometric way as holomorphic sections of line bundles over co-adjoint $K$-orbits $K(o)$ C]. In this way the classical phase space becomes $D \times K(o) \approx G(o)$, and the study of Berezin transforms and associated Wick type products in terms of $G$-invariant differential operators on $G(o)$ is quite promising. On the other hand, it is clear that a detailed representation-theoretic study will be combinatorially more difficult, involving higher multiplicities for the $K(o)$-orbits and matrix type Gamma-functions. These problems are under active investigation.

\section{SymmetriC SPACES AND JORDAN TRIPLES}

In this section we recall the Jordan theoretic description of symmetric spaces needed in the sequel. For more details, cf. [L], FK, EU1.

Let $Z$ be an irreducible hermitian Jordan triple of rank $r$ and dimension $d$. Denoting the Jordan triple product of $u, v, w \in Z$ by $\left\{u v^{*} w\right\}$, we put

$$
D(u, v) w:=2\left\{u v^{*} w\right\}, \quad Q_{z} w:=\left\{z w^{*} z\right\} .
$$

There are two ways to obtain irreducible real Jordan triples starting from an irreducible hermitian Jordan triple $Z$. In the real case let $z \mapsto z^{\sharp}$ be a conjugation of $Z$ preserving the Jordan triple product. The fixed points

$$
Z_{\mathbf{R}}:=\left\{x \in Z: x^{\sharp}=x\right\}
$$

form an irreducible real Jordan triple. In the complex case we consider the real Jordan triple $Z_{\mathbf{C}}$ underlying $Z$. As a rule we will use the subscript $\mathbf{K}=\mathbf{R}, \mathbf{C}$ for objects associated with the real form $Z_{\mathbf{K}}$. A maximal tripotent $e \in Z$ induces a Peirce decomposition $[\mathrm{L}$ ]

$$
Z=Z^{1} \oplus Z^{1 / 2}
$$


and $Z^{1}$ is an irreducible complex Jordan algebra with unit element $e$ which is invariant under the involution $Q_{e}$. The 1-eigenspace

$$
Z^{+}:=\left\{x \in Z^{1}: Q_{e} x=x\right\}
$$

is a euclidean Jordan algebra of rank $r$ with unit element $e$. Using the "joint" Peirce decomposition with respect to a frame $[\mathrm{L}$, one defines numerical invariants $a, b$ satisfying

The genus is defined as

$$
\begin{gathered}
\ell_{\mathbf{C}}:=\frac{\operatorname{dim} Z^{1}}{r}=1+\frac{a}{2}(r-1), \\
\frac{\operatorname{dim} Z^{1 / 2}}{r}=b .
\end{gathered}
$$

$$
p:=2+a(r-1)+b=\ell_{\mathbf{C}}+\frac{d}{r} .
$$

We define the "normalized" inner product

$$
(z \mid w):=\frac{1}{p} \operatorname{tr} D(z, w) .
$$

Any maximal tripotent $e \in Z_{\mathbf{K}}$ is also maximal in $Z$. Its rank $r_{\mathbf{K}}$ relative to $Z_{\mathbf{K}}$ may be different from $r$ since minimal tripotents in $Z_{\mathbf{K}}$ may not be minimal in $Z$. The Peirce decomposition

$$
Z_{\mathbf{K}}=Z_{\mathbf{K}}^{1} \oplus Z_{\mathbf{K}}^{1 / 2}
$$

yields a semi-simple real Jordan algebra $Z_{\mathbf{K}}^{1}$ which has a splitting

$$
Z_{\mathbf{K}}^{1}=Z_{\mathbf{K}}^{+} \oplus Z_{\mathbf{K}}^{-} \text {, }
$$

where $Z_{\mathbf{K}}^{ \pm}$is the \pm 1-eigenspace of the involution $Q_{e}$ on $Z_{\mathbf{K}}^{1}$. One can show that $Z_{\mathbf{K}}^{+}$ is a euclidean Jordan algebra of rank $r_{\mathbf{K}}$. The joint Peirce decomposition $[\mathrm{L}]$ of $Z_{\mathbf{K}}$ defines numerical invariants $a_{\mathbf{K}}, b_{\mathbf{K}}, c_{\mathbf{K}}$ satisfying

$$
\begin{gathered}
\ell_{\mathbf{K}}:=\frac{\operatorname{dim} Z_{\mathbf{K}}^{+}}{r_{\mathbf{K}}}=1+\frac{a_{\mathbf{K}}}{2}\left(r_{\mathbf{K}}-1\right), \\
\frac{\operatorname{dim} Z_{\mathbf{K}}^{1 / 2}}{r_{\mathbf{K}}}=b_{\mathbf{K}}, \\
\frac{\operatorname{dim} Z_{\mathbf{K}}^{-}}{r_{\mathbf{K}}}=c_{\mathbf{K}}+\frac{a_{\mathbf{K}}}{2}\left(r_{\mathbf{K}}-1\right) .
\end{gathered}
$$

Here we have to exclude the so-called root type (D2) which will not be considered further. Put

$$
\kappa:=\operatorname{dim}_{\mathbf{R}} \mathbf{K}
$$

The "real" genus

$$
p_{\mathbf{K}}:=1+c_{\mathbf{K}}+a_{\mathbf{K}}\left(r_{\mathbf{K}}-1\right)+b_{\mathbf{K}} / 2=\ell_{\mathbf{K}}+\frac{\kappa d}{2 r_{\mathbf{K}}}-\frac{1-c_{\mathbf{K}}}{2}
$$

satisfies

$$
\frac{r_{\mathbf{K}}}{r} p_{\mathbf{K}}=\frac{\kappa}{2} p .
$$

Define an inner product on $Z_{\mathbf{K}}$ by

$$
(x \mid y)_{\mathbf{K}}:=\frac{r_{\mathbf{K}}}{r} \operatorname{Re}(x \mid y)
$$

for all $x, y \in Z_{\mathbf{K}} \subset Z$. 
The open unit ball $\check{Z}$ of $Z$ for the so-called spectral norm is an irreducible hermitian bounded symmetric domain, and all such domains arise this way $\underline{\mathrm{L}}$. The conformal compactification $\hat{Z}$ of $Z$, described in Jordan theoretic terms in $[\mathrm{L}$, is the compact dual space of $\check{Z}$. For a uniform treatment of the various types we let $\dot{Z}$ denote any of the hermitian symmetric spaces $Z$ (flat case), $\check{Z}$ (bounded case), or $\hat{Z}$ (compact case). In the real case the unit ball $\check{Z}_{\mathbf{R}}=Z_{\mathbf{R}} \cap \check{Z}$ is an irreducible real bounded symmetric domain and the compact dual space of $\check{Z}_{\mathbf{R}}$ is the conformal compactification $\hat{Z}_{\mathbf{R}} \subset \hat{Z}$. We denote by $\dot{Z}_{\mathbf{R}}$ any of the spaces $Z_{\mathbf{R}}$ (flat case), $\check{Z}_{\mathbf{R}}$ (bounded case), or $\hat{Z}_{\mathbf{R}}$ (compact case). In the complex case $\dot{Z}_{\mathbf{C}}$ is the real symmetric space underlying $\dot{Z}$. One can show $[\mathrm{L}$ that every irreducible real bounded symmetric domain has the form $\check{Z}_{\mathbf{K}}$ and the spaces $\hat{Z}_{\mathbf{K}}$ comprise all irreducible compact symmetric R-spaces $[\mathrm{L}$.

Using (2.1) we define the Bergman operator

$$
B(z, w):=I-D(z, w)+Q_{z} Q_{w} .
$$

A pair $(z, w)$ is called quasi-invertible if $B(z, w)$ is invertible. In this case

$$
z^{w}:=B(z, w)^{-1}\left(z-Q_{z} w\right)
$$

is called the quasi-inverse. Let $\tilde{K} \subset G L(Z)$ denote the complex Lie group of "Jordan pair" automorphisms of $Z$, generated by all operators $B(u, v)$ with $(u, v)$ quasi-invertible. The compact subgroup $K:=\left\{k \in \tilde{K}: k^{*}=k^{-1}\right\}$ of $\tilde{K}$ consists of all "Jordan triple automorphisms". The conformal group $\tilde{G}$ of $Z$ is generated by $\tilde{K}$ and two types of non-linear transformations: The translations $\mathfrak{t}_{z}(z \in Z)$ given by

$$
\mathfrak{t}_{z} w:=z+w
$$

for $w \in Z \subset \hat{Z}$, and their "adjoints" $\mathfrak{t}_{z}^{*}(z \in Z)$ given by

$$
\mathfrak{t}_{z}^{*} w:=w^{-z}
$$

for $w \in Z$ such that $(w,-z)$ is quasi-invertible (a dense open subset of $Z \subset \hat{Z}$ ). One can show that $\tilde{G}$ coincides with the identity component of the bi-holomorphic automorphism group of $\hat{Z}$. The transvections $\dot{\mathfrak{t}}_{z}$ are defined by

$$
\check{\mathfrak{t}}_{z}:=\mathfrak{t}_{z} B(z, z)^{1 / 2} \mathfrak{t}_{z}^{*}
$$

in the non-compact case and

$$
\hat{\mathfrak{t}}_{z}:=\mathfrak{t}_{z} B(z,-z)^{1 / 2} \mathfrak{t}_{-z}^{*}
$$

in the compact case. In the flat case, transvections and translations coincide. We often use the $\epsilon$-convention: $\epsilon=1$ (bounded case), $\epsilon=0$ (flat case) and $\epsilon=$ -1 (compact case) to express formulas in a uniform manner. For example, the transvections can be written as

$$
\dot{\mathfrak{t}}_{z}:=\mathfrak{t}_{z} B(z, \epsilon z)^{1 / 2} \mathfrak{t}_{\epsilon z}^{*} .
$$

Let $\dot{G}$ denote the subgroup of $\tilde{G}$ generated by $K$ and the transvections $\dot{\mathfrak{t}}_{z}$, for $z \in Z$. For the flat type

$$
G \approx K \times Z
$$

is a semi-direct product, whereas $\hat{G}$ is a maximal compact subgroup of $\tilde{G}$ and $\check{G}$ is the (connected) bi-holomorphic automorphism group of $\check{Z}$. In all three cases $\dot{G}$ acts transitively on $\dot{Z}$, with stabilizer subgroup $K$ at the origin. Therefore

$$
\dot{Z} \approx \dot{G} / K \text {. }
$$


One can show that $\tilde{G} \approx \dot{G}^{\mathbf{C}}$ and $\tilde{K} \approx K^{\mathbf{C}}$ as complex Lie groups. Let $\dot{G}_{\mathbf{R}} \subset \dot{G}$ be the subgroup consisting of all $g \in \dot{G}$ which satisfy

$$
g\left(z^{\sharp}\right)=(g(z))^{\sharp},
$$

and put $\dot{G}_{\mathbf{C}}:=\dot{G}$, considered as a group of real isometries of $\dot{Z}$. Then $\dot{G}_{\mathbf{K}}$ is generated by $K_{\mathbf{K}}:=K \cap \dot{G}_{\mathbf{K}}$ and the transvections $\dot{\mathfrak{t}}_{x}$, for $x \in Z_{\mathbf{K}}$. For the flat type

$$
G_{\mathbf{K}} \approx K_{\mathbf{K}} \times Z_{\mathbf{K}}
$$

is a semi-direct product, whereas $\hat{G}_{\mathbf{K}}$ is a maximal compact subgroup of the conformal group $\tilde{G}_{\mathbf{K}}$ and $\check{G}_{\mathbf{K}}$ is the (reductive) Lie group associated to the real bounded symmetric domain $\check{Z}_{\mathbf{K}}$. Since $\dot{G}_{\mathbf{K}}$ acts transitively on $\dot{Z}_{\mathbf{K}}$, with stabilizer subgroup $K_{\mathbf{K}}$ at the origin, it follows that

$$
\dot{Z}_{\mathbf{K}} \approx \dot{G}_{\mathbf{K}} / K_{\mathbf{K}}
$$

\section{HolOMORPHIC FUNCTION SPACES AND UNITARY REPRESENTATIONS}

Let $Z$ be an irreducible hermitian Jordan triple of rank $r$, multiplicity $a$, dimension $d$ and genus $p$. One can show that

$$
\operatorname{det} B(z, w)=\Delta(z, w)^{p}
$$

for some (inhomogeneous) sesqui-polynomial $\Delta$ called the Jordan triple determinant. For $z, w \in \dot{Z}$ define sesqui-holomorphic functions

$$
\dot{K}(z, w):=\exp \left(z \mid\left(1-\epsilon^{2}\right) w\right) \Delta(z, \epsilon w)^{-\epsilon} .
$$

A more intuitive formula will be given in (5.3). Specifically,

$$
\begin{gathered}
K(z, w)=\exp (z \mid w), \\
\check{K}(z, w)=\Delta(z, w)^{-1}, \\
\hat{K}(z, w)=\Delta(z,-w) .
\end{gathered}
$$

Here and in the sequel it is tacitly understood that in the compact case one restricts to the local chart $Z \subset \hat{Z}$. In particular,

$$
\dot{K}(z, w)^{-\epsilon}=\Delta(z, \epsilon w) .
$$

Let $\Omega$ denote the symmetric cone $\left[\mathrm{FK}\right.$ of $Z^{+}$. The Gindikin-Gamma function is defined by

$$
\Gamma_{\Omega}(\boldsymbol{m})=(2 \pi)^{\operatorname{ar}(r-1) / 4} \prod_{j=1}^{r} \Gamma\left(m_{j}-\frac{a}{2}(j-1)\right)
$$

for all complex $r$-tuples $\boldsymbol{m}=\left(m_{1}, \ldots, m_{r}\right)$. Consider the polynomial algebra $\mathcal{P}(Z)$. Every $p \in \mathcal{P}(Z)$ gives rise to a constant coefficient holomorphic differential operator $p(\partial)$ uniquely determined by the property

$$
p(\partial) e^{(z \mid a)}=\overline{p(a)} e^{(z \mid a)}
$$

for all $a \in Z$. Note that $p(\partial)$ is anti-linear in $p$. Then

$$
(f \mid p)_{F}:=(p(\partial) f)(0)
$$

is the Fischer-Fock inner product on $\mathcal{P}(Z)$. By [FK] there is a Peter-Weyl decomposition

$$
\mathcal{P}(Z)=\sum_{\boldsymbol{m}} \mathcal{P}_{\boldsymbol{m}}(Z)
$$


into irreducible $K$-modules $\mathcal{P}_{\boldsymbol{m}}(Z)$ labelled by all integer partitions

$$
\boldsymbol{m}=\left(m_{1}, \ldots, m_{r}\right)
$$

with $m_{1} \geq m_{2} \geq \cdots \geq m_{r} \geq 0$. Let

$$
\exp (z \mid w)=\sum_{\boldsymbol{m}} E_{\boldsymbol{m}}(z, w)
$$

be the corresponding expansion of the Fischer-Fock reproducing kernel into the reproducing kernel functions $E_{\boldsymbol{m}}(z, w)$ of $\mathcal{P}_{\boldsymbol{m}}(Z)$. More explicitly, we put $d_{\boldsymbol{m}}:=$ $\operatorname{dim} \mathcal{P}_{\boldsymbol{m}}(Z)$ and choose an orthonormal basis $\left(u_{\boldsymbol{m}}^{i}\right)_{1 \leq i \leq d_{\boldsymbol{m}}}$ of $\mathcal{P}_{\boldsymbol{m}}(Z)$. Then

$$
E_{\boldsymbol{m}}(z, w)=\sum_{i=1}^{d_{\boldsymbol{m}}} u_{\boldsymbol{m}}^{i}(z) \overline{u_{\boldsymbol{m}}^{i}(w)}
$$

for all $z, w \in Z$. The well-known Faraut-Korányi formula [FK] asserts that

$$
\Delta(z, w)^{-n}=\sum_{\boldsymbol{m}}(n)_{\boldsymbol{m}} E_{\boldsymbol{m}}(z, w)
$$

for all $n \in \mathbf{C}$. Here

$$
(n)_{\boldsymbol{m}}=\frac{\Gamma_{\Omega}(n+\boldsymbol{m})}{\Gamma_{\Omega}(n)}
$$

is the multi-variable Pochhammer symbol. Let $d \lambda(z)$ denote the Lebesgue measure for the inner product (2.2). Then

$$
d \dot{\lambda}(z):=d \lambda(z) \dot{K}(z, z)^{\epsilon p}=d \lambda(z) \Delta(z, \epsilon z)^{-p}
$$

is a $\dot{G}$-invariant measure on $\dot{Z}$. Choose a scalar parameter $n$ satisfying $n>0$ in the flat case, $n>p-1$ in the bounded case, and $n \in \mathbf{N}$ in the compact case. Define normalization constants $\dot{C}_{n}$ by

$$
\begin{gathered}
\pi^{d} C_{n}:=n^{d}, \\
\pi^{d} \check{C}_{n}:=\frac{\Gamma_{\Omega}(n)}{\Gamma_{\Omega}\left(n-\frac{d}{r}\right)}, \\
\pi^{d} \hat{C}_{n}:=\frac{\Gamma_{\Omega}(n+p)}{\Gamma_{\Omega}\left(n+p-\frac{d}{r}\right)}=\frac{\Gamma_{\Omega}\left(n+\ell_{\mathbf{C}}+\frac{d}{r}\right)}{\Gamma_{\Omega}\left(n+\ell_{\mathbf{C}}\right)} .
\end{gathered}
$$

Let $L_{n}^{2}(\dot{Z})$ denote the $L^{2}$-space for the $n$-th probability measure on $\dot{Z}$ given by

$$
\dot{C}_{n} \dot{K}(z, z)^{-n} d \dot{\lambda}(z)=\dot{C}_{n} \dot{K}(z, z)^{\epsilon p-n} d \lambda(z) .
$$

This measure is $K$-invariant but not $\dot{G}$-invariant unless $n=0$ for the compact type. The quantization Hilbert space $H_{n}^{2}(\dot{Z}) \subset L_{n}^{2}(\dot{Z})$ is defined as the closed subspace spanned by all "kernel" functions

$$
z \mapsto \dot{K}_{w}^{n}(z)=\dot{K}_{n}(z, w):=\dot{K}(z, w)^{n},
$$

where $w \in \dot{Z}$. In the flat case, we obtain the weighted Fock space of entire functions. In the bounded case, these are the weighted Bergman spaces of holomorphic functions. In the compact case, where $n \in \mathbf{N}$, there are no non-constant holomorphic functions and $H_{n}^{2}(\hat{Z})$ is best regarded as a (finite dimensional) space of holomorphic sections of the $n$-th power of a suitable line bundle over $\hat{Z}$. Restricting to the local chart $Z \subset \hat{Z}$, we may identify $H_{n}^{2}(\hat{Z})$ with the linear span of all polynomials $z \mapsto \Delta_{w}^{n}(z):=\Delta(z, w)^{n}$, where $w \in Z$ is arbitrary. In the rank 1 case we obtain all 
polynomials of total degree $\leq n$. For higher rank the situation is more complicated. Since $(-n)_{m}=0$ if and only if $m_{1}>n$, applying (3.5) to $-n$ yields

$$
H_{n}^{2}(\hat{Z}) \approx \sum_{\boldsymbol{m} \subset r \times n} \mathcal{P}_{\boldsymbol{m}}(Z),
$$

with $\boldsymbol{m}$ ranging over all partitions (3.3) satisfying $m_{1} \leq n$, i.e., with the Young diagram contained in the rectangle $r \times n$.

One can show that the functions (3.7) have the reproducing kernel property: any $\psi \in H_{n}^{2}(\dot{Z})$ can be written in the form

$$
\psi=\dot{C}_{n} \int_{\dot{Z}} d \dot{\lambda}(w) \dot{K}(w, w)^{-n} \psi(w) \dot{K}_{w}^{n} .
$$

Thus the orthogonal projection $\dot{P}_{n}$ onto $H_{n}^{2}(\dot{Z})$ is given by

$$
\begin{aligned}
\left(\dot{P}_{n} \psi\right)(z) & =\dot{C}_{n} \int_{\dot{Z}} d \dot{\lambda}(w) \dot{K}(w, w)^{-n} \dot{K}_{n}(z, w) \psi(w) \\
& =\dot{C}_{n} \int_{\dot{Z}} d \lambda(w) \Delta(w, \epsilon w)^{-p} \frac{\dot{K}(z, w)^{n}}{\dot{K}(w, w)^{n}} \psi(w) \\
& =\dot{C}_{n} \int_{\dot{Z}} d \lambda(w) \frac{\dot{K}(z, w)^{n}}{\dot{K}(w, w)^{n-\epsilon p}} \psi(w)
\end{aligned}
$$

or, more explicitly,

$$
\begin{gathered}
\left(P_{n} \psi\right)(z)=\left(\frac{n}{\pi}\right)^{d} \int_{Z} d \lambda(w)(\exp (z-w \mid w))^{n} \psi(w), \\
\left(\check{P}_{n} \psi\right)(z)=\frac{\Gamma_{\Omega}(n)}{\pi^{d} \Gamma_{\Omega}\left(n-\frac{d}{r}\right)} \int_{\check{Z}} d \lambda(w) \frac{\Delta(z, w)^{-n}}{\Delta(w, w)^{p-n}} \psi(w), \\
\left(\hat{P}_{n} \psi\right)(z)=\frac{\Gamma_{\Omega}(n+p)}{\pi^{d} \Gamma_{\Omega}\left(n+p-\frac{d}{r}\right)} \int_{Z} d \lambda(w) \frac{\Delta(z,-w)^{n}}{\Delta(w,-w)^{p+n}} \psi(w) .
\end{gathered}
$$

The symmetric space $\dot{Z}$ carries a tangent metric given by

$$
(u \mid v)_{z}=\left(u \mid B(z, \epsilon z)^{-1} v\right),
$$

where $z \in \dot{Z}$ and $u, v \in T_{z}(\dot{Z}) \approx Z$ belong to the tangent space. Every $g \in \dot{G}$ satisfies

$$
g^{\prime}(z) B(z, \epsilon w) g^{\prime}(w)^{*}=B(g z, \epsilon g w)
$$

for all $z, w \in \dot{Z}$, as follows from $g^{\prime}(z)^{*} B(g z, \epsilon g z)^{-1} g^{\prime}(z)=B(z, \epsilon z)^{-1}$ by polarization. With (3.1) it follows that

$$
\operatorname{det} g^{\prime}(z) \Delta(z, \epsilon w)^{p} \overline{\operatorname{det} g^{\prime}(w)}=\Delta(g z, \epsilon g w)^{p} .
$$

In the compact case, the identity (3.12) holds for a dense open subset in the local chart $Z \subset \hat{Z}$. This point will usually be tacitly understood in the following.

Proposition 3.1. There exists an irreducible unitary (projective) representation $g \mapsto \dot{U}_{n}(g)$ of $\dot{G}$ on $H_{n}^{2}(\dot{Z})$ defined by

$$
\begin{gathered}
\left(U_{n}\left(g^{-1}\right) \psi\right)(z)=\left(\exp \left(g(z)-\frac{g(0)}{2} \mid g(0)\right)\right)^{-n} \psi(g(z)), \\
\left(\check{U}_{n}\left(g^{-1}\right) \psi\right)(z)=\operatorname{det} g^{\prime}(z)^{n / p} \psi(g(z)), \\
\left(\hat{U}_{n}\left(g^{-1}\right) \psi\right)(z)=\operatorname{det} g^{\prime}(z)^{-n / p} \psi(g(z))
\end{gathered}
$$

for (almost) all $z \in \dot{Z}$ and $\psi \in H_{n}^{2}(\dot{Z})$. 
Proof. In the bounded setting, this is the scalar holomorphic discrete series of the semi-simple Lie group $\check{G}$. For the flat type we obtain the Schrödinger representation in the complex wave setting. For the compact type we first show that for each $g \in \tilde{G}$, the function $z \mapsto \operatorname{det} g^{\prime}(z)$ has a holomorphic $p$-th root on the open dense subset

$$
\Omega_{g}:=\{z \in Z: g(z) \in Z\} \subset Z .
$$

Every $g \in \tilde{G}$ satisfying $g(0) \in Z$ has a unique decomposition

$$
g=\mathfrak{t}_{x} h \mathfrak{t}_{y}^{*},
$$

where $x, y \in Z$ and $h \in \tilde{K}$. By [L, Proposition 8.10], we may write

$$
g=\mathfrak{t}_{x} h \mathfrak{t}_{y}^{*} \mathfrak{t}_{w}
$$

for $x, y, w \in Z$ and $h \in \tilde{K}$. Now let $z \in \Omega_{g}$. Then $g(z) \in Z$ and hence

$$
(z+w)^{-y}=\mathfrak{t}_{y}^{*} \mathfrak{t}_{w} z=h^{-1}(g(z)-x) \in Z .
$$

It follows that $(z+w,-y)$ is quasi-invertible. Therefore

$$
g^{\prime}(z)=h\left(\mathfrak{t}_{y}^{*}\right)^{\prime}(z+w)=h B(z+w,-y)^{-1} .
$$

Choosing generators of $\tilde{K}$ we obtain an expression

$$
g^{\prime}(z)=B\left(u_{1}, v_{1}\right) \cdots B\left(u_{l}, v_{l}\right) B(z+w,-y)^{-1}
$$

for quasi-invertible pairs $\left(u_{1}, v_{1}\right), \ldots,\left(u_{l}, v_{l}\right)$. Therefore

$$
\operatorname{det} g^{\prime}(z)=\Delta\left(u_{1}, v_{1}\right)^{p} \cdots \Delta\left(u_{l}, v_{l}\right)^{p} \Delta(z+w,-y)^{-p} .
$$

Define

$$
\operatorname{det} g^{\prime}(z)^{1 / p}:=\Delta\left(u_{1}, v_{1}\right) \cdots \Delta\left(u_{l}, v_{l}\right) \Delta(z+w,-y)^{-1} .
$$

By (3.13) there exists a $p$-th root of unity $\vartheta$ depending on $g$ such that

$$
\operatorname{det} g^{\prime}(z)^{-n / p} \Delta(g z,-g w)^{n}=\vartheta{\overline{\operatorname{det} g^{\prime}(w)}}^{n / p} \Delta(z,-w)^{n}
$$

for all $z, w \in \Omega_{g}$. Therefore

$$
\hat{U}_{n}\left(g^{-1}\right) \Delta_{-g w}^{n}=\vartheta{\overline{\operatorname{det} g^{\prime}(w)}}^{n / p} \Delta_{-w}^{n}
$$

for all $w \in \Omega_{g}$. Since $H_{n}^{2}(\hat{Z})$ is spanned by $\left\{\Delta_{-g w}^{n}: w \in \Omega_{g}\right\}$, the assertion follows again by (3.13).

For the real form $Z_{\mathbf{K}}$ let $d \lambda_{\mathbf{K}}(x)$ be the Lebesgue measure associated with (2.4). Define $\dot{G}_{\mathbf{K}}$ invariant measures on $\dot{Z}_{\mathbf{K}}$ by

$$
d \dot{\lambda}_{\mathbf{K}}(x)=\dot{K}(x, x)^{\epsilon p \kappa / 2} d \lambda_{\mathbf{K}}(x)=\Delta(x, \epsilon x)^{-p \kappa / 2} d \lambda_{\mathbf{K}}(x) .
$$

Given the scalar parameter $n$ specified above we define the associated "real" parameter $\nu$ by

$$
\frac{r_{\mathbf{K}}}{r} \nu=\frac{\kappa}{2} n
$$

Let $\Omega_{\mathbf{K}}$ denote the symmetric cone of rank $r_{\mathbf{K}}$ associated with $Z_{\mathbf{K}}^{+}$. 
Proposition 3.2. For any $x$ and integer partition $\boldsymbol{\mu}=\left(\mu_{1}, \ldots, \mu_{r_{\mathbf{K}}}\right)$ we have the reciprocity

$$
(-1)^{\boldsymbol{\mu}} \frac{\Gamma_{\Omega_{\mathbf{K}}}(x)}{\Gamma_{\Omega_{\mathbf{K}}}(x-\boldsymbol{\mu})}=\left(\ell_{\mathbf{K}}-x\right)_{\boldsymbol{\mu}^{*}}
$$

where $\boldsymbol{\mu}^{*}:=\left(\mu_{r_{\mathbf{K}}}, \ldots, \mu_{1}\right)$.

Proof. Using $(-1)^{m} \frac{\Gamma(x)}{\Gamma(x-m)}=(1-x)_{m}$ and writing

it follows that

$$
1+\frac{a_{\mathbf{K}}}{2}(j-1)=\ell_{\mathbf{K}}-\frac{a_{\mathbf{K}}}{2}\left(r_{\mathbf{K}}-j\right),
$$

$$
\begin{gathered}
(-1)^{|\boldsymbol{\mu}|} \frac{\Gamma_{\Omega_{\mathbf{K}}}(x)}{\Gamma_{\Omega_{\mathbf{K}}}(x-\boldsymbol{\mu})}=\prod_{j=1}^{r_{\mathbf{K}}}(-1)^{\mu_{j}} \frac{\Gamma\left(x-\frac{a_{\mathbf{K}}}{2}(j-1)\right.}{\Gamma\left(x-\frac{a_{\mathbf{K}}}{2}(j-1)-\mu_{j}\right)}=\prod_{j=1}^{r_{\mathbf{K}}}\left(\frac{a_{\mathbf{K}}}{2}(j-1)-x+1\right)_{\mu_{j}} \\
=\prod_{j=1}^{r_{\mathbf{K}}}\left(\ell_{\mathbf{K}}-x-\frac{a_{\mathbf{K}}}{2}\left(r_{\mathbf{K}}-j\right)\right)_{\mu_{j}}=\prod_{j=1}^{r_{\mathbf{K}}}\left(\ell_{\mathbf{K}}-x-\frac{a_{\mathbf{K}}}{2}(j-1)\right)_{\mu_{j}^{*}} \\
=\frac{\Gamma_{\Omega_{\mathbf{K}}}\left(\ell_{\mathbf{K}}-x+\boldsymbol{\mu}^{*}\right)}{\Gamma_{\Omega_{\mathbf{K}}}\left(\ell_{\mathbf{K}}-x\right)}=\left(\ell_{\mathbf{K}}-x\right)_{\boldsymbol{\mu}^{*}} .
\end{gathered}
$$

Choose the normalization constants $\dot{C}_{\mathbf{K}}^{\nu}$ by putting

$$
\begin{gathered}
\pi^{\kappa d / 2} C_{\mathbf{K}}^{\nu}:=\nu^{\kappa d / 2} \\
\pi^{\kappa d / 2} \check{C}_{\mathbf{K}}^{\nu}:=\frac{\Gamma_{\Omega_{\mathbf{K}}}\left(\nu+\frac{1-c_{\mathbf{K}}}{2}\right)}{\Gamma_{\Omega_{\mathbf{K}}}\left(\nu+\frac{1-c_{\mathbf{K}}}{2}-\frac{\kappa d}{2 r_{\mathbf{K}}}\right)}=\frac{\Gamma_{\Omega_{\mathbf{K}}}\left(\nu+\frac{1-c_{\mathbf{K}}}{2}\right)}{\Gamma_{\Omega_{\mathbf{K}}}\left(\nu+\ell_{\mathbf{K}}-p_{\mathbf{K}}\right)}, \\
\pi^{\kappa d / 2} \hat{C}_{\mathbf{K}}^{\nu}:=\frac{\Gamma_{\Omega_{\mathbf{K}}}\left(\nu+p_{\mathbf{K}}\right)}{\Gamma_{\Omega_{\mathbf{K}}}\left(\nu+p_{\mathbf{K}}-\frac{\kappa d}{2 r_{\mathbf{K}}}\right)}=\frac{\Gamma_{\Omega_{\mathbf{K}}}\left(\nu+p_{\mathbf{K}}\right)}{\Gamma_{\Omega_{\mathbf{K}}}\left(\nu+\ell_{\mathbf{K}}-\frac{1-c_{\mathbf{K}}}{2}\right)} .
\end{gathered}
$$

For the compact type we may take $n=0$, and

$$
\hat{C}_{\mathbf{K}}^{0} d \hat{\lambda}_{\mathbf{K}}(x)=\hat{C}_{\mathbf{K}}^{0} \hat{K}(x, x)^{\epsilon p \kappa / 2} d \lambda_{\mathbf{K}}(x)=\frac{\Gamma_{\Omega_{\mathbf{K}}}\left(p_{\mathbf{K}}\right)}{\pi^{\kappa d / 2} \Gamma_{\Omega_{\mathbf{K}}}\left(p_{\mathbf{K}}-\frac{\kappa d}{2 r_{\mathbf{K}}}\right)} \Delta(x,-x)^{-p \kappa / 2} d \lambda_{\mathbf{K}}(x)
$$

is the $\hat{G}_{\mathbf{K}}$ invariant normalized Haar measure on $\hat{Z}_{\mathbf{K}}$.

The complexification of $Z_{\mathbf{K}}$ will be denoted by $Z^{\mathbf{K}} \approx Z^{\kappa}$. As a rule we will use the superscript $\mathbf{K}=\mathbf{R}, \mathbf{C}$ for objects associated with the complexification. Thus

$$
\begin{array}{ll}
Z^{\mathbf{R}}=Z, & Z^{\mathbf{C}}=Z \times \bar{Z}=\left\{\left(z_{1}, \bar{z}_{2}\right): z_{1}, z_{2} \in Z\right\}, \\
\dot{Z}^{\mathbf{R}}=\dot{Z}, & \dot{Z}^{\mathbf{C}}=\dot{Z} \times \bar{Z}=\left\{\left(z_{1}, \bar{z}_{2}\right): z_{1}, z_{2} \in \dot{Z}\right\},
\end{array}
$$

where the conjugation $\left(z_{1}, \bar{z}_{2}\right)^{\sharp}=\left(z_{2}, \bar{z}_{1}\right)$ of $\dot{Z}^{\mathbf{C}}$ has the fixed point set $\dot{Z}_{\mathbf{C}}$ under the diagonal embedding $\dot{Z}_{\mathbf{C}} \subset \dot{Z}^{\mathbf{C}}: z \mapsto(z, \bar{z})$. Moreover,

$$
\begin{gathered}
(z \mid w)^{\mathbf{R}}:=(z \mid w), \quad\left(z_{1}, \bar{z}_{2} \mid w_{1}, \bar{w}_{2}\right)^{\mathbf{C}}:=\left(z_{1} \mid w_{1}\right)+\left(w_{2} \mid z_{2}\right), \\
\Delta^{\mathbf{R}}(z, w)=\Delta(z, w), \quad \Delta^{\mathbf{C}}\left(z_{1}, \bar{z}_{2}, w_{1}, \bar{w}_{2}\right)=\Delta\left(z_{1}, w_{1}\right) \Delta\left(w_{2}, z_{2}\right), \\
\\
d \lambda^{\mathbf{R}}(z)=d \lambda(z), \quad d \lambda^{\mathbf{C}}(z, \bar{w})=d \lambda(z) d \lambda(w), \\
\dot{K}^{\mathbf{R}}(z, w)=\dot{K}(z, w), \quad \dot{K}^{\mathbf{C}}\left(z_{1}, \bar{z}_{2}, w_{1}, \bar{w}_{2}\right)=\dot{K}\left(z_{1}, w_{1}\right) \dot{K}\left(w_{2}, z_{2}\right), \\
d \dot{\lambda}^{\mathbf{R}}(z)=d \dot{\lambda}(z), \quad d \dot{\lambda}^{\mathbf{C}}(z, \bar{w})=d \dot{\lambda}(z) d \dot{\lambda}(w),
\end{gathered}
$$




$$
\begin{aligned}
& H_{n}^{2}\left(\dot{Z}^{\mathbf{R}}\right)=H_{n}^{2}(\dot{Z}), \quad H_{n}^{2}\left(\dot{Z}^{\mathbf{C}}\right)=H_{n}^{2}(\dot{Z} \times \overline{\dot{Z}})=H_{n}^{2}(\dot{Z}) \otimes \overline{H_{n}^{2}(\dot{Z})}, \\
& \dot{K}_{n}^{\mathbf{R}}(z, w)=\dot{K}_{n}(z, w), \quad \dot{K}_{n}^{\mathbf{C}}\left(z_{1}, \bar{z}_{2}, w_{1}, \bar{w}_{2}\right)=\dot{K}_{n}\left(z_{1}, w_{1}\right) \dot{K}_{n}\left(w_{2}, z_{2}\right) .
\end{aligned}
$$

Note that $Z^{\mathbf{C}}$ has only one real form $Z_{\mathbf{C}}$, but there may be several real forms $Z_{\mathbf{R}}$ with the same complexification $Z^{\mathbf{R}}=Z$. For $x \in Z_{\mathbf{K}} \subset Z$ we have

$$
\begin{gathered}
\Delta^{\mathbf{K}}(x, \epsilon x)=\Delta(x, \epsilon x)^{\kappa}, \\
\Delta^{\mathbf{K}}(x, \epsilon x)^{p / 2}=\Delta(x, \epsilon x)^{p \kappa / 2}=\Delta(x, \epsilon x)^{p_{\mathbf{K}} r_{\mathbf{K}} / r}, \\
\dot{K}^{\mathbf{K}}(x, x)=\dot{K}(x, x)^{\kappa}, \\
\dot{K}_{n}^{\mathbf{K}}(x, x)^{1 / 2}=\dot{K}(x, x)^{n \kappa / 2}=\dot{K}(x, x)^{\nu r_{\mathbf{K}} / r} .
\end{gathered}
$$

\section{Toeplitz operators AND Berezin transform}

This paper is devoted to a systematic study, using asymptotic expansions, of the well-known Toeplitz-Berezin quantization for symmetric spaces of any type, including a generalization to real symmetric spaces. In this section we concentrate on the formal properties and give rigorous definitions of Toeplitz-Berezin quantization.

Definition 4.1. The Berezin symbol map is the densely defined operator

$$
\dot{\sigma}_{\mathbf{K}}^{\nu}: H_{n}^{2}\left(\dot{Z}^{\mathbf{K}}\right) \longrightarrow L^{2}\left(\dot{Z}_{\mathbf{K}}, \dot{C}_{\mathbf{K}}^{\nu} \dot{\lambda}_{\mathbf{K}}\right)
$$

given by

$$
\left(\dot{\sigma}_{\mathbf{K}}^{\nu} h\right)(x):=\frac{h(x)}{\dot{K}_{n}^{\mathbf{K}}(x, x)^{1 / 2}}=\frac{h(x)}{\dot{K}(x, x)^{n \kappa / 2}}
$$

for $x \in \dot{Z}_{\mathbf{K}}$ and $h \in H_{n}^{2}\left(\dot{Z}^{\mathbf{K}}\right)$.

Proposition 4.2. The formally adjoint Toeplitz map

$$
\dot{\tau}_{\mathbf{K}}^{\nu}=\left(\dot{\sigma}_{\mathbf{K}}^{\nu}\right)^{*}: L^{2}\left(\dot{Z}_{\mathbf{K}}, \dot{C}_{\mathbf{K}}^{\nu} \dot{\lambda}_{\mathbf{K}}\right) \longrightarrow H_{n}^{2}\left(\dot{Z}^{\mathbf{K}}\right)
$$

has the form

$$
\begin{aligned}
\left(\dot{\tau}_{\mathbf{K}}^{\nu} f\right)(z) & =\dot{C}_{\mathbf{K}}^{\nu} \int_{\dot{Z}_{\mathbf{K}}} d \dot{\lambda}_{\mathbf{K}}(y) \frac{\dot{K}_{n}^{\mathbf{K}}(z, y)}{\dot{K}_{n}^{\mathbf{K}}(y, y)^{1 / 2}} f(y) \\
& =\dot{C}_{\mathbf{K}}^{\nu} \int_{\dot{Z}_{\mathbf{K}}} d \lambda(y) \dot{K}^{\mathbf{K}}(z, y)^{n} \dot{K}(y, y)^{(\epsilon p-n) \kappa / 2} f(y) \\
& =\dot{C}_{\mathbf{K}}^{\nu} \int_{\dot{Z}_{\mathbf{K}}} d \lambda(y) \Delta(y, \epsilon y)^{-p \kappa / 2} \frac{\dot{K}^{\mathbf{K}}(z, y)^{n}}{\dot{K}(y, y)^{n \kappa / 2}} f(y) .
\end{aligned}
$$

Proof. The $n$-th probability measure on $\dot{Z}^{\mathbf{K}}$ is given by $\dot{C}_{n}^{\kappa} d \dot{\lambda}^{\mathbf{K}}(z) \dot{K}^{\mathbf{K}}(z, z)^{-n}$. Applying (3.8), it follows that for $h \in H_{n}^{2}\left(\dot{Z}^{\mathbf{K}}\right)$ we have the reproducing property

$$
h(y)=\dot{C}_{n}^{\kappa} \int_{\dot{Z}^{\mathbf{K}}} d \dot{\lambda}^{\mathbf{K}}(z) \dot{K}^{\mathbf{K}}(z, z)^{-n} \dot{K}_{n}^{\mathbf{K}}(y, z) h(z)
$$

which implies

$$
\begin{gathered}
\dot{C}_{n}^{\kappa} \int_{\dot{Z}^{\mathbf{K}}} d \dot{\lambda}^{\mathbf{K}}(z) \dot{K}^{\mathbf{K}}(z, z)^{-n} \overline{h(z)}\left(\dot{\tau}_{\mathbf{K}}^{\nu} f\right)(z) \\
=\dot{C}_{\mathbf{K}}^{\nu} \int_{\dot{Z}_{\mathbf{K}}} d \dot{\lambda}_{\mathbf{K}}(y) \overline{\left(\dot{\sigma}_{\mathbf{K}}^{\nu} h\right)(y)} f(y)=\dot{C}_{\mathbf{K}}^{\nu} \int_{\dot{Z}_{\mathbf{K}}} d \dot{\lambda}_{\mathbf{K}}(y) \frac{\overline{h(y)}}{\dot{K}_{n}^{\mathbf{K}}(y, y)^{1 / 2}} f(y)
\end{gathered}
$$




$$
\begin{aligned}
= & \dot{C}_{\mathbf{K}}^{\nu} \dot{C}_{n}^{\kappa} \int_{\dot{Z}_{\mathbf{K}}} d \dot{\lambda}_{\mathbf{K}}(y) \overline{\int_{\dot{Z}^{\mathbf{K}}} d \dot{\lambda}^{\mathbf{K}}(z) \dot{K}^{\mathbf{K}}(z, z)^{-n} \dot{K}_{n}^{\mathbf{K}}(y, z) h(z)} \frac{f(y)}{\dot{K}_{n}^{\mathbf{K}}(y, y)^{1 / 2}} \\
& =\dot{C}_{n}^{\kappa} \dot{C}_{\mathbf{K}}^{\nu} \int_{\dot{Z}^{\mathbf{K}}} d \dot{\lambda}^{\mathbf{K}}(z) \dot{K}^{\mathbf{K}}(z, z)^{-n} \overline{h(z)} \int_{\dot{Z}_{\mathbf{K}}} d \dot{\lambda}_{\mathbf{K}}(y) \frac{\dot{K}_{n}^{\mathbf{K}}(z, y)}{\dot{K}_{n}^{\mathbf{K}}(y, y)^{1 / 2}} f(y) .
\end{aligned}
$$

Definition 4.3. The Berezin transform $\dot{\beta}_{\mathbf{K}}^{\nu}:=\dot{\sigma}_{\mathbf{K}}^{\nu} \dot{\tau}_{\mathbf{K}}^{\nu}$ is the densely defined operator on $L^{2}\left(\dot{Z}_{\mathbf{K}}, \dot{C}_{\mathbf{K}}^{\nu} \dot{\lambda}_{\mathbf{K}}\right)$ given by

$$
\begin{aligned}
\left(\dot{\beta}_{\mathbf{K}}^{\nu} f\right)(x) & =\left(\dot{\sigma}_{\mathbf{K}}^{\nu} \dot{\tau}_{\mathbf{K}}^{\nu} f\right)(x)=\dot{C}_{\mathbf{K}}^{\nu} \int_{\dot{Z}_{\mathbf{K}}} d \dot{\lambda}_{\mathbf{K}}(y) \frac{\dot{K}_{n}^{\mathbf{K}}(x, y)}{\dot{K}_{n}^{\mathbf{K}}(x, x)^{1 / 2} \dot{K}_{n}^{\mathbf{K}}(y, y)^{1 / 2}} f(y) \\
& =\dot{C}_{\mathbf{K}}^{\nu} \int_{\dot{Z}_{\mathbf{K}}} d \lambda_{\mathbf{K}}(y) \frac{\dot{K}^{\mathbf{K}}(x, y)^{n}}{\dot{K}(x, x)^{n \kappa / 2} \dot{K}(y, y)^{(n-\epsilon p) \kappa / 2}} f(y) \\
= & \dot{C}_{\mathbf{K}}^{\nu} \int_{\dot{Z}_{\mathbf{K}}} d \lambda_{\mathbf{K}}(y) \Delta(y, \epsilon y)^{-p \kappa / 2} \frac{\dot{K}^{\mathbf{K}}(x, y)^{n}}{\dot{K}(x, x)^{n \kappa / 2} \dot{K}(y, y)^{n \kappa / 2}} f(y) .
\end{aligned}
$$

Specifically,

$$
\begin{gathered}
\left(\beta_{\mathbf{K}}^{\nu} f\right)(x)=C_{\mathbf{K}}^{\nu} \int_{Z_{\mathbf{K}}} d \lambda_{\mathbf{K}}(y) \frac{\exp \left(n(x \mid y)^{\mathbf{K}}\right)}{\exp \left(n \frac{\kappa}{2}(x \mid x)\right) \exp \left(n \frac{\kappa}{2}(y \mid y)\right)} f(y), \\
\left(\check{\beta}_{\mathbf{K}}^{\nu} f\right)(x)=\check{C}_{\mathbf{K}}^{\nu} \int_{\check{Z}_{\mathbf{K}}} d \lambda_{\mathbf{K}}(y) \frac{\Delta(x, x)^{n \kappa / 2} \Delta(y, y)^{\kappa(n-p) / 2}}{\Delta^{\mathbf{K}}(x, y)^{n}} f(y), \\
\left(\hat{\beta}_{\mathbf{K}}^{\nu} f\right)(x)=\hat{C}_{\mathbf{K}}^{\nu} \int_{Z_{\mathbf{K}}} d \lambda_{\mathbf{K}}(y) \frac{\Delta^{\mathbf{K}}(x,-y)^{n}}{\Delta(x,-x)^{n \kappa / 2} \Delta(y,-y)^{(n+p) \kappa / 2}} f(y) .
\end{gathered}
$$

Putting

$$
\dot{G}^{\mathbf{R}}=\dot{G}, \quad \dot{G}^{\mathbf{C}}=\dot{G} \times \overline{\dot{G}}=\left\{\left(g_{1}, \bar{g}_{2}\right): g_{1}, g_{2} \in \dot{G}\right\}
$$

we obtain a (real) Lie group $\dot{G}^{\mathbf{K}}$ acting on $\dot{Z}^{\mathbf{K}}$, such that $\dot{G}_{\mathbf{K}} \subset \dot{G}^{\mathbf{K}}$ is the fixed point group for the natural conjugation. Here $\dot{G}_{\mathbf{C}} \approx \dot{G} \subset \dot{G}^{\mathbf{C}}$ via the diagonal embedding $\dot{G} \ni g \mapsto(g, \bar{g}) \in \dot{G}^{\mathbf{C}}$. Define an irreducible unitary (projective) representation $\dot{U}_{n}^{\mathbf{K}}$ of $\dot{G}^{\mathbf{K}}$ on $H_{n}^{2}\left(\dot{Z}^{\mathbf{K}}\right)$ by

$$
\dot{U}_{n}^{\mathbf{R}}(g):=\dot{U}_{n}(g), \quad \dot{U}_{n}^{\mathbf{C}}\left(g_{1}, \bar{g}_{2}\right):=\dot{U}_{n}\left(g_{1}\right) \otimes \overline{\dot{U}_{n}\left(g_{2}\right)} .
$$

In general we denote by

$$
\left(T_{1} \otimes \bar{T}_{2}\right)(\phi \otimes \bar{\psi})=T_{1} \phi \otimes \overline{T_{2} \psi}
$$

the tensor product operator acting on $H_{n}^{2}\left(\dot{Z}^{\mathbf{C}}\right)$ for given operators $T_{1}, T_{2}$ acting on states $\phi, \psi \in H_{n}^{2}(\dot{Z})$. The Berezin symbol map, the dual Toeplitz map and the Berezin transform are invariant under $\dot{G}_{\mathbf{K}} \subset \dot{G}^{\mathbf{K}}$ acting on $L^{2}\left(\dot{Z}_{\mathbf{K}}, \dot{C}_{\mathbf{K}}^{\nu} \dot{\lambda}_{\mathbf{K}}\right)$ by substitution and on $H_{n}^{2}\left(\dot{Z}^{\mathbf{K}}\right)$ via the restriction of $\dot{U}_{n}^{\mathbf{K}}$ (which is a genuine (nonprojective) representation).

In the complex case we recover the classical concepts of Berezin-Toeplitz quantization. In the complex case we have $\kappa=2, d \lambda_{\mathbf{C}}(z, \bar{z})=d \lambda(z), \nu=n, \dot{C}_{\mathbf{C}}^{n}=\dot{C}_{n}$. It follows that

$$
d \dot{\lambda}_{\mathbf{C}}(z, \bar{z})=d \dot{\lambda}(z)
$$


is a $\dot{G}$-invariant measure on $\dot{Z}$. For the compact type we may take $n=0$, and

$$
\hat{C}_{0} d \hat{\lambda}_{\mathbf{C}}(z)=\hat{C}_{0} \hat{K}(z, z)^{-p} d \lambda(z)=\frac{\Gamma_{\Omega}(p)}{\pi^{d} \Gamma_{\Omega}\left(p-\frac{d}{r}\right)} \Delta(z,-z)^{-p} d \lambda(z)
$$

is the $\hat{G}$ invariant normalized Haar measure on $\hat{Z}$.

Definition 4.4. For a bounded operator $T$ on $H_{n}^{2}(\dot{Z})$ the Berezin operator symbol (evaluation on the normalized kernel functions) is defined by

$$
\left(\dot{S}_{n} T\right)(z):=\frac{\left(T \dot{K}_{z}^{n} \mid \dot{K}_{z}^{n}\right)}{\dot{K}_{n}(z, z)} .
$$

Specifically,

$$
\begin{gathered}
\left(S_{n} T\right)(z):=\frac{\left(T e_{z}^{n} \mid e_{z}^{n}\right)}{\exp (z \mid z)^{n}}, \\
\left(\check{S}_{n} T\right)(z):=\frac{\left(T \Delta_{z}^{-n} \mid \Delta_{z}^{-n}\right)}{\Delta(z, z)^{-n}}, \\
\left(\hat{S}_{n} T\right)(z):=\frac{\left(T \Delta_{-z}^{n} \mid \Delta_{-z}^{n}\right)}{\Delta(z,-z)^{n}}
\end{gathered}
$$

for operators $T$ on $H_{n}^{2}(\dot{Z})$.

Definition 4.5. For $f \in L^{\infty}(\dot{Z})$ the $n$-th Toeplitz operator acting on $H_{n}^{2}(\dot{Z})$ is defined by

$$
\left(\dot{T}_{n} f\right) \phi=\dot{P}_{n}(f \phi)
$$

for all $\phi \in H_{n}^{2}(\dot{Z})$.

In the compact case the $n$-th Toeplitz map $f \mapsto \hat{T}_{n} f$ is not injective, since $H_{n}^{2}(\hat{Z})$ has finite dimension, and it is necessary to consider the whole sequence.

Definition 4.6. The Berezin transform $f \mapsto \dot{B}_{n} f=\dot{S}_{n}\left(\dot{T}_{n} f\right)$ is an integral operator

$$
\left(\dot{B}_{n} f\right)(z)=\frac{\left(\left(\dot{T}_{n} f\right) \dot{K}_{z}^{n} \mid \dot{K}_{z}^{n}\right)}{\dot{K}_{n}(z, z)}=\frac{\left(f \dot{K}_{z}^{n} \mid \dot{K}_{z}^{n}\right)}{\dot{K}_{n}(z, z)}
$$

whose integral kernel with respect to (4.3) has the form

$$
\dot{B}_{n}(z, w)=\frac{\dot{K}_{n}(z, w) \dot{K}_{n}(w, z)}{\dot{K}_{n}(z, z) \dot{K}_{n}(w, w)}=\left(\frac{\dot{K}(z, w) \dot{K}(w, z)}{\dot{K}(z, z) \dot{K}(w, w)}\right)^{n} .
$$

Specifically,

$$
\begin{gathered}
B_{n}(z, w)=\left(\frac{\exp (z \mid w) \exp (w \mid z)}{\exp (z \mid z) \exp (w \mid w)}\right)^{n}, \\
\check{B}_{n}(z, w)=\left(\frac{\Delta(z, z) \Delta(w, w)}{\Delta(z, w) \Delta(w, z)}\right)^{n}, \\
\hat{B}_{n}(z, w)=\left(\frac{\Delta(z,-w) \Delta(w,-z)}{\Delta(z,-z) \Delta(w,-w)}\right)^{n} .
\end{gathered}
$$


In order to see the relationship with the concepts introduced above, note that any function $H(z, \bar{w}) \in H_{n}^{2}\left(\dot{Z}^{\mathbf{C}}\right)$ induces an integral operator

$$
(\tilde{H} \psi)(z)=\dot{C}_{n} \int_{\dot{Z}} d \dot{\lambda}(w) \dot{K}(w, w)^{-n} H(z, w) \psi(w)
$$

on $H_{n}^{2}(\dot{Z})$ of Hilbert-Schmidt type. Denoting the Hilbert-Schmidt operators by $\mathcal{L}^{2}$, the map $H \mapsto \tilde{H}$ provides an identification

$$
H_{n}^{2}\left(\dot{Z}^{\mathbf{C}}\right) \approx \mathcal{L}^{2}\left(H_{n}^{2}(\dot{Z})\right)
$$

For $\phi, \psi \in H_{n}^{2}(\dot{Z})$ the integral kernel $\phi \otimes \bar{\psi}$ corresponds to the rank 1 operator

$$
\widetilde{\phi \otimes \bar{\psi}}=\phi \psi^{*}
$$

as follows from the computation

$$
\begin{gathered}
\widetilde{\phi \otimes \bar{\psi}} h(z)=C_{n} \int_{\dot{Z}} d \dot{\lambda}(w) \dot{K}(w, w)^{-n}(\phi \otimes \bar{\psi})(z, w) h(w) \\
=C_{n} \int_{\dot{Z}} d \dot{\lambda}(w) \dot{K}(w, w)^{-n} \phi(z) \overline{\psi(w)} h(w) \\
=\phi(z) C_{n} \int_{\dot{Z}} d \dot{\lambda}(w) \dot{K}(w, w)^{-n} \overline{\psi(w)} h(w)=\phi(z)(h \mid \psi)=\left(\phi \psi^{*}\right) h(z),
\end{gathered}
$$

where $h \in H_{n}^{2}(\dot{Z})$.

\section{Proposition 4.7.}

$$
\left(T_{1} \widetilde{\otimes \bar{T}_{2}}\right) H=T_{1} \tilde{H} T_{2}^{*} .
$$

Proof. For rank 1 operators $\phi \psi^{*}$ with $\phi, \psi \in H_{n}^{2}(\dot{Z})$ we obtain for $h, k \in H_{n}^{2}(\dot{Z})$

$$
\begin{aligned}
\left(\phi_{1} \psi_{1}^{*} \otimes \overline{\phi_{2} \psi_{2}^{*}}\right)(h \otimes \bar{k}) & =\left(\phi_{1} \psi_{1}^{*} h\right) \otimes \overline{\phi_{2} \psi_{2}^{*} k} \\
=\left(\phi_{1}\left(h \mid \psi_{1}\right)\right) \otimes \overline{\phi_{2}\left(k \mid \psi_{2}\right)} & =\left(h \mid \psi_{1}\right)\left(\psi_{2} \mid k\right) \phi_{1} \otimes \overline{\phi_{2}} .
\end{aligned}
$$

It follows that

$$
\left(\phi_{1} \psi_{1}^{*} \otimes \widetilde{\overline{\phi_{2} \psi_{2}^{*}}}\right)(h \otimes \bar{k})=\left(\psi_{1} \mid h\right)\left(k \mid \psi_{2}\right) \widetilde{\phi_{1} \otimes \overline{\phi_{2}}}=\left(\psi_{1} \mid h\right)\left(k \mid \psi_{2}\right) \phi_{1} \phi_{2}^{*} .
$$

This agrees with

$$
\begin{aligned}
\left(\phi_{1} \psi_{1}^{*}\right)\left(\widetilde{(\overline{\operatorname{s} \bar{k}})}\left(\phi_{2} \psi_{2}^{*}\right)^{*}\right. & =\left(\phi_{1} \psi_{1}^{*}\right)\left(h k^{*}\right)\left(\psi_{2} \phi_{2}^{*}\right)=\phi_{1}\left(h \mid \psi_{1}\right)\left(\psi_{2} \mid k\right) \phi_{2}^{*} \\
& =\left(h \mid \psi_{1}\right)\left(\psi_{2} \mid k\right) \phi_{1} \phi_{2}^{*} .
\end{aligned}
$$

It follows from (4.2) and Proposition 4.7 that

$$
\dot{U}_{n}^{\mathbf{C}} \widetilde{\left(g_{1}, \bar{g}_{2}\right)} H=\left(\dot{U}_{n}\left(g_{1}\right) \widetilde{\dot{U}_{n}\left(g_{2}\right)}\right) H=\dot{U}_{n}\left(g_{1}\right) \tilde{H} \dot{U}_{n}\left(g_{2}\right)^{*}=\dot{U}_{n}\left(g_{1}\right) \tilde{H} \dot{U}_{n}\left(g_{2}^{-1}\right) \text {. }
$$

Thus, via the identification (4.6) the product action becomes the adjoint action

$$
\dot{U}_{n}^{\mathbf{C}}\left(g_{1}, \bar{g}_{2}\right) T=\dot{U}_{n}\left(g_{1}\right) T \dot{U}_{n}\left(g_{2}^{-1}\right)
$$

on operators.

\section{Proposition 4.8 .}

$$
\dot{\sigma}_{\mathbf{C}}^{n} H=\dot{S}_{n} \tilde{H}
$$


Proof. The Berezin symbol as defined in (4.1) is a densely defined map

$$
\dot{\sigma}_{\mathbf{C}}^{n}: H_{n}^{2}(\dot{Z}) \otimes \overline{H_{n}^{2}(\dot{Z})} \longrightarrow L^{2}\left(\dot{Z}, \dot{C}_{n} d \dot{\lambda}\right) .
$$

In view of (3.18) we obtain

$$
\dot{\sigma}_{\mathbf{C}}^{n}(\phi \otimes \bar{\psi})(z, \bar{z})=\frac{\phi(z) \overline{\psi(z)}}{\dot{K}_{n}^{\mathbf{C}}(z, \bar{z}, z, \bar{z})^{1 / 2}}=\frac{\phi(z) \overline{\psi(z)}}{\dot{K}_{n}(z, z)}
$$

for all $\phi, \psi \in H_{n}^{2}(\dot{Z})$. Therefore

$$
\widetilde{\phi \otimes \bar{\psi}} \dot{K}_{z}^{n}=\left(\phi \psi^{*}\right) \dot{K}_{z}^{n}=\phi\left(\dot{K}_{z}^{n} \mid \psi\right)=\phi \overline{\psi(z)}
$$

using (4.7) and the reproducing property (3.8), and hence

$$
\left.\widetilde{(\phi \otimes \bar{\psi}} \dot{K}_{z}^{n} \mid \dot{K}_{z}^{n}\right)=\left(\phi \overline{\psi(z)} \mid \dot{K}_{z}^{n}\right)=\left(\phi \mid \dot{K}_{z}^{n}\right) \overline{\psi(z)}=\phi(z) \overline{\psi(z)}
$$

using the reproducing property again. Comparing this with (4.4) it follows that

$$
\left(\dot{\sigma}_{\mathbf{C}}^{n} H\right)(z, \bar{z})=\frac{\left(\dot{K}_{z}^{n} \mid \tilde{H} \dot{K}_{z}^{n}\right)}{\dot{K}_{n}(z, z)}=\left(\dot{S}_{n} \tilde{H}\right)(z) .
$$

\section{Proposition 4.9.}

$$
\widetilde{\dot{\tau}_{\mathbf{C}}^{n} f}=\dot{T}_{n} f .
$$

Proof. By (9.8) and (3.18) the dual Toeplitz map

$$
\dot{\tau}_{\mathbf{C}}^{n}: L^{2}\left(\dot{Z}, \dot{C}_{n} d \dot{\lambda}\right) \longrightarrow H_{n}^{2}(\dot{Z}) \otimes \overline{H_{n}^{2}(\dot{Z})}
$$

has the form

$$
\begin{gathered}
\left(\dot{\tau}_{\mathbf{C}}^{n} f\right)\left(z_{1}, \bar{z}_{2}\right)=\dot{C}_{n} \int_{\dot{Z}_{\mathbf{C}}} d \dot{\lambda}_{\mathbf{C}}(w, \bar{w}) f(w) \frac{\dot{K}_{n}^{\mathbf{C}}\left(z_{1}, \bar{z}_{2}, w, \bar{w}\right)}{\dot{K}_{n}^{\mathbf{C}}(w, \bar{w}, w, \bar{w})^{1 / 2}} \\
=\dot{C}_{n} \int_{\dot{Z}} d \dot{\lambda}(w) f(w) \frac{\dot{K}_{n}\left(z_{1}, w\right) \dot{K}_{n}\left(w, z_{2}\right)}{\dot{K}_{n}(w, w)} \\
=\dot{C}_{n} \int_{\dot{Z}} d \dot{\lambda}(w) \dot{K}(w, w)^{-n} f(w) \dot{K}_{n}\left(z_{1}, w\right) \dot{K}_{n}\left(w, z_{2}\right) \\
=\dot{C}_{n} \int_{\dot{Z}} d \dot{\lambda}(w) f(w) \dot{K}_{n}\left(z_{1}, w\right) \dot{K}_{n}\left(w, z_{2}\right) \dot{K}(w, w)^{\epsilon p-n}=\dot{P}_{n}\left(f \dot{K}_{z_{2}}^{n}\right)\left(z_{1}\right)
\end{gathered}
$$

using (3.9). Therefore the associated integral operator is given by

$$
\begin{gathered}
\left(\widetilde{\dot{\tau}_{\mathbf{C}}^{n} f} \phi\right)(z)=\dot{C}_{n} \int_{\dot{Z}} d \dot{\lambda}(w) \dot{K}(w, w)^{-n}\left(\dot{\tau}_{\mathbf{C}}^{n} f\right)(z, \bar{w}) \phi(w) \\
=\dot{C}_{n} \int_{\dot{Z}} d \dot{\lambda}(w) \dot{K}(w, w)^{-n} \phi(w) \dot{P}_{n}\left(f \dot{K}_{w}^{n}\right)(z) \\
=\dot{P}_{n}\left(f \int_{\dot{Z}} d \dot{\lambda}(w) \dot{K}(w, w)^{-n} \phi(w) \dot{K}_{w}^{n}\right)(z)=\dot{P}_{n}(f \phi)(z) .
\end{gathered}
$$


Denote

$$
\dot{\beta}_{\mathbf{K}}(x, y)=\frac{\dot{K}^{\mathbf{K}}(x, y)}{\dot{K}^{\mathbf{K}}(x, x)^{1 / 2} \dot{K}^{\mathbf{K}}(y, y)^{1 / 2}}
$$

so that $\dot{\beta}_{\mathbf{K}}^{\nu}$ is an operator with integral kernel $\dot{\beta}_{\mathbf{K}}^{n}$ with respect to the measure $\dot{C}_{\mathbf{K}}^{\nu} \dot{\lambda}_{\mathbf{K}}$

Proposition 4.10.

$$
\dot{\beta}_{\mathbf{C}}^{n}(z, \bar{z}, w, \bar{w})=\dot{B}_{n}(z, w) .
$$

Proof.

$$
\begin{gathered}
\left(\dot{\beta}_{\mathbf{C}}^{n}\right)(z, \bar{z}, w, \bar{w})=\frac{\dot{K}_{n}^{\mathbf{C}}(z, \bar{z}, w, \bar{w})}{\dot{K}_{n}^{\mathbf{C}}(z, \bar{z}, z, \bar{z})^{1 / 2} \dot{K}_{n}^{\mathbf{C}}(w, \bar{w}, w, \bar{w})^{1 / 2}} \\
=\frac{\dot{K}_{n}(z, w) \dot{K}_{n}(w, z)}{\dot{K}_{n}(z, z) \dot{K}_{n}(w, w)}=\dot{B}_{n}(z, w) .
\end{gathered}
$$

\section{Asymptotic expansion for the Berezin transform}

We now turn to asymptotic expansions which are the main focus of the paper. Let $\nu$ be a scalar parameter. We write

$$
a_{\nu} \asymp b_{\nu} \quad \text { as } \nu \rightarrow \infty
$$

if $a_{\nu}-b_{\nu}=O\left(\nu^{-\infty}\right)$; i.e., for every $N \in \mathbf{N}$ the quantity $\nu^{N}\left(a_{\nu}-b_{\nu}\right)$ remains bounded. Similarly,

$$
a_{\nu} \asymp \sum_{j=0}^{\infty} \frac{b_{j}}{\nu^{j}} \quad \text { as } \nu \rightarrow \infty
$$

if for every $N \in \mathbf{N}$

$$
\left|a_{\nu}-\sum_{j=0}^{N-1} \frac{b_{j}}{\nu^{j}}\right|=O\left(\nu^{-N}\right) .
$$

Proposition 5.1. For all $z \in \dot{Z}$ we have

$$
\dot{K}(z, z) \geq 1
$$

with equality only for $z=0$, and

$$
\dot{K}(z, z)^{-1} \rightarrow 0
$$

for $\dot{Z} \ni z \rightarrow \dot{\infty}$. This notation means that in the compact or flat case the spectral norm $\|z\|_{\infty} \rightarrow \infty$, whereas in the bounded case $\|z\|_{\infty} \nearrow 1$.

Proof. In the flat case we have $\exp (z \mid z) \geq 1$, with equality only for $z=0$, and $\exp (z \mid z)^{-1} \rightarrow 0$ as $Z \ni z \rightarrow \infty$. In the other cases, a spectral decomposition argument shows $\Delta(z,-z) \geq 1$ for all $z \in Z$ and $\Delta(z, z) \leq 1$ for all $z \in \check{Z}$, in both cases with equality only for $z=0$. Moreover, $\Delta(z,-z)^{-1} \rightarrow 0$ as $Z \ni z \rightarrow \infty$ and $\Delta(z, z) \rightarrow 0$ for $z \in \check{Z}$ tending to the boundary.

Proposition 5.2. There exists a sesqui-holomorphic function $L(z, w)$ near $(0,0)$ of order $\geq 2$ in $z$ and $w$ such that

$$
\log \dot{K}(z, w)=(z \mid w)+\epsilon L(z, \epsilon w) .
$$


Proof. By $[\mathrm{L}$ there is an expansion

$$
\Delta(z, w)=1-(z \mid w)+h(z, w)=1-(z \mid w)+\sum_{j=2}^{r}(-1)^{j} h_{j}(z, w)
$$

for sesqui-polynomials $h_{j}$ which are bi-homogeneous of degree $(j, j)$. It follows that

$$
L(z, w):=-(z \mid w)-\log \Delta(z, w)=\sum_{n=2}^{\infty} \frac{((z \mid w)-h(z, w))^{n}}{n}-h(z, w)
$$

is a sesqui-holomorphic function near $(0,0)$ of order $\geq 2$ in $z$ and $w$. Since (3.2) implies

$$
\log \dot{K}(z, w)=\left(z \mid\left(1-\epsilon^{2}\right) w\right)-\epsilon \log \Delta(z, \epsilon w)=(z \mid w)+\epsilon L(z, \epsilon w),
$$

the assertion follows.

The asymptotic expansions will involve $\dot{G}_{\mathbf{K}^{-}}$-invariant differential operators on $\dot{Z}_{\mathbf{K}}$. For any $K_{\mathbf{K}}$-invariant polynomial $q$ on $Z_{\mathbf{K}}$ we denote by $q\left(\partial_{\mathbf{K}}\right)$ the associated constant coefficient differential operator on $Z_{\mathbf{K}}$. Since $q\left(\partial_{\mathbf{K}}\right)$ is $K_{\mathbf{K}}$-invariant, we can define a $\dot{G}_{\mathbf{K}}$-invariant differential operator $q\left(\dot{\partial}_{\mathbf{K}}\right)$ on $\dot{Z}_{\mathbf{K}}$ by

$$
\left(q\left(\dot{\partial}_{\mathbf{K}}\right) f\right)(x):=q\left(\partial_{\mathbf{K}}\right)\left(f \circ \dot{\mathfrak{t}}_{x}\right)(0)
$$

for all $x \in Z_{\mathbf{K}}$, where $\dot{\mathfrak{t}}_{x} \in \dot{G}_{\mathbf{K}}$ are the transvections (2.5). Equivalently,

$$
\left(q\left(\dot{\partial}_{\mathbf{K}}\right) f\right)(x)=q\left(\partial_{\mathbf{K}}\right)(f \circ g)(0),
$$

where $g \in \dot{G}_{\mathbf{K}}$ satisfies $g(0)=x$.

Theorem 5.3. For $j \in \mathbf{N}$ there exist unique $K_{\mathbf{K}}$-invariant real-valued polynomials $p_{j}$ on $Z_{\mathbf{K}}$, with associated $\dot{G}_{\mathbf{K}}$-invariant differential operators $p_{j}\left(\dot{\partial}_{\mathbf{K}}\right)$ on $\dot{Z}_{\mathbf{K}}$ as defined in (5.5), such that the Berezin transform has an asymptotic expansion as $\nu \rightarrow \infty$ :

$$
\left(\dot{\beta}_{\mathbf{K}}^{\nu} f\right)(x) \asymp \sum_{j=0}^{\infty} \frac{\left(p_{j}\left(\dot{\partial}_{\mathbf{K}}\right) f\right)(x)}{\nu^{j}}
$$

for all $f \in \mathcal{C}^{\infty}\left(\dot{Z}_{\mathbf{K}}\right)$ and $x \in \dot{Z}_{\mathbf{K}}$. Furthermore, $p_{0}=1$.

Proof. By invariance under $\dot{G}_{\mathbf{K}}$ it suffices to compute the Berezin transform at the origin:

Specifically,

$$
\begin{aligned}
& \left(\dot{\beta}_{\mathbf{K}}^{\nu} f\right)(0)=\dot{C}_{\mathbf{K}}^{\nu} \int_{\dot{Z}_{\mathbf{K}}} d \lambda_{\mathbf{K}}(x) \dot{K}(x, x)^{(\epsilon p-n) \kappa / 2} f(x) \\
= & \dot{C}_{\mathbf{K}}^{\nu} \int_{\dot{Z}_{\mathbf{K}}} d \lambda_{\mathbf{K}}(x) \dot{K}(x, x)^{-n \kappa / 2} \Delta(x, \epsilon x)^{-p \kappa / 2} f(x) .
\end{aligned}
$$

$$
\begin{gathered}
\left(\beta_{\mathbf{K}}^{\nu} f\right)(0)=C_{\mathbf{K}}^{\nu} \int_{Z_{\mathbf{K}}} d \lambda_{\mathbf{K}}(x) e^{-n \frac{\kappa}{2}(x \mid x)} f(x), \\
\left(\check{\beta}_{\mathbf{K}}^{\nu} f\right)(0)=\check{C}_{\mathbf{K}}^{\nu} \int_{\check{Z}_{\mathbf{K}}} d \lambda_{\mathbf{K}}(x) \Delta(x, x)^{(n-p) \kappa / 2} f(x), \\
\left(\hat{\beta}_{\mathbf{K}}^{\nu} f\right)(0)=\hat{C}_{\mathbf{K}}^{\nu} \int_{Z_{\mathbf{K}}} d \lambda_{\mathbf{K}}(x) \Delta(x,-x)^{-(n+p) \kappa / 2} f(x) .
\end{gathered}
$$

Using (5.1) and (5.2) it follows that

$$
\dot{K}^{\mathbf{K}}(z, z) \geq 1
$$


for all $z \in \dot{Z}^{\mathbf{K}}$, with equality only for $z=0$, and

$$
\dot{K}^{\mathbf{K}}(z, z)^{-1} \rightarrow 0
$$

for $\dot{Z}^{\mathbf{K}} \ni z \rightarrow \infty$. It follows that the real-valued "phase function"

$$
S(x):=-\frac{1}{2} \log \dot{K}^{\mathbf{K}}(x, x)
$$

on $\dot{Z}_{\mathbf{K}}$ satisfies $S(x) \leq 0$ for all $x \in \dot{Z}_{\mathbf{K}} \subset \dot{Z}^{\mathbf{K}}$, with equality only for $x=0$, and $S(x) \rightarrow-\infty$ for $\dot{Z}_{\mathbf{K}} \ni x \rightarrow \infty$. Using (5.4) we define

$$
L^{\mathbf{R}}(z, w):=L(z, w), \quad L^{\mathbf{C}}\left(z_{1}, \bar{z}_{2}, w_{1}, \bar{w}_{2}\right):=L\left(z_{1}, w_{1}\right)+L\left(w_{2}, z_{2}\right) .
$$

Then (5.3) implies

$$
\log \dot{K}^{\mathbf{K}}(z, w)=(z \mid w)^{\mathbf{K}}+\epsilon L^{\mathbf{K}}(z, \epsilon w)
$$

for all $z, w \in Z^{\mathbf{K}}$ near 0 . Hence

$$
-2 S(x)=(x \mid x)^{\mathbf{K}}+\epsilon L^{\mathbf{K}}(x, \epsilon x)
$$

for $x \in Z_{\mathbf{K}} \subset Z^{\mathbf{K}}$ near 0 , and $L^{\mathbf{K}}(x, \epsilon x)$ vanishes of order $\geq 4$ at 0 . Therefore $S(0)=0, S^{\prime}(0)=0$ and the second derivative $S^{\prime \prime}(0)$ corresponds to $-i d$ viewed as a real-valued quadratic form. Since its determinant is $\neq 0$ the unique critical point 0 of $S$ is non-degenerate. Thus the hypotheses of the stationary phase method (cf. [EU1, Theorem 3]) are satisfied. It follows that

$$
\int_{\dot{Z}_{\mathbf{K}}} d \lambda_{\mathbf{K}}(x) e^{n S(x)} \Delta(x, \epsilon x)^{-p \kappa / 2} f(x) \asymp \sum_{j=0}^{\infty} \frac{\left(R_{j}^{\prime} f\right)(0)}{\nu^{j+\kappa d / 2}}
$$

for uniquely determined $K_{\mathrm{K}}$-invariant differential operators $R_{j}^{\prime}$ independent of $\nu$. The normalization constants $C_{\mathbf{K}}^{\nu}$ are chosen such that

$$
\dot{C}_{\mathbf{K}}^{\nu} \int_{\dot{Z}_{\mathbf{K}}} d \dot{\lambda}_{\mathbf{K}}(x) K(x, x)^{-n \kappa / 2}=1
$$

or, equivalently,

$$
\left(\dot{\tau}_{\mathbf{K}}^{\nu} \mathbf{1}\right)(x)=\dot{K}(x, x)^{n \kappa / 2} .
$$

Therefore $\dot{\beta}_{\mathbf{K}}^{\nu} \mathbf{1}=\mathbf{1}$, and it follows as in the proof of [EU1, Proposition 1] that

$$
\left(\dot{\beta}_{\mathbf{K}}^{\nu} f\right)(0)=\dot{C}_{\mathbf{K}}^{\nu} \int_{\dot{Z}_{\mathbf{K}}} d \lambda_{\mathbf{K}}(x) e^{n S(x)} \Delta(x, \epsilon x)^{-p \kappa / 2} f(x) \asymp \sum_{j=0}^{\infty} \frac{\left(R_{j} f\right)(0)}{\nu^{j}}
$$

with $R_{0}=i d$. Write $R_{j}=p_{j}\left(\partial_{\mathbf{K}}\right)$ for uniquely determined $K_{\mathbf{K}}$ invariant polynomials $p_{j}$ on $Z_{\mathbf{K}}$. The assertion follows.

The asymptotic expansion (5.6) has the leading term $i d$ since $p_{0}=1$. Inverting this formal power series yields the asymptotic expansion as $\nu \rightarrow \infty$ :

$$
\left(\left(\dot{\beta}_{\mathbf{K}}^{\nu}\right)^{-1} f\right)(x) \asymp \sum_{j=0}^{\infty} \frac{\left(q_{j}\left(\dot{\partial}_{\mathbf{K}}\right) f\right)(x)}{\nu^{j}}
$$

of the inverse Berezin transform $\left(\dot{\beta}_{\mathbf{K}}^{\nu}\right)^{-1}$ in terms of unique $K_{\mathbf{K}}$-invariant real-valued polynomials $q_{j}$ on $Z_{\mathbf{K}}$. Unlike the Berezin transform, the inverse transform is not given by an integral operator and (5.9) holds only asymptotically. For the compact type the Berezin transform for fixed $\nu$ has finite rank and is not invertible. 
In the complex case, with $\nu=n$, we obtain the asymptotic expansion for the "operator" Berezin transform. Consider a $K$-invariant "real" sesqui-polynomial

$$
P(z, w)=\sum_{i} u_{i}(z) \overline{u_{i}(w)}
$$

Restricting to the diagonal we obtain a "real" differential operator

$$
P\left(\partial_{\mathbf{C}}\right):=\sum_{i} u_{i}(\partial) \overline{u_{i}(\partial)}
$$

on $Z_{\mathbf{C}}$. Here we define $\bar{D}$ for a differential operator $D$ by

$$
\bar{D} f=\overline{\overline{D f}} \text {. }
$$

Since $P\left(\partial_{\mathbf{C}}\right)$ is $K$-invariant, we can define a $\dot{G}$-invariant differential operator $P\left(\dot{\partial}_{\mathbf{C}}\right)$ by

$$
\left(P\left(\dot{\partial}_{\mathbf{C}}\right) f\right)(z)=P\left(\partial_{\mathbf{C}}\right)\left(f \circ \dot{\mathfrak{t}}_{z}\right)(0)
$$

for all $z \in \dot{Z}$. Here $\dot{\mathfrak{t}}_{z} \in \dot{G}$ are the transvections (2.5). Equivalently,

$$
\left(P\left(\dot{\partial}_{\mathbf{C}}\right) f\right)(z)=P\left(\partial_{\mathbf{C}}\right)(f \circ g)(0),
$$

where $g \in \dot{G}$ satisfies $g(0)=z$.

Proposition 5.4. For $j \in \mathbf{N}$ there exist unique $K$-invariant "real" sesqui-polynomials $P_{j}(z, w)$ such that the Berezin (operator) transform has an asymptotic expansion as $n \rightarrow \infty$ :

$$
\left(\dot{B}_{n} f\right)(z) \asymp \sum_{j=0}^{\infty} \frac{\left(P_{j}\left(\dot{\partial}_{\mathbf{C}}\right) f\right)(z)}{n^{j}}
$$

for $f \in \mathcal{C}^{\infty}(\dot{Z})$ and $z \in \dot{Z}$. Furthermore, $P_{0}=1$.

Proof. This follows from Theorem 5.3 for $\mathbf{K}=\mathbf{C}$ putting $P_{j}(z, z):=p_{j}(z, \bar{z})$. A direct proof as above is of course also possible.

The asymptotic expansion (5.12) has the leading term $i d$ corresponding to $P_{0}=$ 1. Inverting this formal power series yields the asymptotic expansion as $n \rightarrow \infty$ :

$$
\dot{B}_{n}^{-1} f(z) \asymp \sum_{j=0}^{\infty} \frac{\left(Q_{j}\left(\dot{\partial}_{\mathbf{C}}\right) f\right)(z)}{n^{j}}
$$

of the inverse operator Berezin transform $\dot{B}_{n}^{-1}$ in terms of uniquely determined $K$-invariant "real" sesqui-polynomials $Q_{j}$. Again, (5.13) holds only asymptotically. For the compact type the Berezin (operator) transform for fixed $n \in \mathbf{N}$ has finite rank and is not invertible.

\section{Asymptotic expansions of Wick And Anti-Wick type}

We will now introduce operator star-products associated with the ToeplitzBerezin quantization, including a generalization for real symmetric spaces. In this section we study their formal properties and establish rigorous definitions in terms of asymptotic expansions. More explicit "Peter-Weyl" formulas will be given in Section 10. Define a holomorphic function $\dot{I}_{\mathbf{K}}^{\nu}$ near $0 \in Z^{\mathbf{K}}$ by

$$
\dot{I}_{\mathbf{K}}^{\nu}(z)=\dot{K}_{n}^{\mathbf{K}}\left(z, z^{\sharp}\right)^{1 / 2} .
$$


Here we take the principal branch with $\dot{I}_{\mathbf{K}}^{\nu}(0)=1$. This function does not belong to $H_{n}^{2}\left(\dot{Z}^{\mathbf{K}}\right)$ but can be regarded as a multiplier.

Definition 6.1. The Berezin star-reduct (first syllable stressed)

$$
\dot{*}_{\nu}^{\mathbf{K}}: \mathcal{C}^{\infty}\left(\dot{Z}^{\mathbf{K}}\right) \longrightarrow \mathcal{C}^{\infty}\left(\dot{Z}_{\mathbf{K}}\right), \quad F \mapsto \dot{*}_{\nu}^{\mathbf{K}} F
$$

is formally determined by the requirement

$$
\overline{\dot{*}}_{\nu}^{\mathbf{K}}\left(\dot{S}_{n}^{\mathbf{K}} T\right)=\dot{\sigma}_{\mathbf{K}}^{\nu}\left(T \dot{I}_{\mathbf{K}}^{\nu}\right)
$$

for all operators $T$ on $H_{n}^{2}\left(\dot{Z}^{\mathbf{K}}\right)$ with the Berezin operator symbol $F=\dot{S}_{n}^{\mathbf{K}} T$.

Here $\dot{S}_{n}^{\mathbf{R}} T:=\dot{S}_{n} T$ and $\dot{S}_{n}^{\mathbf{C}}\left(T_{1} \otimes \overline{T_{2}}\right):=\left(\dot{S}_{n} T_{1}\right) \otimes\left(\dot{S}_{n} T_{2}^{*}\right)$ is the tensor product operator symbol defined by

$$
\begin{aligned}
& \left(\dot{S}_{n}^{\mathbf{C}}\left(T_{1} \otimes \overline{T_{2}}\right)\right)(z, w)=\left(\dot{S}_{n} T_{1}\right)(z) \overline{\left(\dot{S}_{n} T_{2}\right)(w)} \\
= & \left(\dot{S}_{n} T_{1}\right)(z)\left(\dot{S}_{n} T_{2}^{*}\right)(w)=\left(\dot{S}_{n} T_{1}\right) \otimes\left(\dot{S}_{n} T_{2}^{*}\right)(z, w)
\end{aligned}
$$

for operators $T_{1} \otimes \overline{T_{2}}$ acting on $H_{n}^{2}\left(\dot{Z}^{\mathbf{C}}\right)=H_{n}^{2}(\dot{Z}) \otimes \overline{H_{n}^{2}(\dot{Z})}$.

Definition 6.2. The Toeplitz star-reduct

$$
\dot{*}_{\nu}^{\mathbf{K}}: \mathcal{C}^{\infty}\left(\dot{Z}^{\mathbf{K}}\right) \longrightarrow \mathcal{C}^{\infty}\left(\dot{Z}_{\mathbf{K}}\right), \quad F \mapsto \dot{*}_{\nu}^{\mathbf{K}} F
$$

is formally determined by the requirement

$$
\dot{\tau}_{\mathbf{K}}^{\nu}\left(\dot{*}_{\nu}^{\mathbf{K}} F\right)=\left(\dot{T}_{n}^{\mathbf{K}} F\right) \dot{I}_{\mathbf{K}}^{\nu}
$$

for suitable functions $F$ on $\dot{Z}^{\mathbf{K}}$, with the Toeplitz operator $\dot{T}_{n}^{\mathbf{K}} F$ on $H_{n}^{2}\left(\dot{Z}^{\mathbf{K}}\right)$.

Here $\dot{T}_{n}^{\mathbf{R}} f:=\dot{T}_{n} f$ and $\dot{T}_{n}^{\mathbf{C}}\left(f_{1} \otimes f_{2}\right):=\left(\dot{T}_{n} f_{1}\right) \otimes \overline{\left(\dot{T}_{n} f_{2}\right)^{*}}$ is the tensor product Toeplitz operator on $H_{n}^{2}\left(\dot{Z}^{\mathbf{C}}\right)=H_{n}^{2}(\dot{Z}) \otimes \overline{H_{n}^{2}(\dot{Z})}$ given by

$$
\dot{T}_{n}^{\mathbf{C}}\left(f_{1} \otimes f_{2}\right)(\phi \otimes \bar{\psi})=\left(\left(\dot{T}_{n} f_{1}\right) \phi\right) \otimes \overline{\left(\dot{T}_{n} f_{2}\right)^{*} \psi} .
$$

\section{Proposition 6.3.}

$$
\dot{\beta}_{\mathbf{K}}^{\nu}\left(\dot{*}_{\nu}^{\mathbf{K}} F\right) \asymp \dot{\sigma}_{\mathbf{K}}^{\nu}\left(\left(\dot{T}_{n}^{\mathbf{K}} F\right) \dot{I}_{\mathbf{K}}^{\nu}\right) \asymp \bar{*}_{\mathbf{K}}^{\nu}\left(\dot{B}_{n}^{\mathbf{K}} F\right)
$$

for suitable functions $F$ on $\dot{Z}^{\mathbf{K}}$ with the Berezin operator transform $\dot{B}_{n}^{\mathbf{K}} F$.

Here $\dot{B}_{n}^{\mathbf{R}} f:=\dot{B}_{n} f$ and $\dot{B}_{n}^{\mathbf{C}}\left(f_{1} \otimes f_{2}\right):=\dot{S}_{n}^{\mathbf{C}} \dot{T}_{n}^{\mathbf{C}}\left(f_{1} \otimes f_{2}\right)=\left(\dot{B}_{n} f_{1}\right) \otimes\left(\dot{B}_{n} f_{2}\right)$.

Proof.

$$
\begin{gathered}
\dot{\beta}_{\mathbf{K}}^{\nu}\left(\dot{*}_{\nu}^{\mathbf{K}} F\right)=\dot{\sigma}_{\mathbf{K}}^{\nu} \dot{\tau}_{\mathbf{K}}^{\nu}\left(\dot{*}_{\nu}^{\mathbf{K}} F\right)=\dot{\sigma}_{\mathbf{K}}^{\nu}\left(\left(\dot{T}_{n}^{\mathbf{K}} F\right) \dot{I}_{\mathbf{K}}^{\nu}\right) \\
=\dot{*}_{\nu}^{\mathbf{K}}\left(\dot{S}_{n}^{\mathbf{K}}\left(\dot{T}_{n}^{\mathbf{K}} F\right)\right)=\dot{*}_{\nu}^{\mathbf{K}}\left(\dot{B}_{n}^{\mathbf{K}} F\right) .
\end{gathered}
$$

Up to now the operations (6.1) and (6.2) have been treated in a formal manner. For a rigorous definition in terms of asymptotic expansions we start with the balanced operation

$$
F \mapsto \dot{\sigma}_{\mathbf{K}}^{\nu}\left(\left(\dot{T}_{n}^{\mathbf{K}} F\right) \dot{I}_{\mathbf{K}}^{\nu}\right)
$$

from $\dot{Z}^{\mathbf{K}}$ to $\dot{Z}_{\mathbf{K}}$ which is rigorously defined as an integral operator:

$$
\dot{\sigma}_{\mathbf{K}}^{\nu}\left(\left(\dot{T}_{n}^{\mathbf{K}} F\right) \dot{I}_{\mathbf{K}}^{\nu}\right)(x)=\dot{C}_{n}^{\kappa} \int_{\dot{Z}^{\mathbf{K}}} d \dot{\lambda}^{\mathbf{K}}(w) \frac{\dot{K}_{n}^{\mathbf{K}}(x, w) \dot{K}_{n}^{\mathbf{K}}\left(w, w^{\sharp}\right)^{1 / 2}}{\dot{K}_{n}^{\mathbf{K}}(w, w) \dot{K}_{n}^{\mathbf{K}}(x, x)^{1 / 2}} F(w) .
$$


This follows from the computation

$$
\begin{gathered}
\dot{\sigma}_{\mathbf{K}}^{\nu}\left(\left(\dot{T}_{n}^{\mathbf{K}} F\right) \dot{I}_{\mathbf{K}}^{\nu}\right)(x)=\frac{\left(\left(\dot{T}_{n}^{\mathbf{K}} F\right) \dot{I}_{\mathbf{K}}^{\nu}\right)(x)}{\dot{I}_{\mathbf{K}}^{\nu}(x)}=\frac{\dot{P}_{n}^{\mathbf{K}}\left(F \dot{I}_{\mathbf{K}}^{\nu}\right)(x)}{\dot{I}_{\mathbf{K}}^{\nu}(x)} \\
=\dot{C}_{n}^{\kappa} \int_{\dot{Z}^{\mathbf{K}}} d \dot{\lambda}^{\mathbf{K}}(w) \dot{K}^{\mathbf{K}}(w, w)^{-n} \frac{\dot{K}_{n}^{\mathbf{K}}(x, w) \dot{I}_{\mathbf{K}}^{\nu}(w)}{\dot{I}_{\mathbf{K}}^{\nu}(x)} F(w) \\
=\dot{C}_{n}^{\kappa} \int_{\dot{Z}^{\mathbf{K}}} d \dot{\lambda}^{\mathbf{K}}(w) \frac{\dot{K}_{n}^{\mathbf{K}}(x, w) \dot{K}_{n}^{\mathbf{K}}\left(w, w^{\sharp}\right)^{1 / 2}}{\dot{K}_{n}^{\mathbf{K}}(w, w) \dot{K}_{n}^{\mathbf{K}}(x, x)^{1 / 2}} F(w) .
\end{gathered}
$$

Here the orthogonal projection $\dot{P}_{n}^{\mathbf{K}}$ onto $H_{n}^{2}\left(\dot{Z}^{\mathbf{K}}\right)$ is given by

$$
\dot{P}_{n}^{\mathbf{R}}:=\dot{P}_{n}, \quad \dot{P}_{n}^{\mathbf{C}}:=\dot{P}_{n} \otimes \dot{P}_{n} .
$$

We will now derive an asymptotic expansion for the balanced operation.

\section{Proposition 6.4.}

$$
|\dot{K}(z, w)| \leq \dot{K}(z, z)^{1 / 2} \dot{K}(w, w)^{1 / 2} .
$$

Proof. The Cauchy-Schwarz inequality in $H_{n}^{2}(\dot{Z})$ implies

$$
\left|\dot{K}_{n}(z, w)\right|=\left|\left(\dot{K}_{z}^{n} \mid \dot{K}_{w}^{n}\right)\right| \leq\left(\dot{K}_{z}^{n} \mid \dot{K}_{z}^{n}\right)^{1 / 2}\left(\dot{K}_{w}^{n} \mid \dot{K}_{w}^{n}\right)^{1 / 2}=\dot{K}_{n}(z, z)^{1 / 2} \dot{K}_{n}(w, w)^{1 / 2} .
$$

Proposition 6.5. For $j \in \mathbf{N}$ there exist differential operators $\mathcal{M}_{j}$ on a neighborhood of $\dot{Z}_{\mathbf{K}} \subset \dot{Z}^{\mathbf{K}}$ which are invariant under $\dot{G}_{\mathbf{K}} \subset \dot{G}^{\mathbf{K}}$ and yield an asymptotic expansion as $\nu \rightarrow+\infty$ :

$$
\dot{\sigma}_{\mathbf{K}}^{\nu}\left(\left(\dot{T}_{n}^{\mathbf{K}} F\right) \dot{I}_{\mathbf{K}}^{\nu}\right)(x) \asymp \sum_{j=0}^{\infty} \frac{\left(\mathcal{M}_{j} F\right)(x)}{\nu^{j}}
$$

for $F \in \mathcal{C}^{\infty}\left(\dot{Z}^{\mathbf{K}}\right)$ and $x \in \dot{Z}_{\mathbf{K}}$. The operators $\mathcal{M}_{j}$ are not uniquely determined, but their restriction to $\dot{Z}_{\mathbf{K}}$ is. Moreover, $\mathcal{M}_{0}=i d$ is the identity map.

Proof. By $\dot{G}_{\mathbf{K}}$-invariance, it suffices to evaluate the integral (6.5) at $x=0$. Using (6.6) we obtain

$$
\begin{aligned}
\dot{\sigma}_{\mathbf{K}}^{\nu}\left(\left(\dot{T}_{n}^{\mathbf{K}} F\right) \dot{I}_{\mathbf{K}}^{\nu}\right)(0) & =\dot{P}_{n}^{\mathbf{K}}\left(F \dot{I}_{\mathbf{K}}^{\nu}\right)(0)=\dot{C}_{n}^{\kappa} \int_{\dot{Z}^{\mathbf{K}}} d \lambda^{\mathbf{K}}(z) \dot{K}^{\mathbf{K}}(z, z)^{\epsilon p-n} F(z) \dot{I}_{\mathbf{K}}^{\nu}(z) \\
= & \dot{C}_{n}^{\kappa} \int_{\dot{Z}^{\mathbf{K}}} d \lambda^{\mathbf{K}}(z) \dot{K}^{\mathbf{K}}(z, z)^{\epsilon p-n} \dot{K}^{\mathbf{K}}\left(z, z^{\sharp}\right)^{n / 2} F(z) \\
= & \dot{C}_{n}^{\kappa} \int_{\dot{Z}^{\mathbf{K}}} d \lambda^{\mathbf{K}}(z) \dot{K}^{\mathbf{K}}(z, z)^{\epsilon p}\left(\frac{\dot{K}^{\mathbf{K}}\left(z, z^{\sharp}\right)^{1 / 2}}{\dot{K}^{\mathbf{K}}(z, z)}\right)^{n} F(z) .
\end{aligned}
$$

Applying (6.7) we obtain

$$
\left|\dot{K}_{n}^{\mathbf{K}}(z, w)\right| \leq \dot{K}_{n}^{\mathbf{K}}(z, z)^{1 / 2} \dot{K}_{n}^{\mathbf{K}}(w, w)^{1 / 2}
$$

for all $z, w \in \dot{Z}^{\mathrm{K}}$. In particular,

$$
\left|\dot{K}_{n}^{\mathbf{K}}\left(w, w^{\sharp}\right)\right| \leq \dot{K}_{n}^{\mathbf{K}}(w, w)^{1 / 2} \dot{K}_{n}^{\mathbf{K}}\left(w^{\sharp}, w^{\sharp}\right)^{1 / 2}=\dot{K}_{n}^{\mathbf{K}}(w, w),
$$

and hence

$$
\frac{\left|\dot{K}^{\mathbf{K}}\left(z, z^{\sharp}\right)^{1 / 2}\right|}{\dot{K}^{\mathbf{K}}(z, z)} \leq \dot{K}^{\mathbf{K}}(z, z)^{-1 / 2} .
$$


It follows that the complex-valued "phase function"

$$
S(z)=\log \frac{\dot{K}^{\mathbf{K}}\left(z, z^{\sharp}\right)^{1 / 2}}{\dot{K}^{\mathbf{K}}(z, z)}
$$

satisfies $\operatorname{Re} S(z) \leq 0$ for all $z \in \dot{Z}^{\mathbf{K}}$, with equality only for $z=0$, and also $\operatorname{Re} S(z) \rightarrow$ $-\infty$ as $\dot{Z}^{\mathbf{K}} \ni z \rightarrow \infty$. Using (5.7) we have

$$
S(z)=\frac{1}{2} \log \dot{K}^{\mathbf{K}}\left(z, z^{\sharp}\right)-\log \dot{K}^{\mathbf{K}}(z, z)=\left(z \mid \frac{z^{\sharp}}{2}-z\right)^{\mathbf{K}}+\frac{\epsilon}{2} L^{\mathbf{K}}\left(z, \epsilon z^{\sharp}\right)-\epsilon L^{\mathbf{K}}(z, \epsilon z) .
$$

Thus $S(0)=0, S^{\prime}(0)=0$ and the second derivative,

$$
S^{\prime \prime}(0)(x+i y)=\left(x+i y \mid \frac{x-i y}{2}-x-i y\right)^{\mathbf{K}}=-\frac{1}{2}(x \mid x)^{\mathbf{K}}-\frac{3}{2}(y \mid y)^{\mathbf{K}}+i(x \mid y)^{\mathbf{K}},
$$

regarded as a $\mathbf{C}$-valued quadratic form in $(x, y)$, with $x, y \in Z_{\mathbf{K}}$, corresponds to the block-matrix

$$
\left(\begin{array}{cc}
-1 & i \\
i & -3
\end{array}\right)
$$

Since its determinant is $\neq 0$, the unique critical point $0 \in \dot{Z}^{\mathbf{K}}$ of $S$ is non-degenerate. Thus the hypotheses of the stationary phase method (cf. [EU1, Theorem 3]) are satisfied. It follows that there is an asymptotic expansion

$$
\int_{\dot{Z}^{\mathbf{K}}} d \lambda^{\mathbf{K}}(z) \dot{K}^{\mathbf{K}}(z, z)^{\epsilon p-n} \dot{K}^{\mathbf{K}}\left(z, z^{\sharp}\right)^{n / 2} F(z) \asymp n^{-\kappa d} \sum_{j=0}^{\infty} \frac{M_{j}^{\prime} F(0)}{\nu^{j}}
$$

as $\nu \rightarrow \infty$, where $M_{j}^{\prime}$ are unique $K_{\mathbf{K}}$-invariant differential operators independent of $\nu$. Passing to the normalized operators $M_{j}$ as in the proof for the Berezin transform, the assertion follows.

In view of (6.3) we give rigorous definitions of (‥1) and (6.2) in terms of asymptotic expansions.

Definition 6.6. The Berezin star-reduct $\dot{\dot{*}}_{\nu}^{\mathbf{K}}$ is the asymptotic expansion as $\nu \rightarrow \infty$ :

$$
\left(\overline{\dot{*}}_{\nu}^{\mathbf{K}} F\right)(x) \asymp \sum_{j=0}^{\infty} \frac{\left(\mathcal{N}_{j} F\right)(x)}{\nu^{j}}
$$

for unique differential operators $\mathcal{N}_{j}$ from $\dot{Z}^{\mathbf{K}}$ to $\dot{Z}_{\mathbf{K}}$ invariant under $\dot{G}_{\mathbf{K}} \subset \dot{G}^{\mathbf{K}}$, which is obtained by composing (6.8) from the right with the asymptotic expansion of the inverse operator Berezin transform $\left(\dot{B}_{n}^{\mathbf{K}}\right)^{-1}$.

Here $\left(\dot{B}_{n}^{\mathbf{R}}\right)^{-1}:=\dot{B}_{n}^{-1}$ and $\left(\dot{B}_{n}^{\mathbf{C}}\right)^{-1}=\dot{B}_{n}^{-1} \otimes \dot{B}_{n}^{-1}$ is the tensor product asymptotic expansion.

Definition 6.7. The Toeplitz star-reduct $\dot{*}_{\nu}^{\mathbf{K}} F$ is the asymptotic expansion as $\nu \rightarrow$ $\infty$ :

$$
\left(\dot{*}_{\nu}^{\mathbf{K}} F\right)(x) \asymp \sum_{j=0}^{\infty} \frac{\left(\mathcal{L}_{j} F\right)(x)}{\nu^{j}},
$$

for unique differential operators $\mathcal{L}_{j}$ from $\dot{Z}^{\mathbf{K}}$ to $\dot{Z}_{\mathbf{K}}$ invariant under $\dot{G}_{\mathbf{K}} \subset \dot{G}^{\mathbf{K}}$, which is obtained by composing the asymptotic expansion (6.8) from the left with the asymptotic expansion (5.9) of the inverse Berezin transform $\left(\dot{\beta}_{\mathbf{K}}^{\nu}\right)^{-1}$. 
The complex case, with $\nu=n$, yields the familiar star-products, which for the flat type (Fock space) correspond to the (anti-) Wick calculus. Since (3.18) implies

$$
\dot{I}_{\mathbf{C}}^{n}(z, \bar{w})=\dot{K}_{n}^{\mathbf{C}}(z, \bar{w}, w, \bar{z})^{1 / 2}=\dot{K}_{n}(z, w),
$$

the associated integral operator is

$$
\tilde{\dot{I}}_{n}^{\mathbf{C}}=i d \text {. }
$$

Definition 6.8. The Berezin star-product $f_{1} \underset{n}{\dot{*}} f_{2}$ is formally defined by

$$
\left(\dot{S}_{n} T_{1}\right) \underset{n}{\dot{*}}\left(\dot{S}_{n} T_{2}\right)=\dot{S}_{n}\left(T_{1} T_{2}\right)
$$

for operators $T_{i}$ on $H_{n}^{2}(\dot{Z})$.

In the non-compact setting, where $S_{n}$ is one-to-one, this determines $\overline{\dot{*}}$ unambiguously; in the compact case the Berezin star-product will again be defined by an asymptotic expansion.

Definition 6.9. For $f_{1}, f_{2} \in \mathcal{C}^{\infty}(\dot{Z})$ the Toeplitz star-product $f_{1} \dot{*} f_{2}$ is formally defined by

$$
\dot{T}_{n}\left(f_{1} \dot{*} f_{2}\right)=\left(\dot{T}_{n} f_{1}\right)\left(\dot{T}_{n} f_{2}\right)
$$

\section{Proposition 6.10.}

$$
\dot{*}_{\mathbf{C}}^{n}\left(f_{1} \otimes f_{2}\right)=f_{1} \dot{*} f_{2} .
$$

Proof. For $H \in H_{n}^{2}(\dot{Z})$ we regard $\left(T_{1} \otimes \bar{T}_{2}\right) H \in H_{n}^{2}(\dot{Z})$ as an integral kernel. Combining (6.13) and (4.8) we obtain

$$
\dot{T}_{n}^{\mathbf{C}}\left(\widetilde{f_{1} \otimes f_{2}}\right) H=\left(\left(\dot{T}_{n} f_{1}\right) \widetilde{\otimes\left(\dot{T}_{n} f_{2}\right)^{*}}\right) H=\left(\dot{T}_{n} f_{1}\right) \tilde{H}\left(\dot{T}_{n} f_{2}\right) .
$$

Thus

$$
\begin{aligned}
& \dot{\tau}_{\mathbf{C}}^{n}\left(\dot{*}_{\mathbf{C}}^{n}\left(f_{1} \otimes f_{2}\right)\right)=\dot{T}_{n}^{\mathbf{C}}\left(\widetilde{f_{1} \otimes f_{2}}\right) \dot{I}_{\mathbf{C}}^{n}=\left(\dot{T}_{n} f_{1}\right) \widetilde{\widetilde{\left(\dot{T}_{n} f_{2}\right)^{*}}} \dot{I}_{\mathbf{C}}^{n} \\
= & \left.\left(\dot{T}_{n} f_{1}\right) \widetilde{\dot{I}_{\mathbf{C}}^{n}}\left(\dot{T}_{n} f_{2}\right)=\left(\dot{T}_{n} f_{1}\right)\left(\dot{T}_{n} f_{2}\right)=\dot{T}_{n}\left(f_{1} \dot{*} f_{2}\right)=\dot{\tau}_{\mathbf{C}}^{n} \widetilde{\left(f_{1} \dot{*} f_{2}\right.}\right),
\end{aligned}
$$

yielding the assertion. Only in the non-compact case is the Toeplitz map $f \mapsto T_{n} f$ injective. For the compact type only the whole sequence $\dot{T}_{n},(n \in \mathbf{N})$ is injective and (6.14) is an identity of asymptotic expansions.

\section{Proposition 6.11.}

$$
\overline{\dot{*}}_{\mathbf{C}}^{n}\left(f_{1} \otimes f_{2}\right)=f_{1_{n}} \overline{\dot{*}} f_{2} .
$$

Proof. For operators $T_{1}, T_{2}$ on $H_{n}^{2}(\dot{Z})$ we have

$$
\begin{aligned}
& {\overline{\dot{*}^{\prime}}}_{\mathbf{C}}^{n}\left(\left(\dot{S}_{n} T_{1}\right) \otimes\left(\dot{S}_{n} T_{2}\right)\right)=\overline{\dot{*}}_{\mathbf{C}}^{n}\left(\left(\dot{S}_{n} T_{1}\right) \otimes \overline{\dot{S}_{n} T_{2}^{*}}\right)=\overline{\dot{*}}_{\mathbf{C}}^{n}\left(\dot{S}_{n}^{\mathbf{C}}\left(T_{1} \otimes \overline{T_{2}^{*}}\right)\right) \\
& =\dot{\sigma}_{\mathbf{C}}^{n}\left(\left(T_{1} \otimes \overline{T_{2}^{*}}\right) \dot{I}_{\mathbf{C}}^{n}\right)=\dot{S}_{n}\left(T_{1} \widetilde{\otimes T_{2}^{*}}\right) \dot{I}_{\mathbf{C}}^{n}=\dot{S}_{n}\left(T_{1} \widetilde{\dot{I}_{\mathbf{C}}^{n}} T_{2}\right)=\dot{S}_{n}\left(T_{1} T_{2}\right)=\left(\dot{S}_{n} T_{1}\right) \underset{n}{\dot{*}}\left(\dot{S}_{n} T_{2}\right) \text {. }
\end{aligned}
$$

\section{Proposition 6.12.}

$$
\dot{B}_{n}\left(f_{1} \dot{*} f_{2}\right)=\dot{S}_{n} \dot{T}_{n}\left(f_{1} \dot{*} f_{2}\right)=\dot{S}_{n}\left[\left(\dot{T}_{n} f_{1}\right)\left(\dot{T}_{n} f_{2}\right)\right]=\left(\dot{B}_{n} f_{1}\right) \underset{n}{\dot{*}}\left(\dot{B}_{n} f_{2}\right) .
$$


Proof. Putting $T_{1}=\dot{T}_{n} f_{1}, T_{2}=\dot{T}_{n} f_{2}$ and recalling (6.12), the assertion follows with 6.13).

The balanced operation (6.4) corresponds to the (non-associative) product operation

$$
f_{1} \otimes f_{2} \mapsto \dot{S}_{n}\left[\left(\dot{T}_{n} f_{1}\right)\left(\dot{T}_{n} f_{2}\right)\right](z)
$$

which has the integral kernel

$$
\frac{\dot{K}_{n}\left(z, w_{1}\right) \dot{K}_{n}\left(w_{1}, w_{2}\right) \dot{K}_{n}\left(w_{2}, z\right)}{\dot{K}_{n}\left(w_{1}, w_{1}\right) \dot{K}_{n}(z, z) \dot{K}_{n}\left(w_{2}, w_{2}\right)}
$$

with respect to (4.3).

Proposition 6.13. There exist bi-differential operators $\mathcal{M}_{j}$ on $\dot{Z} \times \overline{\dot{Z}}$ which are invariant under $\dot{G} \subset \dot{G} \times \overline{\dot{G}}$ and yield an asymptotic expansion as $n \rightarrow+\infty$ :

$$
\dot{S}_{n}\left(\left(\dot{T}_{n} f_{1}\right)\left(\dot{T}_{n} f_{2}\right)\right) \asymp \sum_{j=0}^{\infty} \frac{\mathcal{M}_{j}\left(f_{1} \otimes f_{2}\right)}{n^{j}}
$$

for $f_{1}, f_{2} \in \mathcal{C}^{\infty}(\dot{Z})$. Moreover, $\mathcal{M}_{0}\left(f_{1} \otimes f_{2}\right)=f_{1} f_{2}$ is the product map.

Proof. By $\dot{G}$-invariance, we may assume $z=0$ (although $f_{1} \otimes f_{2}$ depends on $(z, w)$ and the invariance is only with respect to the diagonal action $f_{1} \otimes f_{2} \mapsto\left(f_{1} \circ g\right) \otimes$ $\left(f_{2} \circ g\right)$ of $\left.g \in \dot{G}\right)$. Then

$$
\begin{aligned}
& \dot{S}_{n}\left[\left(\dot{T}_{n} f_{1}\right)\left(\dot{T}_{n} f_{2}\right)\right](0)=\left(1 \mid\left(\dot{T}_{n} f_{1}\right)\left(\dot{T}_{n} f_{2}\right) 1\right)=\left(1 \mid f_{1} \cdot \dot{P}_{n} f_{2}\right) \\
& =\dot{C}_{n} \int_{\dot{Z}} d \dot{\lambda}(z) \dot{K}(z, z)^{-n} f_{1}(z)\left(\dot{P}_{n} f_{2}\right)(z) \\
& =\dot{C}_{n}^{2} \int_{\dot{Z}} d \dot{\lambda}(z) \dot{K}(z, z)^{-n} f_{1}(z) \int_{\dot{Z}} d \dot{\lambda}(w) \dot{K}(w, w)^{-n} \dot{K}_{n}(z, w) f_{2}(w) \\
& =\dot{C}_{n}^{2} \int_{\dot{Z}^{\mathbf{C}}} d \dot{\lambda}^{\mathbf{C}}(z, w) \dot{K}(z, z)^{-n} \dot{K}(w, w)^{-n} \dot{K}_{n}(z, w) f_{1}(z) f_{2}(w) \\
& =\dot{C}_{n}^{2} \int_{\dot{Z}^{\mathbf{C}}} d \lambda^{\mathbf{C}}(z, w) \frac{\dot{K}(z, w)^{n}}{\dot{K}(z, z)^{n+p} \dot{K}(w, w)^{n+p}} f_{1}(z) f_{2}(w) .
\end{aligned}
$$

In the compact setting $\hat{K}(z, w)=\Delta(z,-w)$ can have zeros but we may restrict the integration to the open dense subset $\Omega:=\left\{(z, w) \in Z \times Z: \Delta(z,-w) \in \mathbf{C} \backslash \mathbf{R}_{-}\right\}$of $\hat{Z} \times \hat{Z}$ which has full Lebesgue measure being the complement of a real semi-algebraic subset with Lebesgue measure 0. Define the complex-valued "phase function"

$$
S(z, w):=\log \frac{\dot{K}(z, w)}{\dot{K}(z, z) \dot{K}(w, w)}
$$

on $\dot{Z}^{\mathbf{C}}$ (or $\Omega \subset Z^{\mathbf{C}}$ ), using the principal branch of the logarithm. By (6.7) we have

$$
\operatorname{Re} S(z, w)=\log \frac{|\dot{K}(z, w)|}{\dot{K}(z, z) \dot{K}(w, w)} \leq \log \dot{K}(z, z)^{-1 / 2} \dot{K}(w, w)^{-1 / 2} \leq 0,
$$

since $\dot{K}(z, z) \geq 1$ for all $z \in \dot{Z}$. The same estimate shows $\operatorname{Re} S(z, w) \rightarrow-\infty$ for $\dot{Z}^{\mathrm{C}} \ni(z, w) \rightarrow \infty$. Using the expansion

$$
S(z, w)=(z \mid w)-(z \mid z)-(w \mid w)+\epsilon(L(z, \epsilon w)-L(z, \epsilon z)-L(w, \epsilon w))
$$


for $z, w$ near $0 \in Z$ as in (5.7), it follows that $S(0,0)=0, S^{\prime}(0,0)=0$ and the second derivative $S^{\prime \prime}(0,0)$ corresponds to the complex-valued quadratic form

$$
(z \mid w)-(z \mid z)-(w \mid w)=-\left((z, \bar{w}) \mid(z, \bar{w})-\frac{1}{2}(w, \bar{z})\right)^{\mathbf{C}} .
$$

Since the associated complex-valued quadratic form has determinant $\neq 0$ the unique critical point $(0,0)$ of $S$ is non-degenerate. Thus the hypotheses of the stationary phase method (cf. EU1, Theorem 3]) are satisfied. It follows that there is an asymptotic expansion

$$
\int_{\Omega} d \lambda(z, w) \frac{\dot{K}(z, w)^{n}}{\dot{K}(z, z)^{n+p} \dot{K}(w, w)^{n+p}} f_{1}(z) f_{2}(w) \asymp n^{-2 d} \sum_{j=0}^{\infty} \frac{M_{j}^{\prime}\left(f_{1} \otimes f_{2}\right)(0,0)}{n^{j}}
$$

for $n \rightarrow \infty$, where $M_{j}^{\prime}$ are unique $K$-invariant bi-differential operators independent of $n$. Now we pass to the normalized operators $M_{j}$ and put

$$
\mathcal{M}_{j}\left(f_{1} \otimes f_{2}\right)(z):=M_{j}\left(\left(f_{1} \circ \dot{\mathfrak{t}}_{z}\right) \otimes\left(f_{2} \circ \dot{\mathfrak{t}}_{z}\right)\right)(0,0) .
$$

As above, we use (6.15) to give rigorous definitions of the operator star-products. The Toeplitz star-product is defined as the asymptotic expansion

$$
f_{1} \dot{*} f_{2} \asymp \sum_{j=0}^{\infty} \frac{\mathcal{L}_{j}\left(f_{1} \otimes f_{2}\right)}{n^{j}}
$$

obtained as a composition of two formal power series in $n^{-1}$ with operator coefficients, namely the inverse of $\dot{B}_{n}$ and the asymptotic expansion (6.15). Equivalently,

$$
\left(\dot{T}_{n} f_{1}\right)\left(\dot{T}_{n} f_{2}\right) \asymp \sum_{j=0}^{\infty} \frac{\dot{T}_{n}\left(\mathcal{L}_{j}\left(f_{1} \otimes f_{2}\right)\right)}{n^{j}} .
$$

It is the main result of $\mathrm{Sch}$ that this expansion in fact holds (even on an arbitrary compact Kähler manifold) in the much stronger sense of operator norms:

$$
\left\|\left(\dot{T}_{n} f_{1}\right)\left(\dot{T}_{n} f_{2}\right)-\sum_{j=0}^{N-1} \frac{\dot{T}_{n}\left(\mathcal{L}_{j}\left(f_{1} \otimes f_{2}\right)\right)}{n^{j}}\right\|=O\left(n^{-N}\right) \quad \text { as } n \rightarrow+\infty
$$

for any $N \in \mathbf{N}$. (Similar results also hold in the non-compact case BLU], E2].) This stronger version is not needed for our results, but will be used later in an alternative proof.

The dual Berezin star-product is defined as the asymptotic expansion

$$
f_{1} \underset{n}{\bar{*}} f_{2} \asymp \sum_{j=0}^{\infty} \frac{\mathcal{N}_{j}\left(f_{1} \otimes f_{2}\right)}{n^{j}}
$$

obtained as the conjugate

$$
f_{1} \underset{n}{\dot{*}} f_{2}:=\dot{B}_{n}\left[\left(\dot{B}_{n}^{-1} f_{1}\right) \underset{n}{\dot{*}}\left(\dot{B}_{n}^{-1} f_{2}\right)\right]
$$

of the Toeplitz star-product $\underset{n}{*}$ by the Berezin transform $\dot{B}_{n}$ (viewed as a formal power series in $\frac{1}{n}$ ). This also applies for the compact type which up to now was only treated in a formal manner. 


\section{THE LOCAL MODEL}

A crucial step in the proof of the "factorization properties" below is to assign a socalled local model to $\dot{Z}$. This is of particular importance for the compact type which has only constant holomorphic functions, and to a lesser extent for the flat case which has only constant bounded holomorphic functions. In the general situation it is still instructive to consider smooth local models in order to make contact with the extensive literature concerning Toeplitz operators on smooth domains, and also because concepts associated with the local model are only invariant under the compact group $K$. This may be useful to treat more general quantization procedures having only $K$-invariance.

Consider the unit ball

$$
\underset{\sim}{Z}=\{z \in Z:\|z\|<1\} \subset Z
$$

for $\|z\|:=\sqrt{(z \mid z)}$. For $r>1$ this norm is different from the spectral norm, but we have

$$
\underset{\sim}{Z} \subset \check{Z} \subset Z \subset \hat{Z} .
$$

Proposition 7.1. There exists a smooth defining function $\dot{W}$ on the closure of $\underset{\sim}{Z}$ such that

$$
\begin{gathered}
\dot{W}>0,-\log \dot{W} \text { strictly plurisubharmonic on } \underset{\sim}{Z}, \\
\dot{W}=0, \nabla \dot{W} \neq 0 \text { on the boundary } \partial \underset{\sim}{Z}, \\
\dot{W}(z)=\dot{K}(z, z)^{-1} \text { for }\|z\| \leq 1 / 2 .
\end{gathered}
$$

Proof. This is shown as in the proof of Lemma 6 in E1].

Endowed with the defining function $\dot{W}$ we call $\underset{\sim}{Z}$ the local model of $\dot{Z}$ and denote it by $\underset{\sim}{\dot{Z}}$. Consider the measure

$$
d \dot{\lambda}(z)=\dot{W}(z)^{-\epsilon p} d \lambda(z)
$$

on $\underset{\sim}{\dot{Z}}$. Let $L_{n}^{2}(\underset{\sim}{\dot{Z}})$ denote the $L^{2}$-space on $\underset{\sim}{Z}$ for the measure

$$
\dot{C}_{n} \dot{W}(z)^{n} d \dot{\lambda}(z)=\dot{C}_{n} \dot{W}(z)^{n-\epsilon p} d \lambda(z)
$$

and define the weighted Bergman space

$$
H_{n}^{2}(\underset{\sim}{\dot{Z}}):=L_{n}^{2}(\underset{\sim}{\dot{Z}}) \cap \operatorname{Hol}(\underset{\sim}{Z}) .
$$

Let $\underset{\sim}{\dot{K}_{n}}(z, w)$ be the corresponding reproducing kernel function.

Proposition 7.2. For $n \rightarrow+\infty$ we have

$$
\frac{\dot{K}_{n}(z, z)}{\dot{K}_{n}(z, z)} \asymp 1, \quad \frac{\dot{K}_{n}(z, w)}{\dot{K}_{n}(z, z)^{1 / 2} \dot{K}_{n}(w, w)^{1 / 2}} \asymp \underset{\sim}{\dot{K}_{n}(z, z)^{1 / 2} \dot{\sim}_{n}(w, w)^{1 / 2}},
$$

uniformly for $\|z\|,\|w\| \leq 1 / 4$.

Proof. Consider the Hartogs domains,

$$
\begin{gathered}
\dot{\mathcal{D}}:=\left\{(z, t) \in \dot{Z} \times \mathbf{C}:|t|^{2}<\dot{K}(z, z)^{-1}\right\}, \\
\dot{\mathcal{D}}=\left\{(z, t) \in \underset{\sim}{Z} \times \mathbf{C}:|t|^{2}<\dot{W}(z)\right\} .
\end{gathered}
$$

As a rule we use undertilde notation for objects associated with the local model. Putting

$$
\alpha:=\epsilon^{2}\left(1-\frac{1-\epsilon}{2} p\right)
$$


it follows that

$$
n=k+1+\epsilon p-\alpha, k \in \mathbf{N},
$$

ranges precisely over the integers in the parameter range. We have

$$
\begin{aligned}
& \int_{\dot{\mathcal{D}}} d \lambda(z, t) \dot{K}(z, z)^{\alpha} t^{k} \phi(z) \overline{t^{l} \psi(z)}=\delta_{k l} \frac{\pi}{k+1} \int_{\dot{Z}} d \lambda(z) \dot{K}(z, z)^{\alpha} \phi(z) \overline{\psi(z)} \dot{K}(z, z)^{-k-1} \\
& =\delta_{k l} \frac{\pi}{k+1} \int_{\dot{Z}} d \lambda(z) \phi(z) \overline{\psi(z)} \dot{K}(z, z)^{\alpha-k-1}=\delta_{k l} \frac{\pi}{k+1} \int_{\dot{Z}} d \dot{\lambda}(z) \phi(z) \overline{\psi(z)} \dot{K}(z, z)^{-n}
\end{aligned}
$$

and

$$
\begin{aligned}
& \int_{\underset{\mathcal{D}}{\dot{\mathcal{D}}}} d \lambda(z, t) \dot{W}(z)^{-\alpha} t^{k} \phi(z) \overline{t^{l} \psi(z)}=\delta_{k l} \frac{\pi}{k+1} \int_{\underset{\sim}{Z}} d \lambda(z) \dot{W}(z)^{-\alpha} \phi(z) \overline{\psi(z)} \dot{W}(z)^{k+1} \\
& =\delta_{k l} \frac{\pi}{k+1} \int_{\underset{\sim}{Z}} d \lambda(z) \phi(z) \overline{\psi(z)} \dot{W}(z)^{k+1-\alpha}=\delta_{k l} \frac{\pi}{k+1} \int_{\underset{\sim}{Z}} d \dot{\lambda}(z) \phi(z) \overline{\psi(z)} \dot{W}(z)^{n} .
\end{aligned}
$$

Therefore the corresponding Bergman spaces

$$
\begin{aligned}
A^{2}(\dot{\mathcal{D}}) & :=\operatorname{Hol}(\dot{\mathcal{D}}) \cap L^{2}\left(\dot{\mathcal{D}}, d \lambda(z, t) \dot{K}(z, z)^{\alpha}\right), \\
A^{2}(\underset{\mathcal{D}}{\dot{\mathcal{D}}}) & :=\operatorname{Hol}(\underset{\sim}{\dot{\mathcal{D}}}) \cap L^{2}\left(\underset{\mathcal{D}}{\dot{\mathcal{L}}}, d \lambda(z, t) \dot{W}(z)^{-\alpha}\right)
\end{aligned}
$$

have a decomposition

$$
A^{2}(\dot{\mathcal{D}})=\bigoplus_{n} A^{2}\left(\dot{Z}, \dot{K}(z, z)^{-n} d \dot{\lambda}(z)\right), \quad A^{2}(\underset{\sim}{\dot{\mathcal{D}}})=\bigoplus_{n} A^{2}\left(\underset{\sim}{Z}, \dot{W}(z)^{n} d \dot{\lambda}(z)\right)
$$

into a direct sum of weighted Bergman spaces which have reproducing kernels $\dot{C}_{n} \dot{K}_{n}(z, w)$ and $\dot{C}_{n}{\underset{\sim}{K}}_{n}(z, w)$, respectively. It follows [FR, [Li] that the corresponding kernel functions, denoted by $\dot{\mathcal{K}}$ and $\underset{\sim}{\mathcal{K}}$, respectively, have decompositions

$$
\begin{aligned}
& \dot{\mathcal{K}}((z, t),(w, s))=\sum_{k \in \mathbf{N}} \frac{k+1}{\pi}(t \bar{s})^{k} C_{n} \dot{K}_{n}(z, w), \\
& \dot{\mathcal{K}}((z, t),(w, s))=\sum_{k \in \mathbf{N}} \frac{k+1}{\pi}(t \bar{s})^{k} C_{n} \dot{\sim}_{n}(z, w) .
\end{aligned}
$$

The unit disc bundle $\mathbf{B}(\dot{Z})$ of the dual canonical line bundle over $\dot{Z}$ agrees with $\dot{\mathcal{D}}$ (in the compact case, this holds up to the fiber over $\hat{Z} \backslash Z$ which has Lebesgue measure zero). As in [EZ] it follows that the Bergman spaces of $\mathbf{B}(\dot{Z})$ and $\dot{\mathcal{D}}$ are the same by a variant of the Riemann removable singularity theorem (cf. the footnote on p. 905 in $[\mathrm{EZ}]$ ). Hence $\dot{\mathcal{K}}$ agrees with (the restriction of) the Bergman kernel of $\mathbf{B}(\dot{Z})$. On the other hand Lemma 5 in E1] (which remains valid - with the same proof - also for domains in non-singular complex manifolds) shows that the Bergman kernels of $\underset{\sim}{\dot{\mathcal{D}}}$ and $\mathbf{B}(\dot{Z})$ differ by a smooth function on the compact subset $\|z\|,\|w\| \leq 1 / 3$. It follows that the difference $\dot{\mathcal{K}}-\dot{\mathcal{K}}$ is smooth on the closed set

$$
\left\{((z, t),(w, s)):\|z\| \leq 1 / 3,|t|^{2} \leq \dot{W}(z),\|w\| \leq 1 / 3,|s|^{2} \leq \dot{W}(w)\right\} .
$$

By the familiar Cauchy estimates, a holomorphic function $f(z)=\sum_{n=0}^{\infty} f_{n} z^{n}$ on the unit disc has Taylor coefficients $f_{n}=O\left(n^{-\infty}\right)$ if and only if it is smooth on the closed unit disc. Using (7.3), (7.4) and accommodating for the fact that $(t \bar{s})$ lies 
not in the unit disc but in the disc $|t \bar{s}|^{2}<\dot{W}(z) \dot{W}(w)$ whose radius varies with $z, w$, it follows that for $n \rightarrow+\infty$

$$
\frac{\dot{\sim}_{n}(z, w)-\dot{K}_{n}(z, w)}{\dot{W}(z)^{-n / 2} \dot{W}(w)^{-n / 2}}=O\left(n^{-\infty}\right)
$$

uniformly for $\|z\|,\|w\| \leq 1 / 4$. Since $\dot{W}(z)=\dot{K}(z, z)^{-1}$ for such $z$, taking $z=w$ gives the first formula in (7.1), and feeding it back into (7.5) also gives the second formula in (7.1).

Remark. With a little more labor, it can be shown that even

$$
\frac{\dot{\mathscr{K}}_{n}(z, w)}{\dot{K}_{n}(z, w)} \asymp 1
$$

uniformly for $\|z\|,\|w\| \leq 1 / 4$; cf. formula (24) and the remarks after it on p. 224 in E1. For $z \neq w$, this is an improvement by the exponential factor of $\left[\dot{K}(z, w) \dot{K}(z, z)^{-1 / 2} \dot{K}(w, w)^{-1 / 2}\right]^{n}$ against (7.1). However, we will not really need this refinement in the sequel.

The local model also has real forms $\underset{\sim}{\dot{Z}}$. In the real case the conjugation $z \mapsto z^{\sharp}$ of $Z$ satisfies

$$
\begin{aligned}
\left(z^{\sharp} \mid w^{\sharp}\right) & =(w \mid z), \\
\dot{K}\left(z^{\sharp}, w^{\sharp}\right) & =\dot{K}(w, z) .
\end{aligned}
$$

Therefore one may choose the defining function $\dot{W}$ for the local model $\underset{\sim}{\dot{Z}}$ such that

$$
\dot{W}\left(z^{\sharp}\right)=\dot{W}(z) \text {. }
$$

Making such a choice, we obtain the real form ${\underset{\sim}{Z}}_{\mathbf{R}}=Z_{\mathbf{R}} \cap \underset{\sim}{\dot{Z}}$. In the complex case we put $\dot{\sim}_{\mathbf{C}}:=\underset{\sim}{\dot{Z}}$, considered as a real domain. Endow $\dot{\sim}_{\mathbf{K}}$ with the measure

$$
d \dot{\sim}_{\mathbf{K}}(x)=\dot{W}(x)^{-\epsilon \kappa p / 2} d \lambda_{\mathbf{K}}(x) .
$$

Passing to the complexification $Z^{\mathbf{K}}$ we have as in the symmetric case

$$
\begin{aligned}
& {\underset{\sim}{Z}}^{\mathbf{R}}=\underset{\sim}{Z}, \quad{\underset{\sim}{Z}}^{\mathbf{C}}=\underset{\sim}{Z} \times \underset{\sim}{\bar{Z}}=\left\{\left(z_{1}, \bar{z}_{2}\right): z_{1}, z_{2} \in \underset{\sim}{Z}\right\}, \\
& \dot{\sim}^{\mathbf{R}}=\underset{\sim}{\dot{Z}}, \quad \dot{\sim}^{\mathbf{C}}=\underset{\sim}{\dot{Z}} \times \underset{\sim}{\dot{Z}}=\left\{\left(z_{1}, \bar{z}_{2}\right): z_{1}, z_{2} \in \underset{\sim}{\dot{Z}}\right\}, \\
& \dot{W}^{\mathbf{R}}(z)=\dot{W}(z), \quad \dot{W}^{\mathbf{C}}(z, w)=\dot{W}(z) \dot{W}(w), \\
& d \dot{\sim}^{\mathbf{R}}(z)=d \dot{\sim} \underset{\sim}{\dot{\lambda}}(z), \quad d \dot{\sim}^{\mathbf{C}}(z, \bar{w})=d \underset{\sim}{\dot{\lambda}}(z) d \underset{\sim}{\dot{\lambda}}(w), \\
& H_{n}^{2}\left(\dot{\sim}^{\mathbf{R}}\right)=H_{n}^{2}(\underset{\sim}{\dot{Z}}), \quad H_{n}^{2}\left({\underset{\sim}{\dot{Z}}}^{\mathbf{C}}\right)=H_{n}^{2}(\underset{\sim}{\dot{Z}} \times \underset{\sim}{\dot{Z}})=H_{n}^{2}(\underset{\sim}{\dot{Z}}) \otimes \overline{H_{n}^{2}(\dot{\dot{Z}})}, \\
& {\underset{\sim}{K}}_{n}^{\mathbf{R}}(z, w)=\dot{\sim}_{n}(z, w), \quad \dot{\sim}_{n}^{\mathbf{C}}\left(z_{1}, \bar{z}_{2}, w_{1}, \bar{w}_{2}\right)=\underset{\sim}{\dot{K}_{n}}\left(z_{1}, w_{1}\right) \underset{\sim}{\dot{K}_{n}}\left(w_{2}, z_{2}\right) .
\end{aligned}
$$

Note that ${\underset{\sim}{Z}}^{\mathbf{C}}$ is not the unit ball of $Z^{\mathbf{C}}$. 
We define the Berezin symbol $\dot{\sigma}_{\mathbf{K}}^{\nu}$, Toeplitz map $\dot{\sim}_{\mathbf{K}}^{\nu}$ and Berezin transform $\dot{\sim}_{\mathbf{K}}^{\nu}$ as above using the measure

$$
\dot{C}_{\mathbf{K}}^{\nu} d \dot{\sim}_{\mathbf{K}}(x)=\dot{C}_{\mathbf{K}}^{\nu} \dot{W}(x)^{-\epsilon p \kappa / 2} d \lambda_{\mathbf{K}}(x)
$$

on ${\underset{\sim}{\mathbf{Z}}}_{\mathbf{K}}$. Thus

$$
\begin{aligned}
& \left(\dot{\sim}_{\mathbf{K}}^{\nu} f\right)(x)=\dot{C}_{\mathbf{K}}^{\nu} \int_{\sim_{\mathbf{K}}} d \dot{\sim}_{\mathbf{K}}(y) \frac{\dot{\sim}_{n}^{\mathbf{K}}(x, y)}{\dot{\sim}_{n}(x, x)^{\kappa / 2} \underset{\sim}{\dot{K}} \dot{\sim}_{n}(y, y)^{\kappa / 2}} f(y) \\
= & \dot{C}_{\mathbf{K}}^{\nu} \int_{{\underset{\sim}{\mathbf{K}}}_{\mathbf{K}}} d \lambda_{\mathbf{K}}(y) \dot{W}(x)^{-\epsilon p \kappa / 2} \frac{\dot{\sim}_{n}^{\mathbf{K}}(x, y)}{{\underset{\sim}{K}}_{n}(x, x)^{\kappa / 2}{\underset{\sim}{K}}_{n}(y, y)^{\kappa / 2}} f(y) .
\end{aligned}
$$

Proposition 7.3. The Berezin transform for $\dot{Z}$ and its local model $\underset{Z}{\dot{Z}}$ have the same asymptotic expansion near 0 . More precisely, if $f \in \mathcal{C}^{\infty}\left(Z_{\mathbf{K}}\right)$ has support $\|\operatorname{supp} f\| \leq 1 / 4$, then

$$
\left(\dot{\beta}_{\mathbf{K}}^{\nu} f\right)(x) \asymp\left({\underset{\sim}{\mathbf{K}}}_{\mathbf{K}}^{\nu} f\right)(x)
$$

uniformly for all $x \in Z_{\mathbf{K}}$ satisfying $\|x\| \leq 1 / 4$.

Proof. By (7.1) we have for $x, y \in Z_{\mathbf{K}}$ with $\|x\|,\|y\| \leq 1 / 4$,

$$
d \dot{\lambda}_{\mathbf{K}}(y)=d \dot{\lambda}_{\mathbf{K}}(y)
$$

and

$$
\frac{\dot{\sim}_{n}^{\mathbf{K}}(x, y)}{{\underset{\sim}{K}}_{n}(x, x)^{\kappa / 2}{\underset{\sim}{K}}_{n}(y, y)^{\kappa / 2}}=\frac{\dot{K}_{n}^{\mathbf{K}}(x, y)}{\dot{K}(x, x)^{n \kappa / 2} \dot{K}(y, y)^{n \kappa / 2}}+h_{n}(x, y),
$$

where

$$
\left\|h_{n}\right\|:=\sup _{\|x\| \leq 1 / 4,\|y\| \leq 1 / 4}\left|h_{n}(x, y)\right| \asymp 0 .
$$

Therefore the assumption $\|\operatorname{supp} f\| \leq 1 / 4$ implies

$$
\begin{aligned}
\left|\dot{\beta}_{\mathbf{K}}^{\nu} f(x)-\dot{\beta}_{\mathbf{K}}^{\nu} f(x)\right| & \\
= & \dot{C}_{\mathbf{K}}^{\nu} \mid \int d \dot{\sim}_{\mathbf{K}}(y) \frac{{\underset{\sim}{n}}_{n}(x, x)^{\kappa / 2} \dot{\sim}_{n}(y, y)^{\kappa / 2}}{\dot{K}_{n}} f(y) \\
& -\int d \dot{\lambda}_{\mathbf{K}}(y) \frac{\dot{K}_{n}^{\mathbf{K}}(x, y)}{\dot{K}(x, x)^{n \kappa / 2} \dot{K}(y, y)^{n \kappa / 2}} f(y) \mid \\
= & \dot{C}_{\mathbf{K}}^{\nu}\left|\int d \dot{\lambda}_{\mathbf{K}}(y) h_{n}(x, y) f(y)\right| \\
& \leq\left\|h_{n}\right\| \dot{C}_{\mathbf{K}}^{\nu} \int d \dot{\lambda}_{\mathbf{K}}(y) f(y) .
\end{aligned}
$$

Since

$$
\dot{C}_{\mathbf{K}}^{\nu} \approx \text { const. } n^{\kappa d / 2}
$$

grows like a fixed power of $n$ we have $\dot{C}_{\mathbf{K}}^{\nu}\left\|h_{n}\right\| \asymp 0$, and (7.6) follows. 
As a special case we have

Proposition 7.4. The Berezin operator transform for $\dot{Z}$ and its local model $\underset{Z}{\dot{Z}}$ have the same asymptotic expansion near 0 . More precisely, if $f \in \mathcal{C}^{\infty}(Z)$ satisfies $\|\operatorname{supp} f\| \leq 1 / 4$, then

$$
\left(\dot{B}_{n} f\right)(z) \asymp\left(\dot{\sim}_{n} f\right)(z)
$$

uniformly for all $z \in Z$ with $\|z\| \leq 1 / 4$.

Define the holomorphic function ${\underset{\sim}{\sim}}_{\mathrm{K}}^{\nu}$ and the complexified Toeplitz operators $\dot{\sim}_{n}^{\mathrm{K}} F$ as in the symmetric case. As in (6.4) this yields the "balanced" star-operation

$$
\begin{aligned}
& \dot{\sigma}_{\mathbf{K}}^{\nu}\left(\left(\dot{\sim}_{n}^{\mathbf{K}} F\right) \dot{\sim}_{\mathbf{K}}^{\nu}\right)(x)=\dot{C}_{n}^{\kappa} \int_{Z^{\mathbf{K}}} d \dot{\sim}^{\mathbf{K}}(w) \dot{W}^{\mathbf{K}}(w)^{n} \frac{\dot{\dot{K}}_{n}^{\mathbf{K}}(x, w) \dot{\dot{I}}_{\mathbf{K}}^{\nu}(w)}{\dot{\sim}_{\mathbf{K}}^{\nu}(x)} F(w) \\
& =\dot{C}_{n}^{\kappa} \int_{\underline{Z}^{\mathbf{K}}} d \lambda^{\mathbf{K}}(w) \dot{W}^{\mathbf{K}}(w)^{n-\epsilon p} \frac{\dot{\underline{K}}_{n}^{\mathbf{K}}(x, w) \dot{\sim}_{\mathbf{K}}^{\nu}(w)}{\dot{\dot{I}}_{\mathbf{K}}^{\nu}(x)} F(w) .
\end{aligned}
$$

Proposition 7.5. The balanced star-operation for $\dot{Z}_{\mathbf{K}}$ and its local model ${\underset{\sim}{\mathbf{K}}}_{\mathbf{K}}$ have the same asymptotic expansion near 0 . More precisely, if $F \in \mathcal{C}^{\infty}\left(Z^{\mathbf{K}}\right)$ has support $\|\operatorname{supp} F\| \leq 1 / 4$, then

$$
\dot{\sigma}_{\mathbf{K}}^{\nu}\left(\left(\dot{T}_{n}^{\mathbf{K}} F\right) \dot{I}_{\mathbf{K}}^{\nu}\right)(x) \asymp \dot{\sigma}_{\mathbf{K}}^{\nu}\left(\left(\dot{\sim}_{n}^{\mathbf{K}} F\right) \dot{\sim}_{\mathbf{K}}^{\nu}\right)(x)
$$

uniformly for all $x \in Z_{\mathbf{K}}$ satisfying $\|x\| \leq 1 / 4$.

Proof. By (7.1) we have for all $x \in Z_{\mathbf{K}}, w \in Z^{\mathbf{K}}$ with $\|x\|,\|w\| \leq 1 / 4$,

$$
\begin{gathered}
d \dot{\sim}^{\mathbf{K}}(w)=d \dot{\lambda}^{\mathbf{K}}(w), \\
\dot{W}^{\mathbf{K}}(w)^{n}=\dot{K}^{\mathbf{K}}(w, w)^{-n}
\end{gathered}
$$

and

$$
\begin{aligned}
& \frac{\dot{\sim}_{n}^{\mathbf{K}}(x, w) \dot{\dot{I}}_{\mathbf{K}}^{\nu}(w)}{\dot{\sim}_{\mathbf{K}}^{\nu}(x) \dot{K}_{n}(w, w)}-\frac{\dot{K}_{n}^{\mathbf{K}}(x, w) \dot{I}_{\mathbf{K}}^{\nu}(w)}{\dot{I}_{\mathbf{K}}^{\nu}(x) \dot{K}_{n}(w, w)} \\
& =\frac{\dot{\sim}_{n}(x, w)}{{\underset{\sim}{K}}_{n}(x, x)^{1 / 2} \underset{\sim}{\dot{\sim}_{n}}(w, w)^{1 / 2}} \frac{\dot{\boldsymbol{K}}_{n}(w, w)}{\dot{K}_{n}(w, w)}\left[\frac{\dot{\sim}_{n}\left(w, w^{\#}\right)}{{\underset{\sim}{K}}_{n}(w, w)^{1 / 2}{\underset{\sim}{K}}_{n}\left(w^{\#}, w^{\#}\right)^{1 / 2}}\right]^{1 / 2} \\
& -\frac{\dot{K}_{n}(x, w)}{\dot{K}_{n}(x, x)^{1 / 2} \dot{K}_{n}(w, w)^{1 / 2}}\left[\frac{\dot{K}_{n}\left(w, w^{\#}\right)}{\dot{K}_{n}(w, w)^{1 / 2} \dot{K}_{n}\left(w^{\#}, w^{\#}\right)^{1 / 2}}\right]^{1 / 2} \\
& =h_{n}(x, w) \text {, }
\end{aligned}
$$

where

$$
\left\|h_{n}\right\|:=\sup _{\|x\| \leq 1 / 4,\|w\| \leq 1 / 4}\left|h_{n}(x, w)\right| \asymp 0 .
$$

Therefore the assumption $\|\operatorname{supp} F\| \leq 1 / 4$ implies

$$
\begin{aligned}
& \left|\dot{\sigma}_{\mathbf{K}}^{\nu}\left(\left(\dot{\sim}_{n}^{\mathbf{K}} F\right) \dot{\sim}_{\mathbf{K}}^{\nu}\right)(x)-\dot{\sigma}_{\mathbf{K}}^{\nu}\left(\left(\dot{T}_{n}^{\mathbf{K}} F\right) \dot{I}_{\mathbf{K}}^{\nu}\right)(x)\right| \\
& \quad=\dot{C}_{n}^{\kappa} \mid \int d \dot{\sim}^{\mathbf{K}}(w) \dot{W}^{\mathbf{K}}(w) \frac{\dot{K}_{n}^{\mathbf{K}}(x, w) \dot{\sim}_{\mathbf{K}}^{\nu}(w)}{\dot{\sim}_{\mathbf{K}}^{\nu}(x)} F(w)
\end{aligned}
$$




$$
\begin{aligned}
& -\int d \dot{\lambda}^{\mathbf{K}}(w) \dot{K}^{\mathbf{K}}(w, w)^{-n} \frac{\dot{K}_{n}^{\mathbf{K}}(x, w) \dot{I}_{\mathbf{K}}^{\nu}(w)}{\dot{I}_{\mathbf{K}}^{\nu}(x)} F(w) \mid \\
= & \dot{C}_{n}^{\kappa}\left|\int d \dot{\lambda}^{\mathbf{K}}(w) h_{n}(x, w) F(w)\right| \leq \dot{C}_{n}^{\kappa}\left\|h_{n}\right\| \int d \dot{\lambda}^{\mathbf{K}}(w)|F(w)| .
\end{aligned}
$$

Since $\dot{C}_{n}^{\kappa} \approx$ const. $n^{\kappa d / 2}$ grows like a fixed power of $n, \dot{C}_{n}^{\kappa}\left\|h_{n}\right\|=O\left(n^{-\infty}\right)$, and the assertion follows.

As a special case we obtain

Proposition 7.6. The balanced star-product (6.15) for $\dot{Z}$ and its local model $\dot{Z}$ have the same asymptotic expansion near 0 . More precisely, if $f_{1}, f_{2} \in \mathcal{C}^{\infty}(Z)$ satisfy $\left\|\operatorname{supp} f_{i}\right\| \leq 1 / 4$, then

$$
\dot{S}_{n}\left(\left(\dot{T}_{n} f_{1}\right)\left(\dot{T}_{n} f_{2}\right)\right)(z) \asymp \dot{\sim}_{n}\left(\left(\dot{\sim}_{n} f_{1}\right)\left(\dot{T}_{n} f_{2}\right)\right)(z)
$$

uniformly for all $z \in Z$ with $\|z\| \leq 1 / 4$.

\section{THE FACTORIZATION PROPERTY}

Using the local model introduced above allows us to prove the crucial (anti-) holomorphic factorization properties in all cases including the compact type which has no global holomorphic functions. The Berezin and Toeplitz star-reducts behave in a dual manner; we will first treat the Toeplitz case.

Every $K_{\mathbf{K}}$ invariant polynomial $p$ on $Z_{\mathbf{K}}$ has a unique holomorphic extension to $Z^{\mathbf{K}}$ whose associated constant coefficient differential operator $p\left(\partial^{\mathbf{K}}\right)$ contains only holomorphic derivatives. Since $p\left(\partial^{\mathbf{K}}\right)$ is invariant under $K_{\mathbf{K}} \subset K^{\mathbf{K}}$, acting on $Z_{\mathbf{K}} \subset Z^{\mathbf{K}}$, we obtain a differential operator $p\left(\dot{\partial}^{\mathbf{K}}\right)$ from $\dot{Z}^{\mathbf{K}}$ to $\dot{Z}_{\mathbf{K}}$, which is invariant under $\dot{G}_{\mathbf{K}} \subset \dot{G}^{\mathbf{K}}$, acting on $\dot{Z}_{\mathbf{K}} \subset \dot{Z}^{\mathbf{K}}$, by putting

$$
\left(p\left(\dot{\partial}^{\mathbf{K}}\right) F\right)(x)=p\left(\partial^{\mathbf{K}}\right)\left(F \circ \dot{\mathfrak{t}}_{x}\right)(0)
$$

for all $x \in \dot{Z}_{\mathbf{K}}$. Here $\dot{\mathfrak{t}}_{x} \in G_{\mathbf{K}}$ are the transvections (2.5).

The next theorem shows that the Toeplitz star-reduct is essentially the holomorphic extension of the inverse Berezin transform.

Theorem 8.1. Consider the $K_{\mathbf{K}}$-invariant real polynomials $q_{j}$ on $Z_{\mathbf{K}}$ in the asymptotic expansion (5.9) of the inverse Berezin transform. Then the Toeplitz star-reduct has the asymptotic expansion as $\nu \rightarrow \infty$ :

$$
\left(\dot{*}_{\nu}^{\mathbf{K}} F\right)(x) \asymp \sum_{j=0}^{\infty} \frac{\left(q_{j}\left(\dot{\partial}^{\mathbf{K}}\right) F\right)(x)}{\nu^{j}}
$$

for all $x \in \dot{Z}_{\mathbf{K}}$. The differential operators $\mathcal{L}_{j}=q_{j}\left(\dot{\partial}^{\mathbf{K}}\right)$ involve only holomorphic derivatives and have the anti-holomorphic factorization property

$$
\mathcal{L}_{j}(\bar{H} F)(x)=\overline{H(x)} \mathcal{L}_{j} F(x)
$$

for all functions $H \in \mathcal{C}^{\infty}\left(Z^{\mathbf{K}}\right)$ holomorphic in a neighborhood of $x \in Z_{\mathbf{K}}$. The 0 -order term $\mathcal{L}_{0}=i d$ is the identity map.

Proof. The crucial step is to prove the property (8.1) which reflects the well-known behavior of Toeplitz operators with respect to bounded holomorphic functions. We pass to the local model, using undertilde notation for the associated objects. Suppose that $F \in \mathcal{C}^{\infty}\left(\underline{\sim}^{\mathbf{K}}\right)$ has support $\operatorname{supp} F$ of norm $\leq 1 / 4$ and $x \in Z_{\mathbf{K}}$ satisfies $\|x\| \leq 1 / 4$. Proposition 7.3 and Proposition 7.5 imply that the Toeplitz star-reduct 
$\underset{\sim}{\dot{*}_{\nu}^{\mathbf{K}}}$ associated with the local model ${\underset{\sim}{\dot{Z}}}_{\mathbf{K}}$ has the same asymptotic expansion as $\nu \rightarrow \infty$ :

$$
\left(\stackrel{*}{*}_{\nu}^{\mathbf{K}} F\right)(x) \asymp \sum_{j=0}^{\infty} \frac{\left(\mathcal{L}_{j} F\right)(x)}{\nu^{j}}
$$

as in (6.11). Let $H$ be a bounded holomorphic function on $\underset{\sim}{Z}$ and $\Phi, \Psi \in H_{n}^{2}\left(\dot{Z}^{\mathbf{K}}\right)$. Let $\rho$ denote the restriction map onto $\dot{Z}_{\mathbf{K}}$. The identity

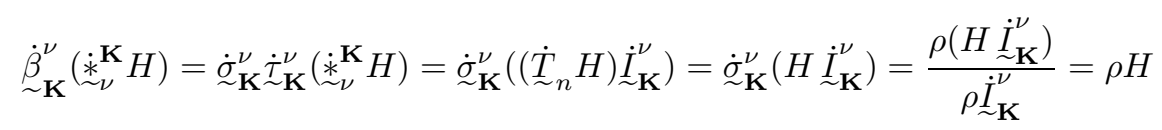

implies for all $j$

$$
\left(\mathcal{L}_{j} H\right)(0)=\left(q_{j}\left(\partial_{\mathbf{K}}\right) \rho H\right)(0)=\left(q_{j}\left(\partial^{\mathbf{K}}\right) H\right)(0) .
$$

Since

$$
\begin{gathered}
\left(\Phi \mid \dot{\tau}_{\mathbf{K}}^{\nu}(\overline{\rho H} F)\right)=\left(\dot{\sim}_{\mathbf{K}}^{\nu} \Phi \mid \overline{\rho H} F\right)=\left((\rho H) \dot{\tilde{\sigma}}_{\mathbf{K}}^{\nu} \Phi \mid F\right)=\left(\dot{\sigma}_{\mathbf{K}}^{\nu}(H \Phi) \mid F\right) \\
=\left(H \Phi \mid \dot{\tau}_{\mathbf{K}}^{\nu} F\right)=\left(\Phi \mid \bar{H} \dot{\sim}_{\mathbf{K}}^{\nu} F\right)=\left(\Phi \mid\left(\dot{\sim}_{n}^{\mathbf{K}} \bar{H}\right) \dot{\sim}_{\mathbf{K}}^{\nu} F\right),
\end{gathered}
$$

we have

$$
\dot{\sim}_{\mathbf{K}}^{\nu}(\overline{\rho H} F)=\left(\dot{\sim}_{n}^{\mathbf{K}} \bar{H}\right)\left(\dot{\tau}_{\mathbf{K}}^{\nu} F\right)
$$

Since

we have

$$
\begin{gathered}
\left(\Phi \mid\left(\dot{\sim}_{n}^{\mathbf{K}} \bar{H}\right)\left(\dot{\sim}_{n}^{\mathbf{K}} F\right) \Psi\right)=\left(\Phi \mid \bar{H}\left(\dot{\sim}_{n}^{\mathbf{K}} F\right) \Psi\right)=\left(H \Phi \mid\left(\dot{\sim}_{n}^{\mathbf{K}} F\right) \Psi\right) \\
=(H \Phi \mid F \Psi)=(\Phi \mid \bar{H} F \Psi)=\left(\Phi \mid \dot{\sim}_{n}^{\mathbf{K}}(\bar{H} F) \Psi\right),
\end{gathered}
$$

$$
\dot{\sim}_{n}^{\mathbf{K}}(\bar{H} F)=\left({\underset{\sim}{\sim}}_{n}^{\mathbf{K}} \bar{H}\right)\left(\dot{\sim}_{n}^{\mathbf{K}} F\right) .
$$

Combining (8.2) and (8.3) it follows that

$$
\begin{gathered}
\dot{\sim}_{\mathbf{K}}^{\nu}\left(\dot{*}_{\nu}^{\mathbf{K}}(\bar{H} F)\right) \asymp{\underset{\sim}{\dot{T}}}_{n}^{\mathbf{K}}(\bar{H} F) \dot{\sim}_{\mathbf{K}}^{\nu}=\left(\dot{\sim}_{n}^{\mathbf{K}} \bar{H}\right)\left({\underset{\sim}{T}}_{n}^{\mathbf{K}} F\right) \dot{\sim}_{\mathbf{K}}^{\nu} \\
\asymp\left({\underset{\sim}{T}}_{n}^{\mathbf{K}} \bar{H}\right) \dot{\sim}_{\mathbf{K}}^{\nu}\left(\dot{*}_{\nu}^{\mathbf{K}} F\right) \asymp \dot{\sim}_{\mathbf{K}}^{\nu}\left(\overline{\rho H}\left(\stackrel{\sim}{*}_{\nu}^{\mathbf{K}} F\right)\right) .
\end{gathered}
$$

Since $\dot{\tau}_{\mathbf{K}}^{\nu}$ is injective in the bounded setting, we obtain

$$
\stackrel{\dot{*}}{\sim}_{\nu}^{\mathbf{K}}(\bar{H} F) \asymp \overline{\rho H}\left(\dot{*}_{\nu}^{\mathbf{K}} F\right) \text {. }
$$

This implies for each $j$

$$
\mathcal{L}_{j}(\bar{H} F)(0)=\overline{H(0)}\left(\mathcal{L}_{j} F\right)(0),
$$

proving (8.1) for $x=0$. Also

$$
\mathcal{L}_{j}(\Phi \bar{\Psi})(0)=\overline{\Psi(0)}\left(\mathcal{L}_{j} \Phi\right)(0)=\overline{\Psi(0)}\left(q_{j}\left(\partial^{\mathbf{K}}\right) \Phi\right)(0)=q_{j}\left(\partial^{\mathbf{K}}\right)(\Phi \bar{\Psi})(0),
$$

showing that

$$
\left(\mathcal{L}_{j} F\right)(0)=\left(q_{j}\left(\partial^{\mathbf{K}}\right) F\right)(0)
$$

for all $j$. Since $\left(q_{j}\left(\partial^{\mathbf{K}}\right) F\right)(0)$ depends only on the jet of $F$ at 0 , (8.4) remains in force even if $H$ is holomorphic only in some neighborhood of 0 (not necessarily on all of $\underset{\sim}{Z}$ ). Now (8.1) follows from $\dot{G}_{\mathbf{K}}$ invariance, using the fact that the transvections $\dot{\mathfrak{t}}_{x}$ are holomorphic on $\dot{Z}^{\mathrm{K}}$.

The next theorem shows that the Berezin star-reduct is essentially the antiholomorphic extension of the Berezin transform. 
Theorem 8.2. Consider the $K_{\mathbf{K}}$-invariant real polynomials $p_{j}$ on $Z_{\mathbf{K}}$ in the asymptotic expansion (5.6) of the Berezin transform. Then the Berezin star-reduct has the asymptotic expansion as $\nu \rightarrow \infty$ :

$$
\left(\overline{\dot{*}}_{\nu}^{\mathbf{K}} F\right)(x)=\sum_{j=0}^{\infty} \frac{\left(\overline{p_{j}\left(\dot{\partial}^{\mathbf{K}}\right)} F\right)(x)}{\nu^{j}}
$$

for all $x \in \dot{Z}_{\mathbf{K}}$. The differential operators $\mathcal{N}_{j}:=\overline{p_{j}(\dot{\partial} \mathbf{K})}$ involve only anti-holomorphic derivatives and have the holomorphic factorization property

$$
\mathcal{N}_{j}(H F)(x)=H(x)\left(\mathcal{N}_{j} F\right)(x)
$$

for all functions $H \in \mathcal{C}^{\infty}\left(Z^{\mathbf{K}}\right)$ holomorphic in a neighborhood of $x \in Z_{\mathbf{K}}$. The 0 -order term $\mathcal{N}_{0}=i d$ is the identity map.

Proof. Again, the crucial property (8.5) reflects the well-known behavior of the Berezin operator symbol with respect to bounded holomorphic functions. We pass to the local model, using undertilde notation for the associated objects. Suppose $F \in \mathcal{C}^{\infty}\left({\underset{\sim}{Z}}^{\mathbf{K}}\right)$ has support $\operatorname{supp} F$ of norm $\leq 1 / 4$ and $x \in Z_{\mathbf{K}}$ satisfies $\|x\| \leq$ 1/4. Proposition 7.3 and Proposition 7.5 imply that the Berezin star-reduct $\underset{\sim}{\stackrel{\dot{*}}{\mathbf{K}}}$ associated to the local model $\underset{\sim}{\dot{Z}}$ has the same asymptotic expansion as $\nu \rightarrow \infty$ :

$$
\left(\stackrel{(\stackrel{*}{*}}{\sim}_{\nu}^{\mathbf{K}} F\right)(x) \asymp \sum_{j=0}^{\infty} \frac{\left(\mathcal{N}_{j} F\right)(x)}{\nu^{j}}
$$

as in (6.10). Let $H$ be a bounded holomorphic function on $\underset{\sim}{Z}$ and $\Phi, \Psi \in H_{n}^{2}\left(\dot{\sim}^{\mathbf{K}}\right)$. The identity

$$
{\underset{\sim}{\dot{K}}}_{n}^{\mathbf{K}}(z, z)\left({\underset{\sim}{\dot{B}}}_{n}^{\mathbf{K}} \bar{H}\right)(z)=\left(\left({\underset{\sim}{\dot{T}}}_{n}^{\mathbf{K}} \bar{H}\right) \dot{\sim}_{z}^{n} \mid \dot{\sim}_{z}^{n}\right)=\left({\underset{\sim}{\dot{K}}}_{z}^{n} \mid H{\underset{\sim}{\dot{K}}}_{z}^{n}\right)=\overline{H(z)} \dot{\sim}_{n}^{\mathbf{K}}(z, z)
$$

implies

$$
\dot{\sim}_{n}^{\mathrm{K}} \bar{H}=\bar{H}
$$

Therefore

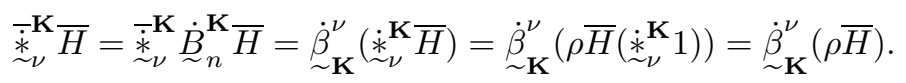

As a consequence

$$
\left(\mathcal{N}_{j} \bar{H}\right)(0)=p_{j}\left(\partial_{\mathbf{K}}\right)(\rho \bar{H})(0) .
$$

Let $M_{H}$ denote the multiplication operator on $H_{n}^{2}\left(\dot{\sim}^{\mathbf{K}}\right)$ and consider the operators $T$ on $H_{n}^{2}\left(\dot{Z}^{\mathbf{K}}\right)$. The identity

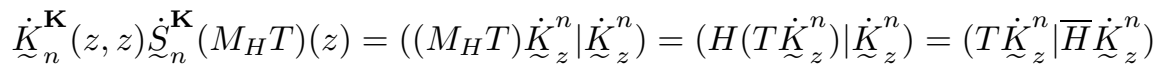

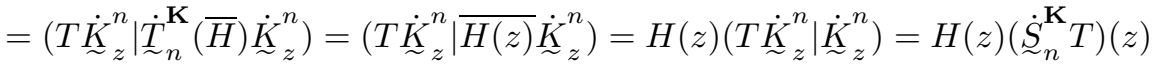

implies

By definition

$$
{\underset{\sim}{S}}_{n}^{\mathbf{K}}\left(M_{H} T\right)=H\left({\underset{\sim}{\dot{S}}}_{n}^{\mathbf{K}} T\right)
$$

$$
\dot{\sim}_{\mathbf{K}}^{\nu}(H \Psi)=\frac{\rho(H \Psi)}{\rho \dot{\sim}_{\mathbf{K}}^{\nu}}=(\rho H) \frac{\rho \Psi}{\rho \dot{\sim}_{\mathbf{K}}^{\nu}}=(\rho H){\underset{\sim}{\mathbf{K}}}_{\mathbf{I}}^{\nu} \Psi .
$$


Putting $\Psi=T \dot{\sim}_{\mathbf{K}}^{\nu}$ it follows that

$$
\begin{aligned}
& \stackrel{\sim}{*}_{\sim}^{\mathbf{K}}\left(H\left(\dot{\sim}_{n}^{\mathbf{K}} T\right)\right)={\underset{\sim}{*}}_{\nu}^{\dot{*}^{\mathbf{K}}}\left(\dot{\sim}_{n}^{\mathbf{K}}\left(M_{H} T\right)\right)=\dot{\sim}_{\mathbf{K}}^{\nu}\left(\left(M_{H} T\right) \dot{\sim}_{\mathbf{K}}^{\nu}\right) \\
& =\dot{\sim}_{\mathbf{K}}^{\nu}\left(H\left(T \dot{\sim}_{\mathbf{K}}^{\nu}\right)\right)=(\rho H) \dot{\sim}_{\mathbf{K}}^{\nu}\left(T \dot{\sim}_{\mathbf{K}}^{\nu}\right)=(\rho H)\left(\stackrel{*}{*}_{\nu}^{\mathbf{K}}{\underset{\sim}{\dot{S}}}_{n}^{\mathbf{K}} T\right) .
\end{aligned}
$$

Putting $F=\dot{\sim}_{n}^{\mathbf{K}} T$ we obtain

$$
\underset{\stackrel{\dot{*}}{\sim}_{\nu}^{\mathbf{K}}}{ }(H F)=(\rho H)\left(\stackrel{\dot{*}}{\sim}_{\nu}^{\mathbf{K}} F\right) .
$$

As a consequence we have for each $j$

$$
\mathcal{N}_{j}(H F)(0)=H(0)\left(\mathcal{N}_{j} F\right)(0),
$$

proving (8.5) for $x=0$. Since $p_{j}$ is real, we have $p_{j}\left(\partial_{\mathbf{K}}\right)=\overline{p_{j}\left(\partial_{\mathbf{K}}\right)}$ and obtain

$$
\begin{gathered}
\mathcal{N}_{j}(\Phi \bar{\Psi})(0)=\Phi(0)\left(\mathcal{N}_{j} \bar{\Psi}\right)(0)=\Phi(0)\left(p_{j}\left(\partial_{\mathbf{K}}\right) \rho \bar{\Psi}\right)(0) \\
=\Phi(0) \overline{p_{j}\left(\partial_{\mathbf{K}}\right) \rho \Psi(0)}=\Phi(0) \overline{p_{j}\left(\partial^{\mathbf{K}}\right) \Psi(0)}=\overline{p_{j}\left(\partial^{\mathbf{K}}\right)}(\Phi \bar{\Psi})(0),
\end{gathered}
$$

showing that

$$
\left(\mathcal{N}_{j} F\right)(0)=\left(\overline{p_{j}\left(\partial^{\mathbf{K}}\right)} F\right)(0)
$$

for all $j$. Since $\left(\overline{p_{j}\left(\partial^{\mathbf{K}}\right)} F\right)(0)$ depends only on the jet of $F$ at 0 , (8.6) remains in force even if $H$ is holomorphic only in some neighborhood of 0 (not necessarily on all of $\underset{\sim}{Z}$ ). Now (8.5) follows by $\dot{G}_{\mathbf{K}}$ invariance, using the fact that the transvections $\dot{\mathfrak{t}}_{x}$ are holomorphic on $\dot{Z}^{\mathrm{K}}$.

Now we specialize to the complex case, where $\nu=n$. For a $K$-invariant "real" sesqui-polynomial $P(z, w)$ on $Z \times \bar{Z}$ as in (5.10), the associated bi-differential operator

$$
P\left(\partial^{\mathbf{C}}\right):=\sum_{i} u_{i}(\partial) \otimes \overline{u_{i}(\partial)}
$$

is $K$-invariant and induces a $\dot{G}$-invariant differential operator $P\left(\dot{\partial}^{\mathbf{C}}\right)$ by

$$
P\left(\dot{\partial}^{\mathbf{C}}\right)\left(f_{1} \otimes f_{2}\right)(z)=P\left(\partial^{\mathbf{C}}\right)\left[\left(f_{1} \circ \dot{\mathfrak{t}}_{z}\right) \otimes\left(f_{2} \circ \dot{\mathfrak{t}}_{z}\right)\right](0,0)
$$

for all $z \in \dot{Z}$. Here $\dot{\mathfrak{t}}_{z} \in \dot{G}$ are the transvections (2.5).

Theorem 8.3. Consider the $K$-invariant "real" sesqui-polynomials $Q_{j}$ on $Z$ in the asymptotic expansion (5.13) of the inverse Berezin operator transform $B_{n}^{-1}$. Then the Toeplitz star-product has the asymptotic expansion as $n \rightarrow+\infty$ :

$$
\left(f_{1} \dot{*} f_{2}\right)(z) \asymp \sum_{j=0}^{\infty} \frac{Q_{j}\left(\dot{\partial}^{\mathbf{C}}\right)\left(f_{1} \otimes f_{2}\right)(z)}{n^{j}}
$$

for $f_{1}, f_{2} \in \mathcal{C}^{\infty}(\dot{Z})$ and $z \in \dot{Z}$. Thus $f_{1} \dot{*} f_{2}$ involves only holomorphic derivatives in the first variable (acting on $f_{1}$ ) and anti-holomorphic derivatives in the second variable (acting on $f_{2}$ ). The 0 -order term is the product map.

Proof. This result is the special case $\mathbf{K}=\mathbf{C}$ of Theorem 8.1 . We will give a direct proof (actually two different proofs) in the compact complex case, which is of current interest. The first proof explains the use of the locality principle, which 
also underlies the general result, in the more familiar star-product setting. By (6.16) we have

$$
\begin{aligned}
& \sum_{j \geq 0} n^{-j} \hat{T}_{n}\left(\overline{\mathcal{L}_{j}\left(f_{1} \otimes f_{2}\right)}\right)=\sum_{j \geq 0} n^{-j} \hat{T}_{n}\left(\mathcal{L}_{j}\left(f_{1} \otimes f_{2}\right)\right)^{*} \\
& =\left[\left(\hat{T}_{n} f_{1}\right)\left(\hat{T}_{n} f_{2}\right)\right]^{*}=\left(\hat{T}_{n} f_{2}\right)^{*}\left(\hat{T}_{n} f_{1}\right)^{*}=\left(\hat{T}_{n} \bar{f}_{2}\right)\left(\hat{T}_{n} \bar{f}_{1}\right)=\sum_{j \geq 0} n^{-j} \hat{T}_{n}\left(\mathcal{L}_{j}\left(\bar{f}_{2} \otimes \bar{f}_{1}\right)\right) .
\end{aligned}
$$

Therefore for every $j \in \mathbf{N}$

$$
\mathcal{L}_{j}\left(\bar{f}_{2} \otimes \bar{f}_{1}\right)=\overline{\mathcal{L}_{j}\left(f_{1} \otimes f_{2}\right)},
$$

and it suffices to prove the first statement concerning the derivatives in the first variable. As a special case of Proposition 7.1 there exists a smooth defining function $-\hat{W}$ for the unit ball $\underset{\sim}{Z}:=\{\|z\|<1\}$ satisfying

$$
\hat{W}(z)=\Delta(z,-z)^{-1}
$$

for $\|z\| \leq 1 / 2$. The associated Hartogs domain

$$
\hat{\mathcal{D}}=\left\{(z, t) \in \underset{\sim}{Z} \times \mathbf{C}:|t|^{2}<\hat{W}(z)\right\}
$$

has the Bergman space $A^{2}(\hat{\mathcal{D}}):=L^{2}\left(\hat{\mathcal{D}}, d \lambda(z, t) \hat{W}(z)^{-\alpha}\right) \cap \operatorname{Hol}(\hat{\mathcal{D}})$ and Toeplitz operators defined analogously to (4.5) as

$$
(\hat{\mathcal{T}} F) \Phi=\hat{\mathcal{P}}(F \Phi), \quad F \in L^{\infty}(\hat{\mathcal{D}})
$$

where $\hat{\mathcal{P}}: L^{2}\left(\hat{\mathcal{D}}, d \lambda(z, t) \hat{W}(z)^{-\alpha}\right) \rightarrow A^{2}(\hat{\mathcal{D}})$ is the orthogonal projection. If $H$ is a bounded holomorphic function these operators satisfy

$$
(\hat{\mathcal{T}} H) \Phi=H \Phi
$$

for all $\Phi \in A^{2}(\hat{\mathcal{D}})$, which implies

$$
(\hat{\mathcal{T}} \bar{H})(\hat{\mathcal{T}} F)=\hat{\mathcal{T}}(\bar{H} F),(\hat{\mathcal{T}} F)(\hat{\mathcal{T}} H)=\hat{\mathcal{T}}(F H)
$$

If $f$ is a bounded function on $\underset{\sim}{Z}$, viewed as a function on $\hat{\mathcal{D}}$ depending only on $z$, then the Toeplitz operator $\hat{\mathcal{T}} f$ on $A^{2}(\hat{\mathcal{D}})$ splits under the decomposition (17.2) into the direct sum

$$
\hat{\mathcal{T}} f=\bigoplus_{n \geq 0} \hat{T}_{n} f
$$

of the Toeplitz operators ${\underset{\sim}{T}}_{n} f$ on $A^{2}\left(\underset{\sim}{Z}, \dot{C}_{n} \hat{W}(z)^{n+p} d \lambda(z)\right)$. Since $\hat{\mathcal{D}}$ is smoothly bounded and strictly pseudoconvex by the conditions imposed on $\hat{W}$, the results in [E1] show that there exists an asymptotic expansion (even in the strong sense of the operator norms (6.17))

$$
\left(\hat{\sim}_{n} f_{1}\right)\left(\hat{\sim}_{n} f_{2}\right) \asymp \hat{\sim}_{n}\left(f_{1} * f_{2}\right)
$$

as $n \rightarrow+\infty$, for some formal power series in $\frac{1}{n}$,

$$
f_{1} * f_{2}:=\sum_{j=0}^{\infty} n^{-j} C_{j}\left(f_{1} \otimes f_{2}\right),
$$

with bi-differential operators $C_{j}$. Then it follows from (8.9) that

$$
\left(\bar{h}_{1} f_{1}\right) *\left(f_{2} h_{2}\right)=\bar{h}_{1}\left(f_{1} * f_{2}\right) h_{2}
$$


for bounded holomorphic functions $h_{1}, h_{2}$ on $\underset{\sim}{Z}$. In particular,

$$
C_{j}\left(\bar{h}_{1} f_{1} \otimes f_{2} h_{2}\right)=\bar{h}_{1} C_{j}\left(f_{1} \otimes f_{2}\right) h_{2} .
$$

Since $\underset{\sim}{Z}$ is bounded (so that the coordinate functions are bounded and holomorphic on $\underset{\sim}{Z}$ ), the bi-differential operators $C_{j}$ necessarily contain only holomorphic derivatives of the first argument and anti-holomorphic derivatives of the second argument (cf. the proof of Proposition 5 in [EU1]). In view of (8.10), the bi-differential operators $C_{j}$ again satisfy

$$
\hat{\sim}_{n}\left[\sum_{n=0}^{\infty} n^{-j} C_{j}\left(f_{1} \otimes f_{2}\right)\right]={\underset{\sim}{S}}_{n}\left[\left(\hat{\sim}_{n} f_{1}\right)\left({\underset{\sim}{\hat{T}}}_{n} f_{2}\right)\right],
$$

where ${\underset{\sim}{\hat{B}}}_{n}$ and $\hat{\sim}_{n}$ are the Berezin transform and the Berezin symbol map, respectively, for $A^{2}\left(\underset{\sim}{Z}, \hat{W}^{n+p}\right)$. Now by Proposition 7.4 and Proposition [7.6. $\left(\hat{\sim}_{n} f\right)(z)$ and ${\underset{\sim}{S}}_{n}\left[\left(\hat{\sim}_{n} f_{1}\right)\left(\hat{T}_{n} f_{2}\right)\right](z)$ have the same asymptotic expansion for $\|z\|<1 / 4$ as (5.12) and (6.15), respectively. It follows that for $\|z\|<1 / 4$, the bi-differential operators $C_{j}$ coincide with our $\mathcal{L}_{j}$, which implies the desired factorization property of $\mathcal{L}_{j}$ for $\|z\|<1 / 4$. By $\hat{G}$-invariance this extends to all $z \in \hat{Z}$.

Second proof. We will now give another proof which does not use the local model but the operator norm estimates (6.17) from [Sch]. (We emphasize that our results concerning the Peter-Weyl decomposition in the next section do not depend on these uniform estimates.) For any polynomial $p \in \mathcal{P}(Z),\left(\hat{T}_{n} f_{2}\right) p$ makes sense as soon as $n \geq \operatorname{deg} p$, and then

$$
\left(\hat{T}_{n} f_{2}\right) p=\hat{P}_{n}\left(f_{2} p\right)=\hat{T}_{n}\left(f_{2} p\right) \mathbf{1}
$$

where 1 stands for the function constant one. For any $f_{1}, f_{2} \in \mathcal{C}_{c}^{\infty}(Z)$ we thus have, by (6.17), for any $N \in \mathbf{N}$ on the one hand

$$
\left|\left(\mathbf{1} \mid\left(\hat{T}_{n} f_{1}\right)\left(\hat{T}_{n}\left(f_{2} p\right)\right) \mathbf{1}\right)_{n}-\sum_{j=0}^{N-1} n^{-j}\left(\mathbf{1} \mid \hat{T}_{n}\left(\mathcal{L}_{j}\left(f_{1} \otimes\left(f_{2} p\right)\right)\right) \mathbf{1}\right)_{n}\right| \leq C_{f_{1}, f_{2} p, N} n^{-N}\|\mathbf{1}\|_{n}^{2},
$$

and on the other hand

$$
\begin{aligned}
& \left|\left(\mathbf{1} \mid\left(\hat{T}_{n} f_{1}\right)\left(\hat{T}_{n}\left(f_{2} p\right)\right) \mathbf{1}\right)_{n}-\sum_{j=0}^{N-1} n^{-j}\left(\mathbf{1} \mid \hat{T}_{n}\left(\mathcal{L}_{j}\left(f_{1} \otimes f_{2}\right) p\right) \mathbf{1}\right)_{n}\right| \\
& =\left|\left(\mathbf{1} \mid\left(\hat{T}_{n} f_{1}\right)\left(\hat{T}_{n} f_{2}\right) p\right)_{n}-\sum_{j=0}^{N-1} n^{-j}\left(\mathbf{1} \mid \hat{T}_{n}\left(\mathcal{L}_{j}\left(f_{1} \otimes f_{2}\right)\right) p\right)_{n}\right| \\
& \leq C_{f_{1}, f_{2}, N} n^{-N}\|p\|_{n}\|\mathbf{1}\|_{n},
\end{aligned}
$$

as $n \rightarrow+\infty$. Since by Proposition [5.4.

$$
\|p\|_{n}^{2}=\hat{C}_{n} \int_{Z}|p|^{2} \hat{K}^{-(n+p)} d \lambda=\hat{B}_{n}|p|^{2}(0) \rightarrow|p(0)|^{2},
$$


both $\|p\|_{n}$ and $\|\mathbf{1}\|_{n}$ stay bounded as $n \rightarrow+\infty$. Subtracting (8.11) and (8.12) we thus get

$$
\sum_{j=0}^{N-1} n^{-j}\left(\mathbf{1} \mid \hat{T}_{n}\left(\mathcal{L}_{j}\left(f_{1} \otimes f_{2} p\right)-\mathcal{L}_{j}\left(f_{1} \otimes f_{2}\right) p\right) \mathbf{1}\right)_{n}=O\left(n^{-N}\right)
$$

or

$$
\sum_{j=0}^{N-1} n^{-j} \hat{B}_{n}\left(\mathcal{L}_{j}\left(f_{1} \otimes f_{2} p\right)-\mathcal{L}_{j}\left(f_{1} \otimes f_{2}\right) p\right)(0)=O\left(n^{-N}\right) .
$$

Taking $N=1$ and again using Proposition 5.4, we see that

$$
\mathcal{L}_{0}\left(f_{1} \otimes f_{2} p\right)(0)=\mathcal{L}_{0}\left(f_{1} \otimes f_{2}\right)(0) p(0)
$$

for any $f_{1}, f_{2} \in \mathcal{C}_{c}^{\infty}(Z)$ and $p \in \mathcal{P}(Z)$. Since $\mathcal{L}_{0}$ is a bi-differential operator, it follows that the expression for $\mathcal{L}_{0}\left(f_{1} \otimes f_{2}\right)(z)$ at $z=0$ involves only antiholomorphic derivatives of $f_{2}$. Since $\hat{G}$ acts by holomorphic mappings, the $\hat{G}$ invariance of $\mathcal{L}_{0}$ implies that the same holds, in fact, at any $z \in \hat{Z}$. In particular, $\mathcal{L}_{0}\left(f_{1} \otimes f_{2} p\right)=\mathcal{L}_{0}\left(f_{1} \otimes f_{2}\right) p$ on $Z$, so that the term $j=0$ in (8.13) vanishes identically. Now taking $N=2$ and multiplying by $n$, Proposition 5.4 again implies that

$$
\mathcal{L}_{1}\left(f_{1} \otimes f_{2} p\right)(0)=\mathcal{L}_{1}\left(f_{1} \otimes f_{2}\right)(0) p(0)
$$

for any $f_{1}, f_{2} \in \mathcal{C}_{c}^{\infty}(Z)$ and $p \in \mathcal{P}(Z)$. Repeating the same argument and continuing by induction, the claim follows.

The validity of the factorization property in Theorem 8.3 is actually asserted in complete generality (i.e. on an arbitrary compact Kähler manifold) in [KS; however, it seems difficult to really pinpoint its proof in that paper. The main tools in $[\mathrm{KS}$ are the stationary phase method and the sophisticated machinery of "generalized Toeplitz operators" of Boutet de Monvel and Guillemin (which so far has no "real" analogue); a "locality" argument as in our first proof of Theorem 8.3 is quite implicit there.

Theorem 8.4. Consider the $K$-invariant "real" sesqui-polynomials $P_{j}$ on $Z$ in the asymptotic expansion (5.12) of the Berezin operator transform $B_{n}$. Then for $f_{1}, f_{2} \in \mathcal{C}^{\infty}(\dot{Z})$ the Berezin star product has an asymptotic expansion as $n \rightarrow+\infty$ :

$$
\left(f_{i} \overline{\dot{*}} f_{2}\right)(z) \asymp \sum_{j=0}^{\infty} \frac{\overline{P_{j}(\dot{\partial} \mathbf{C})}\left(f_{1} \otimes f_{2}\right)(z)}{n^{j}}
$$

for all $z \in \dot{Z}$. This asymptotic expansion involves only anti-holomorphic derivatives acting on $f_{1}$ and holomorphic derivatives acting on $f_{2}$. The 0-order term is the product map.

Proof. Again, this follows from the general result in Theorem 8.2 . For a more direct argument, note that on a bounded domain such as the local model $\underset{\sim}{Z}$, it is an easy consequence of the reproducing property

$$
\phi(z)=\left(\phi \mid{\underset{\sim}{K}}_{z}^{n}\right) \quad \forall z \in \underset{\sim}{Z}, \forall \phi \in A^{2}\left(\underset{\sim}{\dot{Z}}, \dot{C}_{n} \dot{W}^{n-\epsilon p} d \lambda\right)
$$

of the Bergman kernel $\underset{\sim}{\dot{K}}(z, w)=:{\underset{\sim}{\dot{K}}}_{w}^{n}(z)$ that for all $z \in \underset{\sim}{Z}$ and $h$ bounded holomorphic on $\underset{\sim}{Z}$,

$$
\left(\dot{\sim}_{n} \bar{h}\right){\underset{\sim}{\dot{K}}}_{z}^{n}=\overline{h(z)}{\underset{\sim}{\dot{K}}}_{z}^{n} .
$$


It follows that the "local" Berezin transform and Berezin symbol satisfy

$$
\dot{\sim}_{n} h=\dot{\sim}_{n}\left(\dot{\sim}_{n} h\right)=h, \quad \dot{\sim}_{n}\left(\left(\dot{\sim}_{n} h\right) T\right)=h\left(\dot{\sim}_{n} T\right)
$$

for $h$ bounded holomorphic and any operator $T$. This implies for the Berezin star-

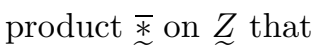

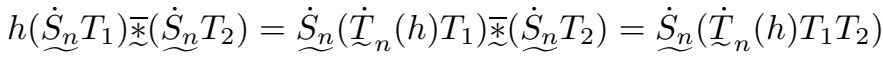

$$
\begin{aligned}
& =h \dot{S}_{n}\left(T_{1} T_{2}\right)=h\left(\left(\dot{S}_{n} T_{1}\right) \underset{\sim}{*}\left(\dot{S}_{n} T_{2}\right)\right),
\end{aligned}
$$

or $(h f) \bar{*} g=h(f \underset{*}{\mp})$ for $h$ bounded holomorphic. Furthermore, by (8.9)

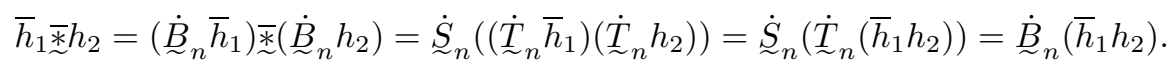

Again using Proposition 7.4 and Proposition [7.6, one sees that $\overline{\dot{*}}$ again has the factorization property, but with the roles of holomorphic and anti-holomorphic functions interchanged. Namely, there exist uniquely determined $\dot{G}$-invariant bidifferential operators $\mathcal{N}_{j}(j \geq 0)$ such that for $f_{1}, f_{2} \in \mathcal{C}^{\infty}(\underset{\sim}{Z})$ we have an asymptotic expansion

$$
f_{1} \underset{n}{\bar{*}} f_{2}=\sum_{j=0}^{\infty} \frac{\mathcal{N}_{j}\left(f_{1} \otimes f_{2}\right)}{n^{j}}
$$

on $\|z\|<1 / 4$, and $\mathcal{N}_{j}\left(f_{1} \otimes f_{2}\right)$ involves only anti-holomorphic derivatives in the first variable (acting on $f_{1}$ ) and only holomorphic derivatives in the second variable (acting on $f_{2}$ ). Furthermore,

$$
\sum_{j=0}^{\infty} \frac{\mathcal{N}_{j}\left(\bar{h}_{1} \otimes h_{2}\right)(z)}{n^{j}}=\dot{B}_{n}\left(\bar{h}_{1} h_{2}\right)(z)
$$

for $\|z\| \leq 1 / 4$ and $h_{1}, h_{2}$ bounded holomorphic on $\underset{\sim}{Z}$. From (5.12) and $\dot{G}$-invariance, the theorem follows.

\section{SpeCtral ANALYSis of the Berezin transform}

For the Peter-Weyl decomposition we need the (generalized) eigenvalues of the Berezin transform, including the real compact case. In the non-compact setting, for real bounded symmetric domains, the spectral analysis of the Berezin transform has been studied by several authors and in full generality in [Z1]. To recall the main result in [Z1], let $\check{G}_{\mathbf{K}}=N A_{\mathbf{K}} K_{\mathbf{K}}$ be the Iwasawa decomposition of $\check{G}_{\mathbf{K}}$, and let $\mathfrak{a}_{\mathbf{K}}$ be the Lie algebra of $A_{\mathbf{K}}$, with dual space $\mathfrak{a}_{\mathbf{K}}^{\prime} \approx \mathbf{R}^{r_{\mathbf{K}}}$. Since $-i d \in K_{\mathbf{K}}$ the (restricted) Weyl group $W$ consists of all even permutations. Thus $\mathcal{P}\left(\mathfrak{a}_{\mathbf{K}}^{\prime}\right)^{W}$ corresponds to the even symmetric polynomials on $\mathbf{R}^{r_{\mathbf{K}}}$.

In all cases, including type (A), the half-sum $\boldsymbol{\rho}=\boldsymbol{\rho}_{\mathbf{K}}$ of positive restricted roots has the components

$$
\rho_{j}=\frac{p_{\mathbf{K}}-1}{2}-\frac{a_{\mathbf{K}}}{2}\left(r_{\mathbf{K}}-j\right)=\frac{p_{\mathbf{K}}+1}{2}-\ell_{\mathbf{K}}+\frac{a_{\mathbf{K}}}{2}(j-1) .
$$

By the Harish-Chandra isomorphism, every $\varphi \in \mathcal{P}\left(\mathfrak{a}_{\mathbf{K}}^{\prime}\right)^{W}$ gives rise to a $\check{G}_{\mathbf{K}}$ invariant differential operator $D_{\varphi}$ on $\check{Z}_{\mathbf{K}}$ such that

$$
D_{\varphi} N_{\boldsymbol{\lambda}+\boldsymbol{\rho}}=\varphi(\boldsymbol{\lambda}) N_{\boldsymbol{\lambda}+\boldsymbol{\rho}}
$$

for the conical functions defined by

$$
N_{\boldsymbol{\mu}}(x)=\Delta_{\boldsymbol{\mu}}\left(\operatorname{Re}\left(\hat{\mathfrak{t}}_{e}(x)\right)\right) .
$$


Here $\Delta_{\boldsymbol{\mu}}$ are the usual power functions on $Z_{\mathbf{K}}^{+}, \hat{\mathfrak{t}}_{e}$ is the Cayley transformation which maps $\breve{Z}_{\mathbf{K}}$ onto the Siegel domain associated with $e$ and Re denotes the generalized "real-part" mapping [L] for the Siegel domain. The analysis above generalizes to $\check{G}_{\mathbf{K}}$-invariant pseudo-differential operators such as the Berezin transform, and we obtain

$$
\check{\beta}_{\mathbf{K}}^{\nu} N_{\boldsymbol{\lambda}+\boldsymbol{\rho}}=\check{b}_{\mathbf{K}}^{\nu}(\boldsymbol{\lambda}) N_{\boldsymbol{\lambda}+\boldsymbol{\rho}}
$$

for a unique even symmetric function $\check{b}_{\mathbf{K}}^{\nu}$.

Theorem 9.1 ([Z1, Theorem A]). For all $\boldsymbol{\mu} \in \mathbf{N}_{+}^{r_{\mathbf{K}}}$ let $\boldsymbol{\mu}^{*}=\left(\mu_{r_{\mathbf{K}}}, \ldots, \mu_{1}\right)$. Then

$$
\begin{gathered}
\check{b}_{\mathbf{K}}^{\nu}(\boldsymbol{\rho}+\boldsymbol{\mu})=\prod_{j=1}^{r_{\mathbf{K}}} \frac{\Gamma\left(\nu+\frac{1-p_{\mathbf{K}}}{2}+\rho_{j}+\mu_{j}\right) \Gamma\left(\nu+\frac{1-p_{\mathbf{K}}}{2}-\rho_{j}-\mu_{j}\right)}{\Gamma\left(\nu+\frac{1-p_{\mathbf{K}}}{2}+\rho_{j}\right) \Gamma\left(\nu+\frac{1-p_{\mathbf{K}}}{2}-\rho_{j}\right)} \\
=\frac{\Gamma_{\Omega_{\mathbf{K}}}\left(\nu+\boldsymbol{\mu}^{*}\right) \Gamma_{\Omega_{\mathbf{K}}}\left(\nu+\ell_{\mathbf{K}}-p_{\mathbf{K}}-\boldsymbol{\mu}\right)}{\Gamma_{\Omega_{\mathbf{K}}}(\nu) \Gamma_{\Omega_{\mathbf{K}}}\left(\nu+\ell_{\mathbf{K}}-p_{\mathbf{K}}\right)}=(-1)^{\boldsymbol{\mu}} \frac{(\nu)_{\boldsymbol{\mu}^{*}}}{\left(p_{\mathbf{K}}-\nu\right)_{\boldsymbol{\mu}^{*}}} .
\end{gathered}
$$

Proof. The first formula is just a restatement of [Z1, Theorem A]

$$
\check{b}_{\mathbf{K}}^{\nu}(\boldsymbol{\lambda})=\prod_{j=1}^{r_{\mathbf{K}}} \frac{\Gamma\left(\nu+\frac{1-p_{\mathbf{K}}}{2}+\lambda_{j}\right) \Gamma\left(\nu+\frac{1-p_{\mathbf{K}}}{2}-\lambda_{j}\right)}{\Gamma\left(\nu+\frac{1-p_{\mathbf{K}}}{2}+\rho_{j}\right) \Gamma\left(\nu+\frac{1-p_{\mathbf{K}}}{2}-\rho_{j}\right)},
$$

by putting $\boldsymbol{\lambda}=\boldsymbol{\rho}+\boldsymbol{\mu}$. Since (9.1) implies

$$
\frac{1-p_{\mathbf{K}}}{2}-\rho_{j}=1-p_{\mathbf{K}}+\frac{a_{\mathbf{K}}}{2}\left(r_{\mathbf{K}}-j\right)=\ell_{\mathbf{K}}-p_{\mathbf{K}}-\frac{a_{\mathbf{K}}}{2}(j-1)
$$

the second formula follows from

$$
\begin{gathered}
\check{b}_{\mathbf{K}}^{\nu}(\boldsymbol{\rho}+\boldsymbol{\mu})=\prod_{j=1}^{r_{\mathbf{K}}} \frac{\Gamma\left(\nu+\frac{1-p_{\mathbf{K}}}{2}+\rho_{j}+\mu_{j}\right) \Gamma\left(\nu+\frac{1-p_{\mathbf{K}}}{2}-\rho_{j}-\mu_{j}\right)}{\Gamma\left(\nu+\frac{1-p_{\mathbf{K}}}{2}+\rho_{j}\right) \Gamma\left(\nu+\frac{1-p_{\mathbf{K}}}{2}-\rho_{j}\right)} \\
=\prod_{j=1}^{r_{\mathbf{K}}} \frac{\Gamma\left(\nu-\frac{a_{\mathbf{K}}}{2}\left(r_{\mathbf{K}}-j\right)+\mu_{j}\right) \Gamma\left(\nu+\ell_{\mathbf{K}}-p_{\mathbf{K}}-\frac{a_{\mathbf{K}}}{2}(j-1)-\mu_{j}\right)}{\Gamma\left(\nu-\frac{a_{\mathbf{K}}}{2}\left(r_{\mathbf{K}}-j\right)\right) \Gamma\left(\nu+\ell_{\mathbf{K}}-p_{\mathbf{K}}-\frac{a_{\mathbf{K}}}{2}(j-1)\right)} \\
=\frac{\Gamma_{\Omega_{\mathbf{K}}}\left(\nu+\boldsymbol{\mu}^{*}\right) \Gamma_{\Omega_{\mathbf{K}}}\left(\nu+\ell_{\mathbf{K}}-p_{\mathbf{K}}-\boldsymbol{\mu}\right)}{\Gamma_{\Omega_{\mathbf{K}}}(\nu) \Gamma_{\Omega_{\mathbf{K}}}\left(\nu+\ell_{\mathbf{K}}-p_{\mathbf{K}}\right)} .
\end{gathered}
$$

Putting $x=\nu+\ell_{\mathbf{K}}-p_{\mathbf{K}}$ and applying (3.16) we obtain the assertion

$$
(-1)^{\boldsymbol{\mu}} \frac{\Gamma_{\Omega_{\mathbf{K}}}\left(\nu+\ell_{\mathbf{K}}-p_{\mathbf{K}}\right)}{\Gamma_{\Omega_{\mathbf{K}}}\left(\nu+\ell_{\mathbf{K}}-p_{\mathbf{K}}-\boldsymbol{\mu}\right)}=\frac{\Gamma_{\Omega_{\mathbf{K}}}\left(p_{\mathbf{K}}-\nu+\boldsymbol{\mu}^{*}\right)}{\Gamma_{\Omega_{\mathbf{K}}}\left(p_{\mathbf{K}}-\nu\right)}=\left(p_{\mathbf{K}}-\nu\right)_{\boldsymbol{\mu}^{*}} .
$$

In the complex case, where $\nu=n$ and $\boldsymbol{\mu}=\boldsymbol{m}$ has length $r$, we have

$$
\rho_{j}:=\frac{p-1}{2}-\frac{a}{2}(r-j)=\frac{p+1}{2}-\ell_{\mathbf{C}}+\frac{a}{2}(j-1)
$$

and obtain the formula $\mathrm{UU}$

$$
\begin{gathered}
\check{b}_{\mathbf{C}}^{n}(\boldsymbol{\rho}+\boldsymbol{m})=\prod_{j=1}^{r} \frac{\Gamma\left(n+\frac{1-p}{2}+\rho_{j}+m_{j}\right) \Gamma\left(n+\frac{1-p}{2}-\rho_{j}-m_{j}\right)}{\Gamma\left(n+\frac{1-p}{2}+\rho_{j}\right) \Gamma\left(n+\frac{1-p}{2}-\rho_{j}\right)} \\
=\frac{\Gamma_{\Omega}\left(n+\boldsymbol{m}^{*}\right) \Gamma_{\Omega}\left(n+\ell_{\mathbf{C}}-p-\boldsymbol{m}\right)}{\Gamma_{\Omega}(n) \Gamma_{\Omega}\left(n+\ell_{\mathbf{C}}-p\right)}=(-1)^{\boldsymbol{m}} \frac{(n)_{\boldsymbol{m}^{*}}}{(p-n)_{\boldsymbol{m}^{*}}} .
\end{gathered}
$$


Since $\hat{Z}_{\mathbf{K}}=\hat{G}_{\mathbf{K}} / K_{\mathbf{K}}$ is a compact symmetric space, the space $L^{2}\left(\hat{Z}_{\mathbf{K}}\right)$, for the normalized Haar measure (3.17), has a multiplicity-free "Peter-Weyl" decomposi-

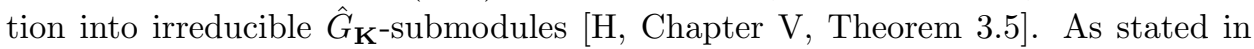
[Z2, Lemma 3.1], this "Peter-Weyl" decomposition can be described in a uniform manner:

$$
L^{2}\left(\hat{Z}_{\mathbf{K}}\right)=\sum_{\boldsymbol{\mu}} L_{\boldsymbol{\mu}}^{2}\left(\hat{Z}_{\mathbf{K}}\right)
$$

where $\boldsymbol{\mu}$ runs over all partitions of length $r_{\mathbf{K}}$, and $L_{\boldsymbol{\mu}}^{2}\left(\hat{Z}_{\mathbf{K}}\right)$ is the (finite dimensional) $\hat{G}_{\mathbf{K}}$-submodule generated by the spherical function $\hat{\Phi}_{\boldsymbol{\mu}}$.

Example 9.2. In the rank 1 case $\hat{Z}_{\mathbf{C}}=\mathbf{C P}^{1} \approx \mathbf{S}^{2}$, the corresponding spherical function is

$$
\hat{\Phi}_{m}(z)=G_{m}^{\mathbf{K}}\left(\frac{1-|z|^{2}}{1+|z|^{2}}\right),
$$

where $G_{m}^{\mathbf{K}}$ is the $m$-th Gegenbauer polynomial.

The Berezin transform $\hat{\beta}_{\mathbf{K}}^{\nu}$, regarded as a densely defined operator on $L^{2}\left(\hat{Z}_{\mathbf{K}}\right)$, is invariant under $\hat{G}_{\mathbf{K}}$ and is therefore diagonalized under the decomposition (9.3). The corresponding eigenvalues $\hat{b}_{\mathbf{K}}^{\nu}(\boldsymbol{\mu})$ are defined by

$$
\hat{\beta}_{\mathbf{K}}^{\nu} \hat{\Phi}_{\boldsymbol{\mu}}=\hat{b}_{\mathbf{K}}^{\nu}(\boldsymbol{\mu}) \hat{\Phi}_{\boldsymbol{\mu}}
$$

The main result of this section is

Theorem 9.3.

$$
\begin{gathered}
\hat{b}_{\mathbf{K}}^{\nu}(\boldsymbol{\mu})=\prod_{j=1}^{r_{\mathbf{K}}} \frac{\Gamma\left(\nu+\frac{p_{\mathbf{K}}+1}{2}+\rho_{j}\right) \Gamma\left(\nu+\frac{p_{\mathbf{K}}+1}{2}-\rho_{j}\right)}{\Gamma\left(\nu+\frac{p_{\mathbf{K}}+1}{2}+\rho_{j}+\mu_{j}\right) \Gamma\left(\nu+\frac{p_{\mathbf{K}}+1}{2}-\rho_{j}-\mu_{j}\right)} \\
=\frac{\Gamma_{\Omega_{\mathbf{K}}}\left(\nu+\ell_{\mathbf{K}}\right) \Gamma_{\Omega_{\mathbf{K}}}\left(\nu+p_{\mathbf{K}}\right)}{\Gamma_{\Omega_{\mathbf{K}}}\left(\nu+\ell_{\mathbf{K}}-\boldsymbol{\mu}\right) \Gamma_{\Omega_{\mathbf{K}}}\left(\nu+p_{\mathbf{K}}+\boldsymbol{\mu}^{*}\right)}=(-1)^{\boldsymbol{\mu}} \frac{(-\nu)_{\boldsymbol{\mu}^{*}}}{\left(p_{\mathbf{K}}+\nu\right)_{\boldsymbol{\mu}^{*}}}=\check{b}_{\mathbf{K}}^{-\nu}(\boldsymbol{\rho}+\boldsymbol{\mu}) .
\end{gathered}
$$

The proof requires several steps, similar to those in [Z2], but with some simplifications. Put

$$
\mathbf{1}:=(1, \ldots, 1)
$$

of length $r_{\mathbf{K}}$.

\section{Proposition 9.4.}

$$
\frac{\check{C}_{\mathbf{K}}^{1-\nu}}{\check{C}_{\mathbf{K}}^{-\nu}}=\frac{\hat{C}_{\mathbf{K}}^{\nu-1}}{\hat{C}_{\mathbf{K}}^{\nu}}
$$

Proof. We have

$$
\frac{\check{C}_{\mathbf{K}}^{1+\nu}}{\check{C}_{\mathbf{K}}^{\nu}}=\frac{\Gamma_{\Omega_{\mathbf{K}}}\left(\nu+1+\ell_{\mathbf{K}}-p_{\mathbf{K}}+\frac{d}{2 r_{\mathbf{K}}}\right) \Gamma_{\Omega_{\mathbf{K}}}\left(\nu+\ell_{\mathbf{K}}-p_{\mathbf{K}}\right)}{\Gamma_{\Omega_{\mathbf{K}}}\left(\nu+\ell_{\mathbf{K}}-p_{\mathbf{K}}+\frac{d}{2 r_{\mathbf{K}}}\right) \Gamma_{\Omega_{\mathbf{K}}}\left(\nu+1+\ell_{\mathbf{K}}-p_{\mathbf{K}}\right)}=\frac{\left(\nu+\ell_{\mathbf{K}}-p_{\mathbf{K}}+\frac{d}{2 r_{\mathbf{K}}}\right)_{\mathbf{1}}}{\left(\nu+\ell_{\mathbf{K}}-p_{\mathbf{K}}\right)_{\mathbf{1}}}
$$

and

$$
\frac{\hat{C}_{\mathbf{K}}^{\nu-1}}{\hat{C}_{\mathbf{K}}^{\nu}}=\frac{\Gamma_{\Omega_{\mathbf{K}}}\left(\nu-1+p_{\mathbf{K}}\right) \Gamma_{\Omega_{\mathbf{K}}}\left(\nu+p_{\mathbf{K}}-\frac{d}{2 r_{\mathbf{K}}}\right)}{\Gamma_{\Omega_{\mathbf{K}}}\left(\nu+p_{\mathbf{K}}\right) \Gamma_{\Omega_{\mathbf{K}}}\left(\nu-1+p_{\mathbf{K}}-\frac{d}{2 r_{\mathbf{K}}}\right)}=\frac{\left(\nu-1+p_{\mathbf{K}}-\frac{d}{2 r_{\mathbf{K}}}\right)_{\mathbf{1}}}{\left(\nu-1+p_{\mathbf{K}}\right)_{\mathbf{1}}} .
$$

Since

$$
(-x)_{\mathbf{1}}=(-1)^{r_{\mathbf{K}}}\left(x+\frac{a_{\mathbf{K}}}{2}\left(r_{\mathbf{K}}-1\right)\right)_{\mathbf{1}}=(-1)^{r_{\mathbf{K}}}\left(x+\ell_{\mathbf{K}}-1\right)_{\mathbf{1}}
$$


we obtain

$$
\frac{\check{C}_{\mathbf{K}}^{1-\nu}}{\check{C}_{\mathbf{K}}^{-\nu}}=\frac{\left(-\nu+\ell_{\mathbf{K}}-p_{\mathbf{K}}+\frac{d}{2 r_{\mathbf{K}}}\right)_{\mathbf{1}}}{\left(-\nu+\ell_{\mathbf{K}}-p_{\mathbf{K}}\right)_{\mathbf{1}}}=\frac{\left(\nu-1+p_{\mathbf{K}}-\frac{d}{2 r_{\mathbf{K}}}\right)_{\mathbf{1}}}{\left(\nu-1+p_{\mathbf{K}}\right)_{\mathbf{1}}}=\frac{\hat{C}_{\mathbf{K}}^{\nu-1}}{\hat{C}_{\mathbf{K}}^{\nu}} .
$$

For a real-analytic function $f$ on a neighborhood of $0 \in Z_{\mathbf{K}}$ let $f^{\mathbf{C}}$ be the holomorphic extension to a neighborhood of $0 \in Z^{\mathbf{K}}$ and consider the function

$$
(f \circ i)(x):=f^{\mathbf{C}}(i x)
$$

for $x \in Z_{\mathbf{K}}$ near 0 .

Proposition 9.5. Let $q$ be a $K_{\mathbf{K}}$-invariant polynomial on $Z_{\mathbf{K}}$. Then $q\left(\check{\partial}_{\mathbf{K}}\right) f=F$ implies

$$
(q \circ i)\left(\hat{\partial}_{\mathbf{K}}\right)(f \circ i)=F \circ i
$$

for the associated invariant differential operators defined in (5.5).

Proof. Let $p(x)=(x \mid a)^{n}$, where $n \in \mathbf{N}$ and $a \in Z^{\mathbf{K}}$. Then

$$
\left(p\left(\partial_{\mathbf{K}}\right) \varphi\right)(0)=\varphi^{(n)}(0) a \otimes \cdots \otimes a .
$$

Since $(p \circ i)(x)=(i x \mid a)^{n}=(x \mid-i a)^{n}$, it follows that

$$
\begin{gathered}
\left((p \circ i)\left(\partial_{\mathbf{K}}\right) \varphi\right)(0)=\varphi^{(n)}(0) \\
=(-i a) \otimes \cdots \otimes(-i a)=\left(\varphi \circ i^{-1}\right)^{(n)}(0) a \otimes \cdots \otimes a \\
=\left(p\left(\partial_{\mathbf{K}}\right)\left(\varphi \circ i^{-1}\right)\right)(0) .
\end{gathered}
$$

Expressing $q$ as a sum of powers, we obtain

$$
\left((q \circ i)\left(\partial_{\mathbf{K}}\right) \varphi\right)(0)=\left(q\left(\partial_{\mathbf{K}}\right)\left(\varphi \circ i^{-1}\right)\right)(0) .
$$

Now assume the identity $q\left(\check{\partial}_{\mathbf{K}}\right) f=F$ holds. Then

$$
F(g(0))=\left(q\left(\check{\partial}_{\mathbf{K}}\right) f\right)(g(0))=q\left(\partial_{\mathbf{K}}\right)(f \circ g)(0)
$$

for all $g \in \check{G}_{\mathbf{K}}$ near $i d$. By analytic continuation, this implies

$$
F^{\mathbf{C}}(h(0))=q\left(\partial_{\mathbf{K}}\right)\left(f^{\mathbf{C}} \circ h\right)(0)
$$

for all $h \in \check{G}_{\mathbf{K}}^{\mathbf{C}}$ near $i d$. Now let $\gamma \in \hat{G}_{\mathbf{K}}$ near $i d$. Then $A d(i) \gamma=i \circ \gamma \circ i^{-1} \in G_{\mathbf{K}}^{*} \subset$ $\check{G}_{\mathbf{K}}^{\mathbf{C}}$. Therefore

$$
\begin{gathered}
(F \circ i)(\gamma(0))=F^{\mathbf{C}}((A d(i) \gamma)(0))=q\left(\partial_{\mathbf{K}}\right)\left(f^{\mathbf{C}} \circ \operatorname{Ad}(i) \gamma\right)(0) \\
=q\left(\partial_{\mathbf{K}}\right)\left((f \circ i) \circ \gamma \circ i^{-1}\right)(0)=\left((q \circ i)\left(\partial_{\mathbf{K}}\right)((f \circ i) \circ \gamma)\right)(0)=\left((q \circ i)\left(\hat{\partial}_{\mathbf{K}}\right)(f \circ i)\right)(\gamma(0)) .
\end{gathered}
$$

The function

$$
\begin{gathered}
\psi_{\nu}(\boldsymbol{\lambda}):=\frac{\check{b}_{\mathbf{K}}^{\nu}(\boldsymbol{\lambda})}{\check{b}_{\mathbf{K}}^{\nu-1}(\boldsymbol{\lambda})} \\
=\frac{\Gamma_{\Omega_{\mathbf{K}}}\left(\nu+\boldsymbol{\mu}^{*}\right) \Gamma_{\Omega_{\mathbf{K}}}\left(\nu+\ell_{\mathbf{K}}-p_{\mathbf{K}}-\boldsymbol{\mu}\right) \Gamma_{\Omega_{\mathbf{K}}}(\nu-1) \Gamma_{\Omega_{\mathbf{K}}}\left(\nu-1+\ell_{\mathbf{K}}-p_{\mathbf{K}}\right)}{\Gamma_{\Omega_{\mathbf{K}}}\left(\nu-1+\boldsymbol{\mu}^{*}\right) \Gamma_{\Omega_{\mathbf{K}}}\left(\nu-1+\ell_{\mathbf{K}}-p_{\mathbf{K}}-\boldsymbol{\mu}\right) \Gamma_{\Omega_{\mathbf{K}}}(\nu) \Gamma_{\Omega_{\mathbf{K}}}\left(\nu+\ell_{\mathbf{K}}-p_{\mathbf{K}}\right)} \\
=\frac{\left(\nu-1+\boldsymbol{\mu}^{*}\right)_{\mathbf{1}}\left(\nu-1+\ell_{\mathbf{K}}-p_{\mathbf{K}}-\boldsymbol{\mu}\right)_{\mathbf{1}}}{(\nu-1)_{\mathbf{1}}\left(\nu-1+\ell_{\mathbf{K}}-p_{\mathbf{K}}\right)_{\mathbf{1}}} \\
=\frac{\prod_{j=1}^{r_{\mathbf{K}}}\left(\nu-\frac{p_{\mathbf{K}}+1}{2}+\lambda_{j}\right)\left(\nu-\frac{1+p}{2}-\lambda_{j}\right)}{(\nu-1)_{\mathbf{1}}\left(\nu-1+\ell_{\mathbf{K}}-p_{\mathbf{K}}\right)_{\mathbf{1}}}=\frac{\prod_{j=1}^{r_{1}}\left(\left(\nu-\frac{p_{\mathbf{K}}+1}{2}\right)^{2}-\lambda_{j}^{2}\right)}{(\nu-1)_{\mathbf{1}}\left(\nu-1+\ell_{\mathbf{K}}-p_{\mathbf{K}}\right)_{\mathbf{1}}}
\end{gathered}
$$


is an even symmetric polynomial in $\boldsymbol{\lambda}=\boldsymbol{\rho}+\boldsymbol{\mu}$. Let $p_{\nu}$ be the unique $K_{\mathbf{K}}$-invariant polynomial on $Z_{\mathbf{K}}$ such that $p_{\nu}\left(\check{\partial}_{\mathbf{K}}\right)=D_{\psi_{\nu}}$. Then the identity $\check{b}_{\mathbf{K}}^{\nu}=\psi_{\nu} \check{b}_{\mathbf{K}}^{\nu-1}$ implies

$$
\check{b}_{\mathbf{K}}^{\nu}=D_{\psi_{\nu}} \check{b}_{\mathbf{K}}^{\nu-1}=p_{\nu}\left(\check{\partial}_{\mathbf{K}}\right) \check{b}_{\mathbf{K}}^{\nu-1} \text {. }
$$

Using the function

$$
\dot{\eta}(x):=\Delta(x, \epsilon x)^{r_{\mathbf{K}} / r}=\dot{K}(x, x)^{-\epsilon r_{\mathbf{K}} / r}
$$

on $\dot{Z}_{\mathbf{K}} \subset \dot{Z}$ we write the Berezin transform for $\epsilon= \pm 1$ as

$$
\dot{\beta}_{\mathbf{K}}^{\nu} f=\dot{C}_{\mathbf{K}}^{\nu} \int_{\dot{Z}_{\mathbf{K}}} \frac{d \lambda(y)}{\dot{\eta}(y)^{p_{\mathbf{K}}}} f(y) \dot{\eta}^{\epsilon \nu} \circ \dot{\mathfrak{t}}_{y}^{-1}
$$

using (2.3), 3.15) and $\dot{G}_{\mathbf{K}}$-invariance. Since $p_{\nu}\left(\check{\partial}_{\mathbf{K}}\right)$ is $\check{G}_{\mathbf{K}}$-invariant, we obtain from (9.7)

$$
\begin{gathered}
\check{C}_{\mathbf{K}}^{\nu-1} \int_{\check{Z}_{\mathbf{K}}} \frac{d \lambda(y)}{\check{\eta}(y)^{p_{\mathbf{K}}}} f(y)\left(p_{\nu}\left(\check{\partial}_{\mathbf{K}}\right) \check{\eta}^{\nu-1}\right) \circ \check{\mathfrak{t}}_{y}^{-1}=p_{\nu}\left(\check{\partial}_{\mathbf{K}}\right) \check{\beta}_{\mathbf{K}}^{\nu-1} f \\
=\check{\beta}_{\mathbf{K}}^{\nu} f=\check{C}_{\mathbf{K}}^{\nu} \int_{\check{Z}_{\mathbf{K}}} \frac{d \lambda(y)}{\check{\eta}(y)^{p_{\mathbf{K}}}} f(y) \check{\eta}^{\nu} \circ \check{\mathfrak{t}}_{y}^{-1} .
\end{gathered}
$$

Since $f$ is arbitrary, we conclude that

$$
\check{C}_{\mathbf{K}}^{\nu-1} p_{\nu}\left(\check{\partial}_{\mathbf{K}}\right) \check{\eta}^{\nu-1}=\check{C}_{\mathbf{K}}^{\nu} \check{\eta}^{\nu}
$$

Since

$$
\check{\eta}^{\mathbf{C}}(i x)=\check{K}^{\mathbf{K}}(i x,-i x)^{r_{\mathbf{K}} / \kappa r}=\check{K}^{\mathbf{K}}(x,-x)^{r_{\mathbf{K}} / \kappa r}=\hat{K}^{\mathbf{K}}(x, x)^{-r_{\mathbf{K}} / \kappa r}=\hat{\eta}(x)
$$

we have $\check{\eta} \circ i=\hat{\eta}$. A similar relation holds for the powers, and (9.9) together with Proposition 9.5 implies

$$
\check{C}_{\mathbf{K}}^{\nu-1}\left(p_{\nu} \circ i\right)\left(\hat{\partial}_{\mathbf{K}}\right) \hat{\eta}^{\nu-1}=\check{C}_{\mathbf{K}}^{\nu} \hat{\eta}^{\nu}
$$

Replacing $\nu$ by $-\nu$ and using the reciprocity (9.5) we obtain

$$
\hat{C}_{\mathbf{K}}^{\nu+1}\left(p_{-\nu} \circ i\right)\left(\hat{\partial}_{\mathbf{K}}\right) \hat{\eta}^{-\nu-1}=\hat{C}_{\mathbf{K}}^{\nu} \hat{\eta}^{-\nu} .
$$

Applying (9.8) for $\epsilon=-1$ we obtain

$$
\left(p_{-\nu} \circ i\right)\left(\hat{\partial}_{\mathbf{K}}\right) \circ \hat{\beta}_{\mathbf{K}}^{\nu+1}=\hat{\beta}_{\mathbf{K}}^{\nu} .
$$

Now consider the spherical functions

$$
\check{\Phi}_{\boldsymbol{\lambda}}=\int_{K_{\mathrm{K}}} d k N_{\boldsymbol{\lambda}+\boldsymbol{\rho}} \circ k
$$

on $\check{Z}_{\mathbf{K}}$ which also satisfy

$$
\check{\beta}_{\mathbf{K}}^{\nu} \check{\Phi}_{\boldsymbol{\lambda}}=\check{b}_{\mathbf{K}}^{\nu}(\boldsymbol{\lambda}) \check{\Phi}_{\boldsymbol{\lambda}}
$$

Proposition 9.6.

$$
\hat{\Phi}_{\boldsymbol{\mu}}=\check{\Phi}_{\boldsymbol{\rho}+\boldsymbol{\mu}} \circ i \text {. }
$$

Proof. By Harish-Chandra's formula (written with $\boldsymbol{\lambda}$ instead of $i \boldsymbol{\lambda}$ ) we have

$$
\check{\Phi}_{\boldsymbol{\lambda}}(g(0))=\int_{K_{\mathbf{K}}} d k A(k g)^{\boldsymbol{\rho}+\boldsymbol{\lambda}}
$$

for all $g \in \check{G}_{\mathbf{K}}$, where $A(g) \in A_{\mathbf{K}}$ denotes the Iwasawa component of $g$. Therefore

$$
\check{\Phi}_{\boldsymbol{\lambda}}(g(0))=\check{\Phi}_{-\boldsymbol{\lambda}}\left(g^{-1}(0)\right)=\int_{K_{\mathbf{K}}} d k A\left(k g^{-1}\right)^{\boldsymbol{\rho}-\boldsymbol{\lambda}} .
$$


We have

$$
i \hat{Z}_{\mathbf{K}} \approx G_{\mathbf{K}}^{*} / K_{\mathbf{K}}
$$

for the compact subgroup $G_{\mathbf{K}}^{*}=A d(i) \hat{G}_{\mathbf{K}} \subset \check{G}_{\mathbf{K}}^{\mathbf{C}}$ with Lie algebra $\mathfrak{g}_{\mathbf{K}}^{*}=\mathfrak{k}_{\mathbf{K}} \oplus i \check{\mathfrak{p}}_{\mathbf{K}}$, where $\check{\mathfrak{g}}_{\mathbf{K}}=\mathfrak{k}_{\mathbf{K}} \oplus \check{\mathfrak{p}}_{\mathbf{K}}$ is the Lie algebra of $\check{G}_{\mathbf{K}}$. If $\boldsymbol{\mu}$ is an integer partition, viewed as a restricted highest weight on $G_{\mathbf{K}}^{*}$, the associated spherical function $\Phi_{\boldsymbol{\mu}}^{*}$ has a holomorphic extension satisfying

$$
\Phi_{\boldsymbol{\mu}}^{*}(g(0))=\int_{K_{\mathbf{K}}} d k A\left(k g^{-1}\right)^{-\boldsymbol{\mu}}=\check{\Phi}_{\boldsymbol{\rho}+\boldsymbol{\mu}}(g(0))
$$

for $g \in \check{G}_{\mathbf{K}}$ near $i d$. Thus

$$
\Phi_{\boldsymbol{\mu}}^{*}(x)=\check{\Phi}_{\rho+\boldsymbol{\mu}}(x)
$$

or, equivalently,

$$
\hat{\Phi}_{\boldsymbol{\mu}}(x)=\Phi_{\boldsymbol{\mu}}^{*}(i x)=\check{\Phi}_{\boldsymbol{\rho}+\boldsymbol{\mu}}^{\mathbf{C}}(i x)
$$

for all $x \in Z_{\mathbf{K}}$ near 0 .

Writing (9.6) in terms of elementary symmetric functions in $\boldsymbol{\lambda}=\boldsymbol{\rho}+\boldsymbol{\mu}$, with coefficients depending on $\nu$, it is clear that $p_{-\nu}$ corresponds to $\psi_{-\nu}$. Therefore

$$
p_{-\nu}\left(\check{\partial}_{\mathbf{K}}\right) \check{\Phi}_{\boldsymbol{\rho}+\boldsymbol{\mu}}=D_{\psi_{-\nu}} \check{\Phi}_{\boldsymbol{\rho}+\boldsymbol{\mu}}=\psi_{-\nu}(\boldsymbol{\rho}+\boldsymbol{\mu}) \check{\Phi}_{\boldsymbol{\rho}+\boldsymbol{\mu}}
$$

Applying Proposition 9.5 and Proposition 9.6 this implies

$$
\left(p_{-\nu} \circ i\right)\left(\hat{\partial}_{\mathbf{K}}\right) \hat{\Phi}_{\boldsymbol{\mu}}=\psi_{-\nu}(\boldsymbol{\rho}+\boldsymbol{\mu}) \hat{\Phi}_{\boldsymbol{\mu}} .
$$

Applying (9.10) to $\hat{\Phi}_{\boldsymbol{\mu}}$ it follows that

$$
\begin{gathered}
\hat{b}_{\mathbf{K}}^{\nu}(\boldsymbol{\mu}) \hat{\Phi}_{\boldsymbol{\mu}}=\hat{\beta}_{\mathbf{K}}^{\nu} \hat{\Phi}_{\boldsymbol{\mu}}=\left(p_{-\nu} \circ i\right)\left(\hat{\partial}_{\mathbf{K}}\right) \circ \hat{\beta}_{\mathbf{K}}^{\nu+1} \hat{\Phi}_{\boldsymbol{\mu}} \\
=\hat{b}_{\mathbf{K}}^{\nu+1}(\boldsymbol{\mu})\left(p_{-\nu} \circ i\right)\left(\hat{\partial}_{\mathbf{K}}\right) \hat{\Phi}_{\boldsymbol{\mu}}=\hat{b}_{\mathbf{K}}^{\nu+1}(\boldsymbol{\mu}) \psi_{-\nu}(\boldsymbol{\rho}+\boldsymbol{\mu}) \hat{\Phi}_{\boldsymbol{\mu}} .
\end{gathered}
$$

Thus we obtain the recursion formula

$$
\begin{gathered}
\frac{\hat{b}_{\mathbf{K}}^{\nu}(\boldsymbol{\mu})}{\hat{b}_{\mathbf{K}}^{\nu+1}(\boldsymbol{\mu})}=\psi_{-\nu}(\boldsymbol{\rho}+\boldsymbol{\mu})=\frac{\prod_{j=1}^{r_{\mathbf{K}}}\left(\left(\nu+\frac{p_{\mathbf{K}}+1}{2}\right)^{2}-\left(\mu_{j}+\rho_{j}\right)^{2}\right)}{\left(\nu+\ell_{\mathbf{K}}\right)_{\mathbf{1}}\left(\nu+p_{\mathbf{K}}\right)_{\mathbf{1}}} \\
=\frac{\left(\nu+\ell_{\mathbf{K}}-\boldsymbol{\mu}\right)_{\mathbf{1}}\left(\nu+p_{\mathbf{K}}+\boldsymbol{\mu}^{*}\right)_{\mathbf{1}}}{\left(\nu+\ell_{\mathbf{K}}\right)_{\mathbf{1}}\left(\nu+p_{\mathbf{K}}\right)_{\mathbf{1}}}
\end{gathered}
$$

Iterating, we obtain

$$
\frac{\hat{b}_{\mathbf{K}}^{\nu}(\boldsymbol{\mu})}{\hat{b}_{\mathbf{K}}^{N+1+\nu}(\boldsymbol{\mu})}=\prod_{k=0}^{N} \frac{\left(\nu+k+\ell_{\mathbf{K}}-\boldsymbol{\mu}\right)_{\mathbf{1}}\left(\nu+k+p_{\mathbf{K}}+\boldsymbol{\mu}^{*}\right)_{\mathbf{1}}}{\left(\nu+k+\ell_{\mathbf{K}}\right)_{\mathbf{1}}\left(\nu+k+p_{\mathbf{K}}\right)_{\mathbf{1}}},
$$

and hence, letting $N \rightarrow \infty$,

$$
\begin{gathered}
\hat{b}_{\mathbf{K}}^{\nu}(\boldsymbol{\mu})=\prod_{k=0}^{\infty} \frac{\left(\nu+k+\ell_{\mathbf{K}}-\boldsymbol{\mu}\right)_{\mathbf{1}}\left(\nu+k+p_{\mathbf{K}}+\boldsymbol{\mu}^{*}\right)_{\mathbf{1}}}{\left(\nu+k+\ell_{\mathbf{K}}\right)_{\mathbf{1}}\left(\nu+k+p_{\mathbf{K}}\right)_{\mathbf{1}}} \\
=\prod_{k=0}^{\infty} \prod_{j=1}^{r_{\mathbf{K}}} \frac{\left(\nu+k+\ell_{\mathbf{K}}-\mu_{j}-\frac{a_{\mathbf{K}}}{2}(j-1)\right)\left(\nu+k+p_{\mathbf{K}}+\mu_{j}-\frac{a_{\mathbf{K}}}{2}\left(r_{\mathbf{K}}-j\right)\right)}{\left(\nu+k+\ell_{\mathbf{K}}-\frac{a_{\mathbf{K}}}{2}(j-1)\right)\left(\nu+k+p_{\mathbf{K}}-\frac{a_{\mathbf{K}}}{2}\left(r_{\mathbf{K}}-j\right)\right)}
\end{gathered}
$$




$$
\begin{gathered}
=\prod_{j=1}^{r_{\mathbf{K}}} \frac{\Gamma\left(\nu+\ell_{\mathbf{K}}-\frac{a_{\mathbf{K}}}{2}(j-1)\right) \Gamma\left(\nu+p_{\mathbf{K}}-\frac{a_{\mathbf{K}}}{2}\left(r_{\mathbf{K}}-j\right)\right)}{\Gamma\left(\nu+\ell_{\mathbf{K}}-\mu_{j}-\frac{a_{\mathbf{K}}}{2}(j-1)\right) \Gamma\left(\nu+p_{\mathbf{K}}+\mu_{j}-\frac{a_{\mathbf{K}}}{2}\left(r_{\mathbf{K}}-j\right)\right)} \\
=\frac{\Gamma_{\Omega_{\mathbf{K}}}\left(\nu+\ell_{\mathbf{K}}\right) \Gamma_{\Omega_{\mathbf{K}}}\left(\nu+p_{\mathbf{K}}\right)}{\Gamma_{\Omega_{\mathbf{K}}}\left(\nu+\ell_{\mathbf{K}}-\boldsymbol{\mu}\right) \Gamma_{\Omega_{\mathbf{K}}}\left(\nu+p_{\mathbf{K}}+\boldsymbol{\mu}^{*}\right)} .
\end{gathered}
$$

This completes the proof of Theorem 9.3 . In the complex case we obtain the formula Z2]

$$
\begin{aligned}
& \hat{b}_{\mathbf{C}}^{n}(\boldsymbol{m})=\prod_{j=1}^{r} \frac{\Gamma\left(n+\frac{p+1}{2}+\rho_{j}\right) \Gamma\left(n+\frac{p+1}{2}-\rho_{j}\right)}{\Gamma\left(n+\frac{p+1}{2}+\rho_{j}+m_{j}\right) \Gamma\left(n+\frac{p+1}{2}-\rho_{j}-m_{j}\right)} \\
& =\frac{\Gamma_{\Omega}\left(n+\ell_{\mathbf{C}}\right) \Gamma_{\Omega}(n+p)}{\Gamma_{\Omega}\left(n+\ell_{\mathbf{C}}-\boldsymbol{m}\right) \Gamma_{\Omega}\left(n+p+\boldsymbol{m}^{*}\right)}=(-1)^{\boldsymbol{m}} \frac{(-n)_{\boldsymbol{m}^{*}}}{(p+n)_{\boldsymbol{m}^{*}}}=\check{b}_{\mathbf{C}}^{-n}(\boldsymbol{\rho}+\boldsymbol{m}) .
\end{aligned}
$$

\section{Peter-Weyl expansions}

The main results of the paper concern the explicit Peter-Weyl decomposition of the asymptotic expansions for the Berezin transform and associated star operations (cf. AO and EU1, EU3, EU4 for previous work on this topic). Consider the real symmetric spaces $\dot{Z}_{\mathbf{K}}$ of rank $r_{\mathbf{K}}$. The results in [Z3] (for tube type domains) and [Z4] (for non-tube type domains) imply that for every signature $\boldsymbol{\mu}$ of length $r_{\mathbf{K}}$ there exists a unique $K^{\mathbf{K}}$-invariant polynomial $e_{\boldsymbol{\mu}}$ on $Z^{\mathbf{K}}$ satisfying

$$
e_{\boldsymbol{\mu}}(x)=E_{\boldsymbol{\mu}}^{+}(x, x)=E_{\boldsymbol{\mu}}^{+}(e, e) \Phi_{\boldsymbol{\mu}}^{+}\left(x^{2}\right)
$$

for all $x \in Z_{\mathbf{K}}^{+} \subset Z_{\mathbf{K}} \subset Z^{\mathbf{K}}$. Here $E_{\boldsymbol{\mu}}^{+}(x, x)$ is the Fischer-Fock reproducing kernel of type $\boldsymbol{\mu}$ for $Z_{\mathbf{K}}^{+}$and $\Phi_{\boldsymbol{\mu}}^{+}$is the spherical polynomial (normalized Jack polynomial) [FK]. As in (5.5) let $e_{\boldsymbol{\mu}}\left(\partial_{\mathbf{K}}\right)$ be the associated $K_{\mathbf{K}}$-invariant constant coefficient differential operator and define the corresponding $\dot{G}_{\mathbf{K}}$-invariant differential operator

$$
\left(e_{\boldsymbol{\mu}}\left(\dot{\partial}_{\mathbf{K}}\right) f\right)(x)=e_{\boldsymbol{\mu}}\left(\partial_{\mathbf{K}}\right)\left(f \circ \dot{\mathfrak{t}}_{x}\right)(0)
$$

on $\dot{Z}_{\mathbf{K}}$. The explicit and uniform formulas presented below hold for all cases except type (A). Type (A) seems to involve more than the basic Pochhammer symbols and will be excluded from consideration here.

Theorem 10.1. The Berezin transform has the Peter-Weyl asymptotic expansion

$$
\left(\dot{\beta}_{\mathbf{K}}^{\nu} f\right)(x) \equiv \sum_{\boldsymbol{\mu}} \frac{\left(e_{\boldsymbol{\mu}}\left(\dot{\partial}_{\mathbf{K}}\right) f\right)(x)}{[\nu]_{\boldsymbol{\mu}}}
$$

over all partitions $\boldsymbol{\mu}=\left(\mu_{1}, \ldots, \mu_{r_{\mathbf{K}}}\right)$, where

$$
[\nu]_{\boldsymbol{\mu}}:=\prod_{(i, j) \in \boldsymbol{\mu}}\left(\nu+\epsilon\left(i-\frac{1+c_{\mathbf{K}}}{2}-\frac{a_{\mathbf{K}}}{2}(j-1)\right)\right) .
$$

Specifically we have

$$
\begin{gathered}
\left(\beta_{\mathbf{K}}^{\nu} f\right)(x) \equiv \sum_{\boldsymbol{\mu}} \frac{\left(e_{\boldsymbol{\mu}}\left(\partial_{\mathbf{K}}\right) f\right)(x)}{\nu^{\boldsymbol{\mu}}}, \\
\left(\check{\beta}_{\mathbf{K}}^{\nu} f\right)(x) \equiv \sum_{\boldsymbol{\mu}} \frac{\left(e_{\boldsymbol{\mu}}\left(\check{\partial}_{\mathbf{K}}\right) f\right)(x)}{\left(\frac{1-c_{\mathbf{K}}}{2}+\nu\right)_{\boldsymbol{\mu}}} \\
\left(\hat{\beta}_{\mathbf{K}}^{\nu} f\right)(x) \equiv \sum_{\boldsymbol{\mu}} \frac{\left(e_{\boldsymbol{\mu}}\left(\hat{\partial}_{\mathbf{K}}\right) f\right)(x)}{(-1)^{\boldsymbol{\mu}}\left(\frac{1-c_{\mathbf{K}}}{2}-\nu\right)_{\boldsymbol{\mu}}} .
\end{gathered}
$$


Proof. The precise meaning of (10.2) is as follows: The $K_{\mathrm{K}^{-}}$-invariant real polynomials $p_{j}$ on $Z_{\mathbf{K}}$ occurring in the usual asymptotic expansion (5.6) of $\dot{\beta}_{\mathbf{K}}^{\nu}$ have a unique Peter-Weyl decomposition

$$
p_{j}=\sum_{\boldsymbol{\mu}} p_{j}^{\boldsymbol{\mu}} e_{\boldsymbol{\mu}}
$$

with real coefficients $p_{j}^{\mu}$. Then the assertion is

$$
\sum_{j=0}^{\infty} \frac{p_{j}^{\mu}}{\nu^{j}} \asymp \frac{1}{[\nu]_{\mu}}
$$

In fact, (10.4) implies

$$
\left(p_{j}\left(\dot{\partial}_{\mathbf{K}}\right) f\right)(x)=\sum_{\boldsymbol{\mu}} p_{j}^{\boldsymbol{\mu}}\left(e_{\boldsymbol{\mu}}\left(\dot{\partial}_{\mathbf{K}}\right) f\right)(x)
$$

and hence by (10.5)

$$
\dot{\beta}_{\mathbf{K}}^{\nu} \asymp \sum_{j=0}^{\infty} \frac{p_{j}\left(\dot{\partial}_{\mathbf{K}}\right)}{\nu^{j}}=\sum_{j=0}^{\infty} \frac{1}{\nu^{j}} \sum_{\boldsymbol{\mu}} p_{j}^{\boldsymbol{\mu}} e_{\boldsymbol{\mu}}\left(\dot{\partial}_{\mathbf{K}}\right)=\sum_{\boldsymbol{\mu}} e_{\boldsymbol{\mu}}\left(\dot{\partial}_{\mathbf{K}}\right) \sum_{j=0}^{\infty} \frac{p_{j}^{\boldsymbol{\mu}}}{\nu^{j}} \asymp \sum_{\boldsymbol{\mu}} \frac{e_{\boldsymbol{\mu}}\left(\dot{\partial}_{\mathbf{K}}\right)}{[\nu]_{\boldsymbol{\mu}}} .
$$

Using integral formulas contained in [Z3] and [Z4] an asymptotic expansion

$$
\frac{1}{\dot{c}_{\boldsymbol{\mu}}(\nu)} \asymp \sum_{j=0}^{\infty} \frac{p_{j}^{\boldsymbol{\mu}}}{\nu^{j}}
$$

is given in [EU4], with the following coefficients:

$$
\begin{gathered}
c_{\boldsymbol{\mu}}(\nu)=\nu^{|\boldsymbol{\mu}|}, \\
\check{c}_{\boldsymbol{\mu}}(\nu)=\left(\nu+\frac{\kappa d}{2 r_{\mathbf{K}}}+\ell_{\mathbf{K}}-p_{\mathbf{K}}\right)_{\boldsymbol{\mu}}=\left(\nu+\frac{1-c_{\mathbf{K}}}{2}\right)_{\boldsymbol{\mu}}, \\
\hat{c}_{\boldsymbol{\mu}}(\nu)^{-1}=\left(\nu+p_{\mathbf{K}}-\frac{\kappa d}{2 r_{\mathbf{K}}}\right)_{-\boldsymbol{\mu}^{*}}=\left(\nu+\ell_{\mathbf{K}}-\frac{1-c_{\mathbf{K}}}{2}\right)_{-\boldsymbol{\mu}^{*}} .
\end{gathered}
$$

Here $\boldsymbol{\mu}^{*}:=\left(\mu_{r_{\mathbf{K}}}, \ldots, \mu_{1}\right)$. To see that $\dot{c}_{\boldsymbol{\mu}}(\nu)=[\nu]_{\boldsymbol{\mu}}$ as defined above we note that for the compact type (3.16) implies

$$
\frac{1}{\left(-\nu+\frac{1-c_{\mathbf{K}}}{2}\right)_{\boldsymbol{\mu}}}=\frac{1}{\left(\frac{\kappa d}{2 r_{\mathbf{K}}}+\ell_{\mathbf{K}}-p_{\mathbf{K}}-\nu\right)_{\boldsymbol{\mu}}}=(-1)^{\boldsymbol{\mu}}\left(\nu+p_{\mathbf{K}}-\frac{\kappa d}{2 r_{\mathbf{K}}}\right)_{-\boldsymbol{\mu}^{*}} .
$$

Theorem 10.2. The inverse Berezin transform has the asymptotic Peter-Weyl expansion

$$
\left(\left(\dot{\beta}_{\mathbf{K}}^{\nu}\right)^{-1} f\right)(x) \equiv \sum_{\boldsymbol{\mu}} \frac{\left(e_{\boldsymbol{\mu}}\left(\dot{\partial}_{\mathbf{K}}\right) f\right)(x)}{\left[\epsilon p_{\mathbf{K}}-\nu\right]_{\boldsymbol{\mu}}}
$$

over all partitions $\boldsymbol{\mu}=\left(\mu_{1}, \ldots, \mu_{r_{\mathbf{K}}}\right)$, with coefficients $\left[\epsilon p_{\mathbf{K}}-\nu\right]_{\boldsymbol{\mu}}$ defined via (10.3). 
Specifically,

$$
\begin{gathered}
\left(\left(\beta_{\mathbf{K}}^{\nu}\right)^{-1} f\right)(x) \equiv \sum_{\boldsymbol{\mu}} \frac{\left(e_{\boldsymbol{\mu}}\left(\partial_{\mathbf{K}}\right) f\right)(x)}{(-\nu)^{\boldsymbol{\mu}}}, \\
\left(\left(\check{\beta}_{\mathbf{K}}^{\nu}\right)^{-1} f\right)(x) \equiv \sum_{\boldsymbol{\mu}} \frac{\left(e_{\boldsymbol{\mu}}\left(\check{\partial}_{\mathbf{K}}\right) f\right)(x)}{\left(\frac{1-c_{\mathbf{K}}}{2}+p_{\mathbf{K}}-\nu\right)_{\boldsymbol{\mu}}}, \\
\left(\left(\hat{\beta}_{\mathbf{K}}^{\nu}\right)^{-1} f\right)(x) \equiv \sum_{\boldsymbol{\mu}} \frac{\left(e_{\boldsymbol{\mu}}\left(\hat{\partial}_{\mathbf{K}}\right) f\right)(x)}{(-1)^{\boldsymbol{\mu}}\left(\frac{1-c_{\mathbf{K}}}{2}+p_{\mathbf{K}}+\nu\right)_{\boldsymbol{\mu}}} .
\end{gathered}
$$

Proof. We adopt the proof of Theorem 6 in [EU3] for the non-compact case. The main point is to show that the inverse Berezin transform has the asymptotic expansion

$$
\left(\dot{\beta}_{\mathbf{K}}^{\nu}\right)^{-1} \asymp \sum_{j=0}^{\infty} \frac{p_{j}\left(\dot{\partial}_{\mathbf{K}}\right)}{\left(\epsilon p_{\mathbf{K}}-\nu\right)^{j}}
$$

Define $\dot{\mathfrak{b}}_{\nu}(\boldsymbol{\mu})$ by

$$
\check{\mathfrak{b}}_{\nu}(\boldsymbol{\mu}):=\log \check{b}_{\nu}^{\mathbf{K}}(\boldsymbol{\rho}+\boldsymbol{\mu}), \quad \hat{\mathfrak{b}}_{\nu}(\boldsymbol{\mu}):=\log \hat{b}_{\nu}^{\mathbf{K}}(\boldsymbol{\mu}) .
$$

Since the Harish-Chandra map (between $\dot{G}_{\mathbf{K}}$-invariant operators and the associated spectral function) is an isomorphism, it is enough to show

$$
\dot{\mathfrak{b}}_{\epsilon p_{\mathbf{K}}-\nu}(\boldsymbol{\mu}) \asymp-\dot{\mathfrak{b}}_{\nu}(\boldsymbol{\mu}) .
$$

By $[\mathrm{BE}, \S 1.18(12)]$ there is an asymptotic expansion as $z \rightarrow+\infty$ :

$$
\log \Gamma\left(z+a+\frac{1}{2}\right) \asymp(z+a) \log z-z+\frac{\log (2 \pi)}{2}+\sum_{k=1}^{\infty} \frac{(-1)^{k+1} B_{k+1}\left(a+\frac{1}{2}\right)}{k(k+1)} z^{-k},
$$

where $B_{k}$ are the Bernoulli polynomials. In view of (9.2) and (9.4) we obtain the asymptotic expansion as $\nu \rightarrow+\infty$ :

$$
\dot{\mathfrak{b}}_{\nu}(\boldsymbol{\mu})=\epsilon \sum_{k=1}^{\infty} \frac{(-1)^{k+1}}{k(k+1)}\left(\nu-\frac{\epsilon p_{\mathbf{K}}}{2}\right)^{-k} \sum_{j=1}^{r}\left[B_{k+1}\left(\frac{1}{2} \pm\left(\rho_{j}+\mu_{j}\right)\right)-B_{k+1}\left(\frac{1}{2} \pm \rho_{j}\right)\right]
$$

As a consequence of the "reflection" property

$$
B_{k}(1-x)=(-1)^{k} B_{k}(x)
$$

of the Bernoulli polynomials, it follows that in (10.8) the terms with even $k$ vanish and only odd negative powers of $\nu-\frac{\epsilon p_{\mathbf{K}}}{2}$ occur. Since $-\left(\nu-\frac{\epsilon p_{\mathbf{K}}}{2}\right)=\left(\epsilon p_{\mathbf{K}}-\nu\right)-\frac{\epsilon p_{\mathbf{K}}}{2}$, the assertions (10.7) and hence (10.6) follow. The $K_{\mathbf{K}}$-invariant polynomials $q_{j}$ occurring in the usual asymptotic expansion (5.6) of $\left(\dot{\beta}_{\mathbf{K}}^{\nu}\right)^{-1}$ have a unique PeterWeyl decomposition

$$
q_{j}=\sum_{\boldsymbol{\mu}} q_{j}^{\boldsymbol{\mu}} e_{\boldsymbol{\mu}}
$$

with real coefficients $q_{j}^{\boldsymbol{\mu}}$. In view of (9.2) and (9.4) we have for each $\boldsymbol{\mu}$

$$
\sum_{j=0}^{\infty} \frac{q_{j}^{\boldsymbol{\mu}}}{\nu^{j}} \asymp \sum_{j=0}^{\infty} \frac{p_{j}^{\boldsymbol{\mu}}}{\left(\epsilon p_{\mathbf{K}}-\nu\right)^{j}}
$$


By (10.3) $[\nu]_{\mu}$ is a polynomial in $\nu$ which implies that the asymptotic expansion (10.5) also holds for $\nu \rightarrow-\infty$. Thus we may replace $\nu$ by $\epsilon p_{\mathbf{K}}-\nu$ and obtain

$$
\sum_{j=0}^{\infty} \frac{p_{j}^{\boldsymbol{\mu}}}{\left(\epsilon p_{\mathbf{K}}-\nu\right)^{j}} \asymp \frac{1}{\left[\epsilon p_{\mathbf{K}}-\nu\right]_{\boldsymbol{\mu}}} .
$$

Combining this with (10.11) it follows that

$$
\sum_{j=0}^{\infty} \frac{q_{j}^{\boldsymbol{\mu}}}{\nu^{j}} \asymp \frac{1}{\left[\epsilon p_{\mathbf{K}}-\nu\right]_{\boldsymbol{\mu}}} .
$$

Since (10.10) implies

$$
\left(q_{j}\left(\dot{\partial}_{\mathbf{K}}\right) f\right)(x)=\sum_{\boldsymbol{\mu}} q_{j}^{\boldsymbol{\mu}}\left(e_{\boldsymbol{\mu}}\left(\dot{\partial}_{\mathbf{K}}\right) f\right)(x)
$$

it follows from (10.13) that

$$
\begin{aligned}
\left(\dot{\beta}_{\mathbf{K}}^{\nu}\right)^{-1} \asymp \sum_{j=0}^{\infty} \frac{q_{j}\left(\dot{\partial}_{\mathbf{K}}\right)}{\nu^{j}}= & \sum_{j=0}^{\infty} \frac{1}{\nu^{j}} \sum_{\boldsymbol{\mu}} q_{j}^{\boldsymbol{\mu}} e_{\boldsymbol{\mu}}\left(\dot{\partial}_{\mathbf{K}}\right)=\sum_{\boldsymbol{\mu}} e_{\boldsymbol{\mu}}\left(\dot{\partial}_{\mathbf{K}}\right) \sum_{j=0}^{\infty} \frac{q_{j}^{\boldsymbol{\mu}}}{\nu^{j}} \\
& \asymp \sum_{\boldsymbol{\mu}} \frac{e_{\boldsymbol{\mu}}\left(\dot{\partial}_{\mathbf{K}}\right)}{\left[\epsilon p_{\mathbf{K}}-\nu\right]_{\boldsymbol{\mu}}} .
\end{aligned}
$$

Our first main result, in the general setting, gives the Peter-Weyl decomposition for the asymptotic expansion of the Berezin star-reduct. Consider the $\dot{G}_{\mathbf{K}}$-invariant "anti-holomorphic" differential operator $\overline{e_{\boldsymbol{\mu}}\left(\dot{\partial}^{\mathbf{K}}\right)}$ from $\dot{Z}^{\mathbf{K}}$ to $\dot{Z}_{\mathbf{K}}$ induced by the polynomials $e_{\boldsymbol{\mu}}$ defined in (10.1).

Theorem 10.3. The Berezin star-reduct has the asymptotic Peter-Weyl expansion as $\nu \rightarrow \infty$ :

$$
\left(\overline{\dot{*}}_{\nu}^{\mathbf{K}} F\right)(x) \equiv \sum_{\boldsymbol{\mu}} \frac{\left.\overline{\left(e_{\boldsymbol{\mu}}\left(\dot{\partial}^{\mathbf{K}}\right)\right.} F\right)(x)}{[\nu]_{\boldsymbol{\mu}}}
$$

for all $x \in \dot{Z}_{\mathbf{K}}$, with $[\nu]_{\boldsymbol{\mu}}$ as in (10.3). Specifically,

$$
\begin{gathered}
\left(\bar{*}_{\mathbf{K}}^{\nu} F\right)(x) \equiv \sum_{\boldsymbol{\mu}} \frac{\left(\overline{e_{\boldsymbol{\mu}}\left(\partial^{\mathbf{K}}\right)} F\right)(x)}{\nu^{\boldsymbol{\mu}}}, \\
\left(\overline{\widetilde{*}}_{\mathbf{K}}^{\nu} F\right)(x) \equiv \sum_{\boldsymbol{\mu}} \frac{\left(\overline{e_{\boldsymbol{\mu}}\left(\check{\partial}^{\mathbf{K}}\right)} F\right)(x)}{\left(\frac{1-c_{\mathbf{K}}}{2}+\nu\right)_{\boldsymbol{\mu}}}, \\
\left(\overline{\hat{*}}_{\mathbf{K}}^{\nu} F\right)(x) \equiv \sum_{\boldsymbol{\mu}} \frac{\left(\overline{e_{\boldsymbol{\mu}}\left(\hat{\partial}^{\mathbf{K}}\right)} F\right)(x)}{(-1)^{\boldsymbol{\mu}}\left(\frac{1-c_{\mathbf{K}}}{2}-\nu\right)_{\boldsymbol{\mu}}} .
\end{gathered}
$$

Proof. Since $p_{j}^{\boldsymbol{\mu}} \in \mathbf{R}$ the Peter-Weyl decomposition (10.4) of $p_{j}$ implies

$$
\left(\overline{p_{j}\left(\dot{\partial}^{\mathbf{K}}\right)} F\right)(x)=\sum_{\boldsymbol{\mu}} p_{j}^{\boldsymbol{\mu}}\left(\overline{e_{\boldsymbol{\mu}}\left(\dot{\partial}^{\mathbf{K}}\right)} F\right)(x) .
$$


In view of (10.5) this yields

$$
\dot{*}_{\nu}^{\mathbf{K}} \asymp \sum_{j=0}^{\infty} \frac{\overline{p_{j}\left(\dot{\partial}^{\mathbf{K}}\right)}}{\nu^{j}} \asymp \sum_{j=0}^{\infty} \frac{1}{\nu^{j}} \sum_{\boldsymbol{\mu}} p_{j}^{\boldsymbol{\mu}} \overline{e_{\boldsymbol{\mu}}\left(\dot{\partial}^{\mathbf{K}}\right)}=\sum_{\boldsymbol{\mu}} \overline{e_{\boldsymbol{\mu}}\left(\dot{\partial}^{\mathbf{K}}\right)} \sum_{j=0}^{\infty} \frac{p_{j}^{\boldsymbol{\mu}}}{\nu^{j}} \asymp \sum_{\boldsymbol{\mu}} \frac{\overline{e_{\boldsymbol{\mu}}\left(\dot{\partial}^{\mathbf{K}}\right)}}{[\nu]_{\boldsymbol{\mu}}} .
$$

We emphasize that this formal reduction to the Peter-Weyl decomposition of the Berezin transform rests on the holomorphic factorization property proved above.

Theorem 10.4. The Toeplitz star-reduct has the asymptotic Peter-Weyl expansion as $\nu \rightarrow \infty$ :

$$
\left(\dot{*}_{\nu}^{\mathbf{K}} F\right)(x) \equiv \sum_{\boldsymbol{\mu}} \frac{\left(e_{\boldsymbol{\mu}}\left(\dot{\partial}^{\mathbf{K}}\right) F\right)(x)}{\left[\epsilon p_{\mathbf{K}}-\nu\right]_{\boldsymbol{\mu}}}
$$

for all $x \in \dot{Z}_{\mathbf{K}}$, where $\left[\epsilon p_{\mathbf{K}}-\nu\right]_{\boldsymbol{\mu}}$ is defined via (10.3). Specifically,

$$
\begin{gathered}
\left(*_{\nu}^{\mathbf{K}} F\right)(x) \equiv \sum_{\boldsymbol{\mu}} \frac{\left(e_{\boldsymbol{\mu}}\left(\partial^{\mathbf{K}}\right) F\right)(x)}{(-\nu)^{\boldsymbol{\mu}}}, \\
\left(\check{*}_{\nu}^{\mathbf{K}} F\right)(x) \equiv \sum_{\boldsymbol{\mu}} \frac{\left(e_{\boldsymbol{\mu}}\left(\check{\partial}^{\mathbf{K}}\right) F\right)(x)}{\left(\frac{1-c_{\mathbf{K}}}{2}+p_{\mathbf{K}}-\nu\right)_{\boldsymbol{\mu}}}, \\
\left(\hat{*}_{\nu}^{\mathbf{K}} F\right)(x) \equiv \sum_{\boldsymbol{\mu}} \frac{\left(e_{\boldsymbol{\mu}}\left(\hat{\partial}^{\mathbf{K}}\right) F\right)(x)}{(-1)^{\boldsymbol{\mu}}\left(\frac{1-c_{\mathbf{K}}}{2}+p_{\mathbf{K}}+\nu\right)_{\boldsymbol{\mu}}} .
\end{gathered}
$$

Proof. The Peter-Weyl decomposition (10.10) of $q_{j}$ implies

$$
\left(q_{j}\left(\dot{\partial}^{\mathbf{K}}\right) F\right)(x)=\sum_{\boldsymbol{\mu}} q_{j}^{\boldsymbol{\mu}}\left(e_{\boldsymbol{\mu}}\left(\dot{\partial}^{\mathbf{K}}\right) F\right)(x) .
$$

In view of (10.13) this yields

$$
\dot{*}_{\nu}^{\mathbf{K}} \asymp \sum_{j=0}^{\infty} \frac{q_{j}\left(\dot{\partial}^{\mathbf{K}}\right)}{\nu^{j}} \asymp \sum_{j=0}^{\infty} \frac{1}{\nu^{j}} \sum_{\boldsymbol{\mu}} q_{j}^{\boldsymbol{\mu}} e_{\boldsymbol{\mu}}\left(\dot{\partial}^{\mathbf{K}}\right)=\sum_{\boldsymbol{\mu}} e_{\boldsymbol{\mu}}\left(\dot{\partial}^{\mathbf{K}}\right) \sum_{j=0}^{\infty} \frac{q_{j}^{\boldsymbol{\mu}}}{\nu^{j}} \asymp \sum_{\boldsymbol{\mu}} \frac{e_{\boldsymbol{\mu}}\left(\dot{\partial}^{\mathbf{K}}\right)}{[\nu]_{\boldsymbol{\mu}}} .
$$

We emphasize that this formal reduction to the Peter-Weyl decomposition of the inverse Berezin transform rests on the anti-holomorphic factorization property proved above.

In the complex case, where $\nu=n$, these results determine the asymptotic expansion of the Berezin operator transform. We reformulate the corresponding statements and also indicate direct proofs which may be of independent interest. We have $c_{\mathbf{C}}=1$ and $\boldsymbol{\mu}=\boldsymbol{m}$ is a partition of length $r_{\mathbf{C}}=r$. The complexified hermitian Jordan triple $Z \times \bar{Z}$ has the polynomial algebra

$$
\mathcal{P}(Z \times \bar{Z})=\mathcal{P}(Z) \otimes \overline{\mathcal{P}(Z)}=\sum_{\boldsymbol{m}, \boldsymbol{n}} \mathcal{P}_{\boldsymbol{m}}(Z) \otimes \overline{\mathcal{P}_{\boldsymbol{n}}(Z)}
$$

for arbitrary partitions $\boldsymbol{m}, \boldsymbol{n}$ of length $r$. Moreover, the $K$-invariant "real" polynomial is the Fischer-Fock kernel function (3.4) with associated differential operator

$$
E_{\boldsymbol{m}}\left(\partial_{\mathbf{C}}\right):=\sum_{i=1}^{d_{\boldsymbol{m}}} u_{\boldsymbol{m}}^{i}(\partial) \overline{u_{\boldsymbol{m}}^{i}(\partial)}
$$


As in (5.11) define the corresponding $\dot{G}$-invariant differential operator $E_{\boldsymbol{m}}\left(\dot{\partial}_{\mathbf{C}}\right)$ on $\dot{Z}$ by

$$
\left(E_{\boldsymbol{m}}\left(\dot{\partial}_{\mathbf{C}}\right) f\right)(z)=E_{\boldsymbol{m}}\left(\partial_{\mathbf{C}}\right)\left(f \circ \dot{\mathfrak{t}}_{z}\right)(0) .
$$

Specializing Theorem 10.1 yields

Theorem 10.5. The Berezin operator transform has an asymptotic Peter-Weyl expansion as $n \rightarrow+\infty$ :

$$
\dot{B}_{n} f(z) \equiv \sum_{\boldsymbol{m}} \frac{\left(E_{\boldsymbol{m}}\left(\dot{\partial}_{\mathbf{C}}\right) f\right)(z)}{[n]_{\boldsymbol{m}}}
$$

over all partitions $\boldsymbol{m}=\left(m_{1}, \ldots, m_{r}\right)$, where

$$
[n]_{\boldsymbol{m}}=\prod_{(i, j) \in \boldsymbol{m}}\left(n+\epsilon\left(i-1-\frac{a}{2}(j-1)\right)\right) .
$$

Specifically,

$$
\begin{aligned}
& B_{n} f(z) \equiv \sum_{\boldsymbol{m}} \frac{\left(E_{\boldsymbol{m}}\left(\partial_{\mathbf{C}}\right) f\right)(z)}{n^{\boldsymbol{m}}}, \\
& \check{B}_{n} f(z) \equiv \sum_{\boldsymbol{m}} \frac{\left(E_{\boldsymbol{m}}\left(\check{\partial}_{\mathbf{C}}\right) f\right)(z)}{(n)_{\boldsymbol{m}}}, \\
& \hat{B}_{n} f(z) \equiv \sum_{\boldsymbol{m}} \frac{\left(E_{\boldsymbol{m}}\left(\hat{\partial}_{\mathbf{C}}\right) f\right)(z)}{(-1)^{|\boldsymbol{m}|}(-n)_{\boldsymbol{m}}} .
\end{aligned}
$$

Proof. This result appears as Theorem 1.1 in [EU4]. For completeness, here we include a more direct proof in the compact case. We assert that for all $N \in \mathbf{N}$ and $z \in Z$ we have

$$
\left|\hat{B}_{n} f(z)-\sum_{|\boldsymbol{m}|<N} \frac{E_{\boldsymbol{m}}\left(\hat{\partial}_{\mathbf{C}}\right)\left(f \circ \hat{\mathfrak{t}}_{z}\right)(0)}{(-1)^{|\boldsymbol{m}|}(-n)_{\boldsymbol{m}}}\right|=O\left(n^{-N}\right) .
$$

By $\hat{G}$-invariance, it is enough to consider $z=0$. We thus need to prove that for any $N \in \mathbf{N}$

$$
\left|\hat{C}_{n} \int_{Z} f \hat{K}^{-n} d \hat{\lambda}-\sum_{|\boldsymbol{m}|<N} \frac{\left(E_{\boldsymbol{m}}\left(\partial_{\mathbf{C}}\right) f\right)(0)}{(-1)^{|\boldsymbol{m}|}(-n)_{\boldsymbol{m}}}\right|=O\left(n^{-N}\right) \quad \text { as } n \rightarrow+\infty .
$$

From (5.12) it is immediate that this certainly holds if $f$ vanishes at $z=0$ to a sufficiently high order (the maximum of $N$ and the total degrees of $P_{j}, 0 \leq j \leq N$, will do). Thus we may further assume that $f$ coincides with a polynomial in $(z, \bar{z})$ in some neighborhood of $z=0$. Finally, simple estimates show that for any sesquipolynomial $F$ in $(z, \bar{z})$ and $\delta>0$, the integral

$$
\hat{C}_{n} \int_{|z|>\delta} F(z) \hat{K}(z, z)^{-n} d \hat{\lambda}(z)
$$

(which exists as soon as $n$ is large enough) decays exponentially fast as $n \rightarrow+\infty$. Thus we may actually suppose that $f$ is a polynomial in $z$ and $\bar{z}$. We claim that in that case we even have the equality

$$
\hat{C}_{n} \int_{Z} f \hat{K}^{-n} d \hat{\lambda}=\sum_{\boldsymbol{m}} \frac{\left(E_{\boldsymbol{m}}\left(\partial_{\mathbf{C}}\right) f\right)(0)}{(-1)^{|\boldsymbol{m}|}(-n)_{\boldsymbol{m}}}
$$


as soon as $n$ is large enough for the integral on the left to exist. (Since $f$ is a polynomial in $(z, \bar{z})$, the sum on the right-hand side is always finite.) By linearity, it suffices to take $f=p_{\mathbf{j}} \bar{q}_{\mathbf{k}}$ with $p_{\mathbf{j}} \in \mathcal{P}_{\mathbf{j}}(Z), q_{\mathbf{k}} \in \mathcal{P}_{\mathbf{k}}(Z)$. Let $n \geq \max \left(j_{1}, k_{1}\right)$. By the Schur lemma, the sesquilinear form on $\mathcal{P}_{\mathbf{j}}(Z) \times \mathcal{P}_{\mathbf{k}}(Z)$,

$$
\left(p_{\mathbf{j}} \mid q_{\mathbf{k}}\right)_{n}=\hat{C}_{n} \int_{Z} p_{\mathbf{j}} \overline{q_{\mathbf{k}}} \hat{K}^{-n} d \hat{\lambda}
$$

vanishes identically if $\mathbf{j} \neq \mathbf{k}$ and is proportional to the Fischer-Fock inner product $\left(q_{\mathbf{k}} \mid p_{\mathbf{j}}\right)_{F}$ if $\mathbf{j}=\mathbf{k}$. Thus

$$
\hat{C}_{n} \int_{Z} p_{\mathbf{j}} \overline{q_{\mathbf{k}}} \hat{K}^{-n} d \hat{\lambda}=\delta_{\mathbf{j k}} \frac{\left(p_{\mathbf{j}} \mid q_{\mathbf{k}}\right)_{F}}{[n]_{\mathbf{k}}}
$$

for some proportionality constant $[n]_{\mathbf{k}}$ (independent of $p_{\mathbf{j}}, q_{\mathbf{k}}$ ). The inner product in $H_{n}^{2}(\hat{Z}) \subset \mathcal{P}(Z)$ is thus given by

$$
(p \mid q)_{n}=\sum_{\mathbf{k} \subset r \times n} \frac{\left(p_{\mathbf{k}} \mid q_{\mathbf{k}}\right)_{F}}{[n]_{\mathbf{k}}},
$$

implying that the reproducing kernel of $H_{n}^{2}(\hat{Z})$ has the form

$$
\Delta^{n}(z,-w)=\sum_{\mathbf{k} \subset r \times n}[n]_{\mathbf{k}} E_{\mathbf{k}}(z,-w) .
$$

Comparing this with the Faraut-Korányi formula (3.5) gives

$$
[n]_{\mathbf{k}}=(-1)^{|\mathbf{k}|}(-n)_{\mathbf{k}} .
$$

On the other hand,

$$
\begin{aligned}
E_{\boldsymbol{m}}\left(\partial_{\mathbf{C}}\right)\left(p_{\mathbf{j}} \bar{q}_{\mathbf{k}}\right)(0) & =\sum_{i=1}^{d_{\boldsymbol{m}}}\left(u_{\boldsymbol{m}}^{i}(\partial) p_{\mathbf{j}}\right)(0)\left(\overline{u_{\boldsymbol{m}}^{i}(\partial)} \bar{q}_{\mathbf{k}}\right)(0) \\
& =\sum_{i=1}^{d_{\boldsymbol{m}}}\left(p_{\mathbf{j}} \mid u_{\boldsymbol{m}}^{i}\right)_{F}\left(u_{\boldsymbol{m}}^{i} \mid q_{\mathbf{k}}\right)_{F}=\delta_{\boldsymbol{m}_{\mathbf{j}}} \delta_{\mathbf{k} \boldsymbol{m}}\left(p_{\mathbf{j}} \mid q_{\mathbf{k}}\right)_{F}
\end{aligned}
$$

so that

$$
\sum_{\boldsymbol{m}} \frac{E_{\boldsymbol{m}}\left(\partial_{\mathbf{C}}\right)\left(p_{\mathbf{j}} \bar{q}_{\mathbf{k}}\right)(0)}{[n]_{\boldsymbol{m}}}=\delta_{\mathbf{j k}} \frac{\left(p_{\mathbf{j}} \mid q_{\mathbf{k}}\right)_{F}}{[n]_{\mathbf{k}}} .
$$

Comparing with (10.16), the claim follows.

In the non-compact hermitian setting, the analogue of the last proposition is due to Arazy and Ørsted [AO]. Using the "reflection" argument as in Theorem 10.2 we obtain

Theorem 10.6. The inverse Berezin operator transform has the asymptotic expansion as $n \rightarrow+\infty$ :

$$
\dot{B}_{n}^{-1} f(z) \equiv \sum_{\boldsymbol{m}} \frac{\left(E_{\boldsymbol{m}}\left(\dot{\partial}_{\mathbf{C}}\right) f\right)(z)}{[\epsilon p-n]_{\boldsymbol{m}}}
$$

over all partitions $\boldsymbol{m}=\left(m_{1}, \ldots, m_{r}\right)$, where $[\epsilon p-n]_{\boldsymbol{m}}$ is defined via (10.15). Specifically,

$$
B_{n}^{-1} f(z) \equiv \sum_{\boldsymbol{m}} \frac{\left(E_{\boldsymbol{m}}\left(\partial_{\mathbf{C}}\right) f\right)(z)}{(-n)^{\boldsymbol{m}}}
$$




$$
\begin{aligned}
\check{B}_{n}^{-1} f(z) & \equiv \sum_{\boldsymbol{m}} \frac{\left(E_{\boldsymbol{m}}\left(\check{\partial}_{\mathbf{C}}\right) f\right)(z)}{(p-n)_{\boldsymbol{m}}}, \\
\hat{B}_{n}^{-1} f(z) & \equiv \sum_{\boldsymbol{m}} \frac{\left(E_{\boldsymbol{m}}\left(\hat{\partial}_{\mathbf{C}}\right) f\right)(z)}{(-1)^{\boldsymbol{m}}(p+n)_{\boldsymbol{m}}} .
\end{aligned}
$$

Proof. Restricting our attention to the compact case one has to show that the $\hat{G}$-invariant differential operators $E_{\boldsymbol{m}}\left(\hat{\partial}_{\mathbf{C}}\right)$ on $\hat{Z}$ satisfy

$$
\hat{B}_{n} \cdot \sum_{\boldsymbol{m}} \frac{E_{\boldsymbol{m}}\left(\hat{\partial}_{\mathbf{C}}\right)}{(-1)^{\boldsymbol{m}}(p+n)_{\boldsymbol{m}}}=\hat{B}_{n} \cdot \sum_{\boldsymbol{m}}\left(-n-\frac{d}{r}\right)_{-\boldsymbol{m}^{*}} E_{\boldsymbol{m}}\left(\hat{\partial}_{\mathbf{C}}\right)=I
$$

as the product of two formal power series in $\frac{1}{n}$ of $\hat{G}$-invariant differential operators on $\hat{Z}$. This follows in the same way as in the proof of Theorem 10.2 , i.e. using (10.14) and the property

$$
\hat{\mathfrak{b}}_{n}(\boldsymbol{m})=-\hat{\mathfrak{b}}_{-p-n}(\boldsymbol{m})
$$

which in turn is a consequence of (10.9).

The Peter-Weyl decomposition of the star-products looks as follows. The holomorphic differential operator associated with the Fischer-Fock kernel functions (3.4) is

$$
E_{\boldsymbol{m}}\left(\partial^{\mathbf{C}}\right)=\sum_{i=1}^{d_{\boldsymbol{m}}} u_{\boldsymbol{m}}^{i}(\partial) \otimes \overline{u_{\boldsymbol{m}}^{i}(\partial)}
$$

As in (8.8) one defines $\dot{G}$ invariant bi-differential operators $E_{\boldsymbol{m}}\left(\dot{\partial}^{\mathbf{C}}\right)$ by

$$
E_{\boldsymbol{m}}\left(\dot{\partial}^{\mathbf{C}}\right)\left(f_{1} \otimes f_{2}\right)(z)=E_{\boldsymbol{m}}\left(\partial^{\mathbf{C}}\right)\left[\left(f_{1} \circ \dot{\mathfrak{t}}_{z}\right) \otimes\left(f_{2} \circ \dot{\mathfrak{t}}_{z}\right)\right](0,0) .
$$

The "anti-holomorphic" type $\overline{E_{\boldsymbol{m}}\left(\dot{\partial}^{\mathbf{C}}\right)}$ is defined analogously.

Theorem 10.7. The Berezin star-product has the Peter-Weyl expansion

$$
\left(f_{1} \overline{\dot{*}} f_{2}\right)(z) \equiv \sum_{\boldsymbol{m}} \frac{\overline{E_{\boldsymbol{m}}\left(\dot{\partial}^{\mathbf{C}}\right)}\left(f_{1} \otimes f_{2}\right)(z, \bar{z})}{[n]_{\boldsymbol{m}}}
$$

over all partitions $\boldsymbol{m}=\left(m_{1}, \ldots, m_{r}\right)$, for $[n]_{\boldsymbol{m}}$ as in (10.15). Specifically,

$$
\begin{aligned}
& \left(f_{1} \bar{*} f_{2}\right)(z) \equiv \sum_{\boldsymbol{m}} \frac{\overline{E_{\boldsymbol{m}}\left(\partial^{\mathbf{C}}\right)}\left(f_{1} \otimes f_{2}\right)(z, \bar{z})}{n^{\boldsymbol{m}}}, \\
& \left(f_{1} \underset{n}{\check{*}} f_{2}\right)(z) \equiv \sum_{\boldsymbol{m}} \frac{\overline{E_{\boldsymbol{m}}(\check{\partial} \mathbf{C})}\left(f_{1} \otimes f_{2}\right)(z, \bar{z})}{(n)_{\boldsymbol{m}}}, \\
& \left(f_{1} \hat{\bar{*}} f_{2}\right)(z) \equiv \sum_{\boldsymbol{m}} \frac{\overline{E_{\boldsymbol{m}}(\hat{\partial} \mathbf{C})}\left(f_{1} \otimes f_{2}\right)(z, \bar{z})}{(-1)^{|\boldsymbol{m}|}(-n)_{\boldsymbol{m}}}=\sum_{\boldsymbol{m}}\left(n+p-\frac{d}{r}\right)_{-\boldsymbol{m}^{*}} \overline{E_{\boldsymbol{m}}\left(\hat{\partial}^{\mathbf{C}}\right)}\left(f_{1} \otimes f_{2}\right)(z, \bar{z}) \text {. }
\end{aligned}
$$

Theorem 10.8. The Toeplitz star-product has the Peter-Weyl expansion

$$
\left(f_{1_{n} \dot{*} f_{2}}\right)(z) \equiv \sum_{\boldsymbol{m}} \frac{E_{\boldsymbol{m}}\left(\dot{\partial}^{\mathbf{C}}\right)\left(f_{1} \otimes f_{2}\right)(z, \bar{z})}{[\epsilon p-n]_{\boldsymbol{m}}}
$$

over all partitions $\boldsymbol{m}=\left(m_{1}, \ldots, m_{r}\right)$, with $[\epsilon p-n]_{\boldsymbol{m}}$ given via (10.15). Specifically,

$$
\left(f_{1} f_{n} f_{2}\right)(z) \equiv \sum_{\boldsymbol{m}} \frac{E_{\boldsymbol{m}}\left(\partial^{\mathbf{C}}\right)\left(f_{1} \otimes f_{2}\right)(z, \bar{z})}{(-1)^{\boldsymbol{m}} n^{\boldsymbol{m}}},
$$




$$
\begin{aligned}
& \left(f_{1^{*}} f_{2}\right)(z) \equiv \sum_{\boldsymbol{m}} \frac{E_{\boldsymbol{m}}\left(\check{\partial}^{\mathbf{C}}\right)\left(f_{1} \otimes f_{2}\right)(z, \bar{z})}{(p-n)_{\boldsymbol{m}}}, \\
& \left(f_{1_{n} \hat{*} f_{2}}\right)(z) \equiv \sum_{\boldsymbol{m}} \frac{E_{\boldsymbol{m}}\left(\hat{\partial}^{\mathbf{C}}\right)\left(f_{1} \otimes f_{2}\right)(z, \bar{z})}{(-1)^{\boldsymbol{m}}(p+n)_{\boldsymbol{m}}} .
\end{aligned}
$$

Proof. In the non-compact setting, the analogue of this result was proved in EU3. In the flat case this is the "anti-Wick" calculus. In the compact case we give a selfcontained proof which illustrates the main ideas of our approach in the complex hermitian setting. By Theorem 10.5, there is an asymptotic expansion

$$
\hat{B}_{n}=\sum_{\boldsymbol{m}}\left(n+p-\frac{d}{r}\right)_{-\boldsymbol{m}^{*}} E_{\boldsymbol{m}}\left(\hat{\partial}_{\mathbf{C}}\right)=\sum_{\boldsymbol{m}} \frac{(-1)^{|\boldsymbol{m}|}}{(-n)_{\boldsymbol{m}}} E_{\boldsymbol{m}}\left(\hat{\partial}_{\mathbf{C}}\right),
$$

where $E_{\boldsymbol{m}}\left(\hat{\partial}_{\mathbf{C}}\right)$ is the unique $\hat{G}$-invariant differential operator on $\hat{Z}$ determined by

$$
\left(E_{\boldsymbol{m}}\left(\hat{\partial}_{\mathbf{C}}\right) f\right)(z):=E_{\boldsymbol{m}}\left(\partial_{\mathbf{C}}\right)\left(f \circ \hat{\mathfrak{t}}_{z}\right)(0)
$$

for all $f \in \mathcal{C}^{\infty}(\hat{Z})$. Now consider the bi-differential operators $\mathcal{L}_{j}, j \geq 0$, determined by (6.16). By $\hat{G}$-invariance, each $\mathcal{L}_{j}$ is uniquely determined by its action at the origin, which must be given by

$$
\mathcal{L}_{j}\left(f_{1} \otimes f_{2}\right)(0)=\left.\ell_{j}\left(\partial_{z}, \bar{\partial}_{z}, \partial_{w}, \bar{\partial}_{w}\right) f_{1}(z) f_{2}(w)\right|_{z=w=0}
$$

with some (uniquely determined) $K$-invariant polynomial $\ell_{j}(z, \bar{z}, w, \bar{w})$ of $z, w \in Z$. By Theorem $8.3, \bar{\partial}_{z}$ and $\partial_{w}$ are actually absent, i.e. $\ell_{j}=\ell_{j}(z, \bar{w})$ is a polynomial in $(z, \bar{w})$. It therefore has to be a linear combination of the Fischer-Fock kernels:

$$
\ell_{j}(z, \bar{w})=\sum_{\boldsymbol{m}} l_{j \boldsymbol{m}} E_{\boldsymbol{m}}(z, w)
$$

with some constants $l_{j m}$. Thus

$$
\mathcal{L}_{j}\left(f_{1} \otimes f_{2}\right)=\sum_{\boldsymbol{m}} l_{j \boldsymbol{m}} E_{\boldsymbol{m}}\left(\hat{\partial}^{\mathbf{C}}\right)\left(f_{1} \otimes f_{2}\right) .
$$

As in [EU3], we call (10.18) the Peter-Weyl decomposition of $\mathcal{L}_{j}$, and $l_{j \boldsymbol{m}} E_{\boldsymbol{m}}\left(\hat{\partial}^{\mathbf{C}}\right)$ its $\boldsymbol{m}$-th Peter-Weyl component. The formal power series in $\frac{1}{n}$,

$$
\sum_{j=0}^{\infty} n^{-j} l_{j \boldsymbol{m}} E_{\boldsymbol{m}}\left(\hat{\partial}^{\mathbf{C}}\right)\left(f_{1} \otimes f_{2}\right)
$$

is, in the obvious sense, the $\boldsymbol{m}$-th Peter-Weyl component of the Toeplitz starproduct

$$
\sum_{j=0}^{\infty} n^{-j} \mathcal{L}_{j}\left(f_{1} \otimes f_{2}\right)=f_{1} \hat{*} f_{2}
$$

studied by many authors [BMS, Sch, $\mathrm{AE}$. The explicit description of the PeterWeyl components (10.19) will be based on the asymptotic expansion

$$
\sum_{j=0}^{\infty} n^{-j} l_{j \boldsymbol{m}}=\left(-n-\frac{d}{r}\right)_{-\boldsymbol{m}^{*}}=\frac{(-1)^{|\boldsymbol{m}|}}{(n+p)_{\boldsymbol{m}}} .
$$

For the proof of (10.20) note that since the Peter-Weyl components of a $\hat{G}$-invariant bi-differential operator (or, hence, of a power series in $\frac{1}{n}$ whose coefficients are such 
operators) are uniquely determined, it is enough to show that for any $f_{1}, f_{2} \in \mathcal{C}^{\infty}(\hat{Z})$ we have the asymptotic expansion

$$
\sum_{j=0}^{\infty} n^{-j} \mathcal{L}_{j}\left(f_{1} \otimes \bar{f}_{2}\right)=\sum_{\boldsymbol{m}}\left(-n-\frac{d}{r}\right)_{-\boldsymbol{m}^{*}} E_{\boldsymbol{m}}\left(\hat{\partial}^{\mathbf{C}}\right)\left(f_{1} \otimes \bar{f}_{2}\right) .
$$

By Proposition $5.4 \hat{S}_{n} \hat{T}_{n}=\hat{B}_{n}$ is invertible as a formal power series in $\frac{1}{n}$ (since $P_{0}=I$ ), hence we may apply it to both sides. We are thus reduced to proving the asymptotic expansion

$$
\hat{S}_{n}\left(\left(\hat{T}_{n} f_{1}\right)\left(\hat{T}_{n} \bar{f}_{2}\right)\right)=\hat{B}_{n}\left(\sum_{\boldsymbol{m}}\left(-n-\frac{d}{r}\right)_{-\boldsymbol{m}^{*}} E_{\boldsymbol{m}}\left(\hat{\partial}^{\mathbf{C}}\right)\left(f_{1} \otimes \bar{f}_{2}\right)\right) .
$$

By $\hat{G}$-invariance, it is further enough to prove this only at the origin:

$$
\left(\left(\hat{T}_{n} f_{1}\right)\left(\hat{T}_{n} \bar{f}_{2}\right) \mathbf{1} \mid \mathbf{1}\right)=\hat{B}_{n}\left(\sum_{\boldsymbol{m}}\left(-n-\frac{d}{r}\right)_{-\boldsymbol{m}^{*}} E_{\boldsymbol{m}}\left(\hat{\partial}^{\mathbf{C}}\right)\left(f_{1} \otimes \bar{f}_{2}\right)\right)(0) .
$$

Finally, since both $E_{\boldsymbol{m}}\left(\hat{\partial}^{\mathbf{C}}\right)$ and $\mathcal{L}_{j}$ have the "anti-holomorphic factorization property", i.e. involve only holomorphic derivatives of $f_{1}$ and anti-holomorphic derivatives of $\bar{f}_{2}$, we need only consider functions $f_{1}, f_{2}$ which are holomorphic in some neighborhood of the origin. Hence we may assume that $f_{1}, f_{2}$ are holomorphic in a neighborhood $U$ - which we can take to be $K$-invariant - of $0 \in Z$, and (since both $\mathcal{L}_{j}$ and $E_{\boldsymbol{m}}\left(\hat{\partial}^{\mathbf{C}}\right)$ are bi-differential, hence local, operators) vanish outside $U$. We claim that under these assumptions, both sides of (10.21) are actually equal to $f_{1}(0) \overline{f_{2}(0)}$, up to an error term which decays faster than any negative power of $n$ as $n \rightarrow+\infty$ (and hence is negligible from the point of view of our asymptotic expansions in negative powers of $n$ ). Indeed, for the left-hand side, we have

$$
\begin{aligned}
\left(\left(\hat{T}_{n} f_{1}\right)\left(\hat{T}_{n} \bar{f}_{2}\right) \mathbf{1} \mid \mathbf{1}\right)= & \left(\hat{P}_{n} \bar{f}_{2} \mid \bar{f}_{1}\right) \\
= & \hat{C}_{n}^{2} \int_{U} \int_{U} \hat{K}(z, z)^{-n} d \hat{\lambda}(z) \hat{K}(w, w)^{-n} d \hat{\lambda}(w) \\
& \cdot f_{1}(z) \overline{f_{2}(w)} \Delta(z,-w)^{n} .
\end{aligned}
$$

Since the measure $\hat{C}_{n} \hat{K}(z, z)^{-n} d \hat{\lambda}(z)=: d \hat{M}_{n}(z)$ is $K$-invariant, replacing $z, w$ by $k z, l w$, with $k, l \in K$, and integrating over $K$ we see that the last integral equals

$$
\begin{gathered}
\hat{C}_{n}^{2} \int_{U} \int_{U} \hat{K}(z, z)^{-n} d \hat{\lambda}(z) \hat{K}(w, w)^{-n} d \hat{\lambda}(w) \int_{K} \int_{K} d k d l f_{1}(k z) \overline{f_{2}(l w)} \Delta(k z,-l w)^{n} \\
=f_{1}(0) \overline{f_{2}(0)} \cdot \hat{M}_{n}(U)^{2}
\end{gathered}
$$

by the mean value property of holomorphic functions and the fact that $\Delta(0,0)=1$. On the other hand, easy estimates show that

$$
\frac{1}{\hat{C}_{n}}\left(1-\hat{M}_{n}(U)\right)=\int_{Z \backslash U} \frac{d \lambda(z)}{\Delta(z,-z)^{n}}=O\left(\delta^{n}\right)
$$

whenever $\delta>\delta_{0}$, where $\delta_{0}=\sup _{z \in Z \backslash U} \Delta(z,-z)^{-1}<1$. It follows that $\hat{M}_{n}(U)=$ $1-\hat{C}_{n} O\left(\delta^{n}\right)=1-O\left(n^{-\infty}\right)$, proving the claim for the left-hand side. For the right-hand side, note that by (8.7) and the holomorphy of $f_{1}$ and $f_{2}$ on $U$,

$$
E_{\boldsymbol{m}}\left(\partial^{\mathbf{C}}\right)\left(f_{1} \otimes \bar{f}_{2}\right)(0)=E_{\boldsymbol{m}}\left(\partial_{\mathbf{C}}\right)\left(f_{1} \bar{f}_{2}\right)(0),
$$

so $E_{\boldsymbol{m}}\left(\hat{\partial}^{\mathbf{C}}\right)\left(f_{1} \otimes \bar{f}_{2}\right)=E_{\boldsymbol{m}}\left(\hat{\partial}_{\mathbf{C}}\right)\left(f_{1} \bar{f}_{2}\right)$ on $U$. In view of (10.17) we are done. 


\section{REFERENCES}

[AE] S. Twareque Ali and Miroslav Engliš, Quantization methods: a guide for physicists and analysts, Rev. Math. Phys. 17 (2005), no. 4, 391-490, DOI 10.1142/S0129055X05002376. MR:2151954(2006k:81209)

[AO] Jonathan Arazy and Bent Ørsted, Asymptotic expansions of Berezin transforms, Indiana Univ. Math. J. 49 (2000), no. 1, 7-30, DOI 10.1512/iumj.2000.49.1815. MR.1777039 (2001i:32033)

[BE] Arthur Erdélyi, Wilhelm Magnus, Fritz Oberhettinger, and Francesco G. Tricomi, Higher transcendental functions. Vols. I, II, McGraw-Hill Book Company, Inc., New YorkToronto-London, 1953. Based, in part, on notes left by Harry Bateman. MR.0058756 $(15,419 \mathrm{i})$

[BLU] David Borthwick, Andrzej Lesniewski, and Harald Upmeier, Nonperturbative deformation quantization of Cartan domains, J. Funct. Anal. 113 (1993), no. 1, 153-176, DOI 10.1006/jfan.1993.1050. MR1214901 (94d:47065)

[BMS] Martin Bordemann, Eckhard Meinrenken, and Martin Schlichenmaier, Toeplitz quantization of Kähler manifolds and $\operatorname{gl}(N), N \rightarrow \infty$ limits, Comm. Math. Phys. 165 (1994), no. 2, 281-296. MR1301849 (96f:58067)

[C] Benjamin Cahen, Berezin quantization for holomorphic discrete series representations: the non-scalar case, Beitr. Algebra Geom. 53 (2012), no. 2, 461-471, DOI 10.1007/s13366011-0066-2. MR 2971754

[E1] Miroslav Engliš, Weighted Bergman kernels and quantization, Comm. Math. Phys. 227 (2002), no. 2, 211-241, DOI 10.1007/s002200200634. MR.1903645 (2003f:32003)

[E2] Miroslav Engliš, Berezin-Toeplitz quantization on the Schwartz space of bounded symmetric domains, J. Lie Theory 15 (2005), no. 1, 27-50. MR2115226 (2006m:47050)

[EU1] Miroslav Engliš and Harald Upmeier, Toeplitz quantization and asymptotic expansions for real bounded symmetric domains, Math. Z. 268 (2011), no. 3-4, 931-967, DOI 10.1007/s00209-010-0702-9. MR2818737 (2012m:32024)

[EU2] Miroslav Engliš and Harald Upmeier, Toeplitz quantization and asymptotic expansions: geometric construction, SIGMA Symmetry Integrability Geom. Methods Appl. 5 (2009), Paper 021, 30, DOI 10.3842/SIGMA.2009.021. MR2481471 (2011b:32032)

[EU3] Miroslav Engliš and Harald Upmeier, Toeplitz quantization and asymptotic expansions: Peter-Weyl decomposition, Integral Equations Operator Theory 68 (2010), no. 3, 427449, DOI 10.1007/s00020-010-1808-5. MR2735445 (2012b:47072)

[EU4] Miroslav Engliš and Harald Upmeier, Real Berezin transform and asymptotic expansion for symmetric spaces of compact and non-compact type, Recent progress in operator theory and its applications, Oper. Theory Adv. Appl., vol. 220, Birkhäuser/Springer Basel AG, Basel, 2012, pp. 97-114, DOI 10.1007/978-3-0348-0346-5_6. MR2953873

[EZ] Miroslav Engliš and Genkai Zhang, Ramadanov conjecture and line bundles over compact Hermitian symmetric spaces, Math. Z. 264 (2010), no. 4, 901-912, DOI 10.1007/s00209009-0495-x. MR2593299(2011d:32032)

[FK] J. Faraut and A. Korányi, Function spaces and reproducing kernels on bounded symmetric domains, J. Funct. Anal. 88 (1990), no. 1, 64-89, DOI 10.1016/0022-1236(90)90119-6. MR 1033914 (90m:32049)

[FR] Frank Forelli, Measures whose Poisson integrals are pluriharmonic, Illinois J. Math. 18 (1974), 373-388. MR0342723(49 \#7468)

$[\mathrm{H}]$ Sigurdur Helgason, Groups and geometric analysis, Pure and Applied Mathematics, vol. 113, Academic Press Inc., Orlando, FL, 1984. Integral geometry, invariant differential operators, and spherical functions. MR754767 (86c:22017)

[KS] Alexander V. Karabegov and Martin Schlichenmaier, Identification of BerezinToeplitz deformation quantization, J. Reine Angew. Math. 540 (2001), 49-76, DOI 10.1515/crll.2001.086. MR.1868597 (2002h:53152)

[Li] Ewa Ligocka, On the Forelli-Rudin construction and weighted Bergman projections, Studia Math. 94 (1989), no. 3, 257-272. MR1019793 (90i:32034)

[L] O. Loos, Bounded Symmetric Domains and Jordan Pairs, University of California, Irvine, 1977.

[Sch] Martin Schlichenmaier, Deformation quantization of compact Kähler manifolds by Berezin-Toeplitz quantization, Conférence Moshé Flato 1999, Vol. II (Dijon), Math. 
Phys. Stud., vol. 22, Kluwer Acad. Publ., Dordrecht, 2000, pp. 289-306. MR.1805922 (2001k:53177)

[UU] A. Unterberger and H. Upmeier, The Berezin transform and invariant differential operators, Comm. Math. Phys. 164 (1994), no. 3, 563-597. MR.1291245 (96h:58170)

[Z1] G. Zhang, Berezin transform for real bounded symmetric domains, Trans. Amer. Math. Soc. 353 (2001), no. 9, 3769-3787. MR1837258(2002f:32044)

[Z2] Genkai Zhang, Berezin transform on compact Hermitian symmetric spaces, Manuscripta Math. 97 (1998), no. 3, 371-388, DOI 10.1007/s002290050109. MR.1654800(2000c:22013)

[Z3] Genkai Zhang, Branching coefficients of holomorphic representations and Segal-Bargmann transform, J. Funct. Anal. 195 (2002), no. 2, 306-349, DOI 10.1006/jfan.2002.3957. MR.1940358 (2004f:32026)

[Z4] Genkai Zhang, Degenerate principal series representations and their holomorphic extensions, Adv. Math. 223 (2010), no. 5, 1495-1520, DOI 10.1016/j.aim.2009.09.014. MR2592500(2011i:22013)

Mathematics Institute, Silesian University at Opava, Na RybníčKu 1, 74601 Opava, Czech Republic - And - Mathematics Institute, Žitná 25, 11567 Prague 1, Czech RePUBLIC

E-mail address: englis@math.cas.cz

Fachbereich Mathematik, Universität Marburg, D-35032 Marburg, Germany

E-mail address: upmeier@mathematik.uni-marburg.de 